\title{
Linearização e projetivização \\ de problemas variacionais: \\ duas aplicações
}

\author{
Diego Mano Otero
}

TESE APRESENTADA

$\mathrm{AO}$

Instituto De MatemáticA E EstatísticA

DA

UNIVERSIDADE DE SÃo PAUlo

PARA

OBTENÇÃO DO TÍTULO

$\mathrm{DE}$

DOUTOR EM CIÊNCIAS

Programa: Matemática

Orientador: Prof. Dr. Carlos Eduardo Durán Fernandez

Durante o desenvolvimento deste trabalho o autor recebeu auxílio financeiro da CAPES e CNPq

São Paulo, Setembro de 2015 


\section{Linearização e projetivização de problemas variacionais: duas aplicações}

Esta versão da tese contém as correções e alterações sugeridas pela Comissão Julgadora durante a defesa da versão original do trabalho, realizada em 11/08/2015. Uma cópia da versão original está disponível no Instituto de Matemática e Estatística da Universidade de São Paulo.

Comissão Julgadora:

- Prof. Dr. Carlos Eduardo Durán Fernández (orientador) - IME-USP

- Prof. Dr. Paolo Piccione - IME-USP

- Prof. Dr. Pedro Antonio Santoro Salomão - IME-USP

- Prof. Dr. Francesco Mercuri - IMECC-UNICAMP

- Prof. Dr. Henrique de Barros Correia Vitório - DMAT-UFPE 


\section{Agradecimentos}

Agradeço à toda minha família por estar comigo durante esta longa viagem. Em especial aos meus pais pelo apoio sentimental, ao meu tio Tadeu e minha vó Stella pelo incentivo inicial à matemática, e aos meus avós Carmen e Pepe por me ajudarem à aprender inglês.

Agradeço ao professor Durán por todo este período de ensinamento, desde a graduação até o doutorado. Não seria possível adquirir o conhecimento da beleza simples da matemática que possuo hoje se não tivesse sido guiado por ele neste longo percurso.

Agradeço aos meus amigos que estiveram comigo durante o doutorado, em especial ao Nélio, André e Clayton pelas companhias, conversas e risadas. Terei sempre boas lembranças destes momentos.

Agradeço ao Eiichiro Oda por me contar a melhor história do mundo, sempre dando ensinamentos valiosos para vida.

Agradeço à minha noiva Pierella por estar junto nos momentos bons e maus que decorreram o projeto. Por me acompanhar, incentivar, ajudar, e deixar nossos dias mais felizes, mostrando sempre o nosso futuro. Esta tese dedico à ela.

Agradeço todos os integrantes da banca examinadora pelas críticas, comentários, correções e incentivos.

Por fim, agradeço à CAPES e ao CNPq pelo apoio financeiro no decorrer do doutorado. 


\section{Resumo}

OTERO, D. M. Linearização e projetivização de problemas variacionais: duas aplicações. 2015. 68 f. Tese (Doutorado) - Instituto de Matemática e Estatística, Universidade de São Paulo, São Paulo, 2015.

Esta tese estuda a geometria de problemas variacionais através da linearização e projetivização das suas equações de Euler - Lagrange. O processo de linearização fornece a passagem das equações de Euler - Lagrange para as equações de Jacobi; a minimalidade (local) de extremais está determinada pelo conceito de ponto conjugado, que tem natureza projetiva. Propriedades de minimalidade local são transformadas em propriedades de auto-interseção de uma curva na variedade de Grassmann adequada. Desenvolvemos este processo em duas aplicações: 1) O estudo da minimalidade local de extremais de problemas variacionais de ordem superior. Neste caso, encontramos uma curva não degenerada de planos isotrópicos num espaço vetorial simplético, que, após prolongamento por derivadas, fornece uma curva degenerada de planos Lagrangeanos cujas auto-interseções determinam a minimalidade. 2) No caso mais clássico de problemas de ordem um, estudamos a versão linear projetiva do problema inverso: dada uma equação diferencial de ordem dois, quando ela é a equação de Euler - Lagrange de um problema variacional? Veremos que as condições do problema inverso linear - projetivo fornecem informações sobre os possíveis Lagrangeanos, por exemplo a assinatura.

Palavras-chave: cálculo das variações, geometria simplética, curvas espalhantes. 


\section{Abstract}

OTERO, D. M. Linearization and projectivization of variational problems: two applications. 2015. 68 f. Tese (Doutorado) - Instituto de Matemática e Estatística, Universidade de São Paulo, São Paulo, 2010.

In this work we study the geometry of high order calculus of variations through the linearization and projectivization of their Euler-Lagrange equations. The linearization process provides the passage from the Euler-Lagrange equations to the Jacobi equations; the (local) minimality properties of the extremal is determined by conjugate points, which is a projective concept. Minimaltiy properties of the extremals are transformed into self-intersection propertie of curves in the appropriate Grassmann manifold. We develop this process in two instances: 1) The study of minimality properties of extremals of higher-order variational problems. In this case, we find a non-degenerate curve of isotropic subspaces, that, after prolongation by derivatives, gives a degenerate curve of Lagrangian planes whose self-intersections determine minimality. 2) In the classical case of order one variational problems, we study a projective-linear version of the inverse problem: given a second order differential equation, when is it the Euler-Lagrange equation of a variational problem? We will see that the conditions given by the linear-projective inverse problem provides information about the possible Lagrangians, for example, its signature.

Keywords: calculus of variations, symplectic geometry, fanning curves. 


\section{Sumário}

1 Introdução e Preliminares $\quad 1$

1.1 A Hessiana de Um Problema de Ordem Superior . . . . . . . . . . . . . . . . 3

1.2 Curvas na Grassmanniana Metade e Divisível . . . . . . . . . . . . . . . . . . . . 4

2 Geometria Simplética Projetiva para Funcionais Quadráticos:

Caso Unidimensional $\quad 9$

2.1 Identidade de Easwaran . . . . . . . . . . . . . . . . . . . . . 11

2.2 Curvas de Jacobi . . . . . . . . . . . . . . . . . . . . . . . . . 13

3 Sistemas Hamiltonianos Lineares $\quad 15$

3.1 Soluções Isotrópicas . . . . . . . . . . . . . . . . . . . . 15

3.2 Pontos Conjugados . . . . . . . . . . . . . . . . . . . . 17

3.3 Funcional Quadrático Hamiltoniano . . . . . . . . . . . . . . . . . . 18

4 Minimalidade e Curva de Isotrópicos $\quad 19$

4.1 Identidade de Picone . . . . . . . . . . . . . . . . . . . . . . . . . 20

4.2 Identidade de Picone na Reta . . . . . . . . . . . . . . . . . . 20

4.3 Da Equação de Jacobi para Sistemas Hamiltonianos . . . . . . . . . . . . . . . . 23

4.4 Transformação de Legendre . . . . . . . . . . . . . . . . . . . . . 25

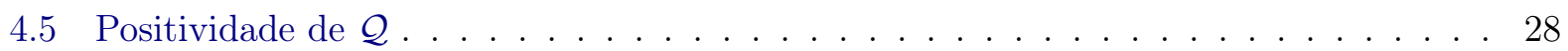

4.6 Curvas de Jacobi . . . . . . . . . . . . . . . . . . . . . . . 30

4.7 Prolongamentos Simpléticos _ . . . . . . . . . . . . . . . . . . . 30

4.8 Casos Particulares . . . . . . . . . . . . . . . . . . . . . . . 32

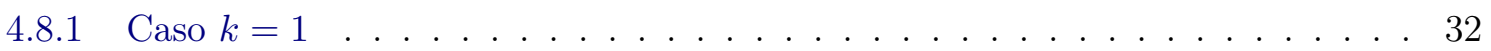

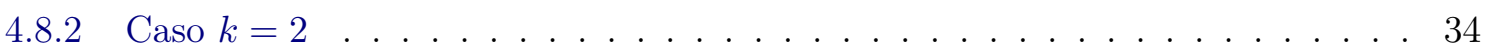

5 Problema Inverso Linear $\quad 37$

5.1 Condições de Helmholtz . . . . . . . . . . . . . . . . . . . . . . . . . . 37

5.2 Referenciais Normais e Schwarziana . . . . . . . . . . . . . . . . . . . 37

5.3 Wronskiano e Curva de Lagrangeanos . . . . . . . . . . . . . . . . . . . . . . 41

5.4 Condição de Comutatividade Generalizada . . . . . . . . . . . . . . . . . . . . 44

$\begin{array}{ll}\text { A Problemas Variacionais de Ordem Superior } & 51\end{array}$

A.1 Problemas Variacionais de Primeira Ordem . . . . . . . . . . . . . . . 51

A.2 Geometria dos Espaços Tangentes de Ordem Superior . . . . . . . . . . . . . 55

A.2.1 Problema Variacional de Ordem Superior . . . . . . . . . . . . . . 56 
A.2.2 Equações Diferenciais e Estrutura Quase Tangente . . . . . . . . . . . . . 57

A.2.3 Derivações Verticais . . . . . . . . . . . . . . . . . . . 61

A.2.4 Derivação de Tulczyjew . . . . . . . . . . . . . . . . . . . 62

A.2.5 Formalismo de Klein . . . . . . . . . . . . . . . . . . . . . 62

A.2.6 Transformação de Legendre . . . . . . . . . . . . . . . . . . . . 63

Referências Bibliográficas 


\section{Capítulo 1}

\section{Introdução e Preliminares}

Um tema de estudo no cálculo das variações consiste em aplicar sequencialmente operações, que chamaremos, de linearização e projetivização de um problema variacional. A linearização fornece a passagem das equações de Euler - Lagrange para as equações de Jacobi. Ponto conjugado da equação de Jacobi é um conceito projetivo; por exemplo, no caso Riemanniano se $Y$ é um campo de Jacobi com condições iniciais $Y(0)=0, \nabla_{t} Y(0)=V_{0}$, e tem um ponto conjugado em $t=t_{0}$, então obviamente $\lambda Y, \lambda \neq 0$, também possui um ponto conjugado em $t=t_{0}$.

Podemos considerar simultaneamente todos os campos de Jacobi "verticais" que satisfazem $Y(0)=0$, e estudar a evolução do plano gerado por eles. O que é equivalente a estudar a evolução do tangente as fibras verticais no duplo tangente sob o fluxo geodésico. Este processo leva naturalmente a teoria do índice de Maslov, onde se relaciona a topologia de intersecção de curvas da variedade Lagrangeana Grassmanniana com o trem estratificado de planos não transversais a um plano dado; para uma boa descrição temos estas duas referencias [GMPT99, MPT02]. Nos artigos [ÁPD09, ABR13], vemos além da topologia destas curvas, como podemos estudar a geometria de Cartan - Klein, e recuperamos a curvatura (como o operador de curvatura $X \mapsto R((X, \dot{\gamma}) \dot{\gamma})$. A abordagem desta relação pode ser feita através da linearização do fluxo geodésico, considerando curvas de Jacobi definidas por

$$
\ell_{(p, v)}(t)=D \Phi_{-t}\left(\Delta\left(\Phi_{t}(p, v)\right)\right),
$$

onde $\Delta\left(\Phi_{t}(p, v)\right)=\mathcal{V}_{\Phi_{t}(p, v)}(T M)$ é a distribuição em $T M$ dos campos verticais. Note que o espaço dos campos de Jacobi ao longo de $\gamma$ que satisfazem $J(0)=0$ pode ser identificado com o espaço vertical $\mathcal{V}_{(p, v)}(T M)=T_{v}\left(T_{p} M\right)$.

A curva $\ell_{(p, v)}$ será uma curva na Grassmanniana metade $\operatorname{Gr}\left(n, T_{(p, v)}(T M)\right)$, e, por causa do fluxo geodésico ser Hamiltoniano e a condição inicial ser Lagrangeana, estará na verdade contida na Lagrangiana Grassmanniana $\Lambda_{n}$. Este ponto de vista permite estudar de maneira muito mais "limpa" não somente variedades Riemannianas (por exemplo, variedades sem pontos conjugados como em [Pat99, Rug07]), mas também outras estruturas geométricas tais como variedades semiRiemannianas ([MPT02]), sub-Riemannianas ([ABR13]) e Finsler ([JV14]).

Tendo em vista este desenvolvimento e as relações naturais do cálculo variacional com geometria simplética, que consiste no caso particular da análise de Lagrangeanos dependendo de derivações até primeira ordem, o objetivo inicial do projeto foi estender esta abordagem a problemas variacionais de ordem superior.

Para ilustrar, considere o caso de problemas variacionais de ordem $k=2$ (onde a equação de Euler-Lagrange terá ordem $4=2 \times 2$ ). A notação e a teoria a seguir está contida no apêndice A. Considere uma equação de ordem $4=2 \times 2 \mathrm{em}$ uma variedade $Q$, isto é, um campo $\xi: T^{3} Q \rightarrow$ $T\left(T^{3} Q\right)$ que satisfaz $J_{1} \xi=C_{1}$, onde $J_{1}$ é a estrutura quase-tangente canônica de ordem superior e 
$C_{1}$ é o campo de Liouville de $T^{3} Q$, que localmente são dados por

$$
J_{1}=\sum_{r=0}^{2} \frac{\partial}{\partial q_{r+1}^{A}} \otimes d q_{r}^{A} \quad, \quad C_{1}=\sum_{r=1}^{3} q_{r}^{A} \frac{\partial}{\partial q_{r}^{A}},
$$

onde usamos a convenção de somatória no índice $A, 1 \leq A \leq n=\operatorname{dim} Q$ (usaremos esta convenção ao longo do texto quando for necessário). Com isso $\xi$ localmente será dado por

$$
\xi=\sum_{r=0}^{2} q_{(r+1)}^{A} \frac{\partial}{\partial q_{r}^{A}}+\xi^{A} \frac{\partial}{\partial q_{3}^{A}}
$$

onde $\xi^{A}: T^{3} Q \rightarrow \mathbb{R}$. Assim toda curva $q: \mathbb{R} \rightarrow Q$ suave cuja levantada para $T^{3} Q$ seja integral do campo $\xi$, terá que satisfazer localmente

$$
\frac{d^{4} q^{A}}{d t^{4}}=\xi^{A}\left(q(t), \frac{d q}{d t}, \frac{d^{2} q}{d t^{2}}, \frac{d^{3} q}{d t^{3}}\right)
$$

que é uma equação de ordem 4.

Sendo $\phi_{t}$ o fluxo de $\xi$, a linearização da equação (1.1) será dada pela diferencial do fluxo. Fixando um ponto $j^{3} c(0) \in T^{3} Q$ e $X \in T_{j^{3} c(0)}\left(T^{3} Q\right)$, consideramos a seguinte curva $t \mapsto X_{v}(t)=d \phi_{t}(X)$ que será um campo de vetores ao longo de $\gamma(t)=\phi_{t}\left(j^{3} c(0)\right)$.

Usando as coordenadas naturais de $T\left(T^{3} Q\right)$ podemos escrever

$$
X_{v}(t)=\left.a^{A}(t) \frac{\partial}{\partial q_{0}^{A}}\right|_{\phi_{t}\left(j^{3} c(0)\right)}+\left.b^{A}(t) \frac{\partial}{\partial q_{1}^{A}}\right|_{\phi_{t}\left(j^{3} c(0)\right)}+\left.c^{A}(t) \frac{\partial}{\partial q_{2}^{A}}\right|_{\phi_{t}\left(j^{3} c(0)\right)}+\left.d^{A}(t) \frac{\partial}{\partial q_{3}^{A}}\right|_{\phi_{t}\left(j^{3} c(0)\right)} .
$$

Observando que $\mathcal{L}_{\xi} X_{v}(t)=0$ teremos que as coordenadas $a^{A}, b^{A}, c^{A}, d^{A}$ acima terão que satisfazer o seguinte sistema diferencial linear

$$
\left(\begin{array}{c}
\dot{a^{A}} \\
\dot{b^{A}} \\
\dot{c^{A}} \\
\dot{d^{A}}
\end{array}\right)=\left(\begin{array}{cccc}
0 & \mathrm{Id} & 0 & 0 \\
0 & 0 & \mathrm{Id} & 0 \\
0 & 0 & 0 & \mathrm{Id} \\
\frac{\partial \xi^{B}}{\partial q_{0}^{A}} & \frac{\partial \xi^{B}}{\partial q_{1}^{A}} & \frac{\partial \xi^{B}}{\partial q_{2}^{A}} & \frac{\partial \xi^{B}}{\partial q_{3}^{A}}
\end{array}\right)\left(\begin{array}{c}
a^{A} \\
b^{A} \\
c^{A} \\
d^{A}
\end{array}\right)
$$

O sistema (1.2) representa o fluxo linearizado.

Agora, considerando um Lagrangeano $L: T^{2} Q \rightarrow \mathbb{R}$, o campo de Euler-Lagrange $\xi=\xi_{L}$ pode ser construído de tal maneira que a equação (1.1) será a equação de Euler-Lagrange com relação à $L$. Como o sistema (1.2) é a linearização de (1.1), o sistema (1.2) será equivalente à equação de Jacobi relacionada à $L$. O que acabamos de descrever seria o processo de linearização do problema variacional.

O processo de projetivização, neste caso, consiste em tomar $4 n$ soluções li.i., $h_{i}=\left(a_{i}^{A}\right), i=$ $1, \ldots, 4 n$ e construir um referencial matricial cujas linhas sejam estas soluções:

$$
\mathcal{A}=\left(\begin{array}{c}
h_{1}^{T} \\
\vdots \\
h_{4 n}^{T}
\end{array}\right)
$$

O referencial $\mathcal{A}$ é uma matriz de ordem $4 n \times n$ e, supondo algumas hipóteses sobre as condições iniciais, teremos que a curva de subespaços $\ell:[a, b] \rightarrow \operatorname{Gr}(n, 4 n)$, dada pela combinação linear das colunas de $\mathcal{A}$ em cada instante, será espalhante no sentido de [dAP10].

Teremos ainda que $\ell$ será um curva de subespaços isotrópicos com relação à forma simplética canônica $\omega_{\text {can }}$ em $\mathbb{R}^{4 n}$ de forma que as prolongações $j^{1} \ell$ e $j^{2} \ell$ serão curvas de subespaços Lagrangeanos e coisotrópicos, respectivamente. 
Essa curva é importante pois, como demonstramos no texto, ela dará a condição de minimalidade para o problema variacional do Lagrangeano $L$. Com um pouco mais de detalhes, se tivermos que $j^{1} \ell(0) \cap j^{1}(t)=\{0\}$ para todo $t \in(a, b]$ e a condição de Legendre estrita $\left(\frac{\partial L}{\partial q_{2}^{A} \partial q_{2}^{B}}>0\right)$, então a curva $\phi_{t}\left(j^{3} c(0)\right)$ será um mínimo do funcional

$$
c \mapsto \mathcal{F}(c)=\int_{a}^{b} L\left(j^{2} c(t)\right) d t .
$$

A questão da identificação de problemas variacionais (problema inverso do cálculo das variações) é interessante, e pode ser colocado da seguinte forma: dada uma equação de segunda ordem, quando que tal problema provém de um problema variacional, isto é, quando que a equação é uma equação de Euler-Lagrange de um Lagrangeano?

No contexto da linearização e projetivização chegamos na resposta do problema em termos de curvas espalhantes na Grassmanniana metade $\ell: I \rightarrow \operatorname{Gr}(n ; 2 n)$. Dizemos que tal curva $\ell$ é variacional (Jacobi) quando para algum referencial $\mathcal{A}$ de $\ell$ a equação diferencial associada à $\mathcal{A}$ vem de um problema variacional. E conseguimos o seguinte resultado: sendo $\ell$ uma curva espalhante, então $\ell$ é variacional se, e somente se, existir uma forma simplética $\omega$ tal que $\ell$ é uma curva de Lagrangeanos para $\omega$. Através dos invariantes de [ÁPD09] seremos capazes de distinguir quando uma curva espalhante na Grassmanniana vem de um problema variacional.

A organização do trabalho é: logo a seguir descreveremos as preliminares básicas para poder entender os cálculos dos invariantes de curvas espalhantes na Grassmanniana metade. Um ponto importante para salientar é que, depois de saber que o Hessiano de um problema variacional está bem definido, o restante dos cálculos pode ser feito em $\mathbb{R}^{N}$ via, por exemplo, referenciais. Assim, o núcleo da tese está escrito em um $\mathbb{R}^{N}$ adequado fixo, e citaremos o apêndice A para as considerações da linguagem topológico-diferencial. Nos capítulos 2, 3 e 4 desenvolvemos e estudamos o análogo da curva de Jacobi para problemas de ordem superior. De maneira mais concreta, no capítulo 2 descrevemos o problema unidimensional, cuja solução é baseada em identidades clássicas que aparecem no trabalho de Easwaran ([Eas76]). Isto é feito como introdução pois trata-se de um caso combinatoriamente mais simples. Para dimensão geral conseguimos fazer os cálculos usando a transformação de Legendre apoiados na versão Hamiltoniana encontrada no trabalho de Coppel ([Cop71]). Resumimos o trabalho de Coppel no capítulo 3 para logo após, no capítulo 4, definirmos a curva de Jacobi que dará a condição de minimalidade do funcional, e este é o resultado principal da tese. No capítulo 5 voltamos ao caso padrão de Lagrangeanos de ordem um, e vimos como os invariantes das curvas de Jacobi fornecem informação sobre o problema inverso do cálculo das variações.

\subsection{A Hessiana de Um Problema de Ordem Superior}

O problema do cálculo das variações de ordem superior em uma variedade $Q$ consiste em descobrir quais são os pontos críticos do seguinte funcional

$$
\mathcal{F}(c)=\int_{a}^{b} L\left(j^{k} c(t)\right) d t
$$

onde $L: T^{k} Q \rightarrow \mathbb{R}$ é chamado de Lagrangeano de ordem $k, c$ é uma curva em $Q$ definida no intervalo $[a, b]$ e $j^{k} c$ é o prolongamento ao espaço tangente de ordem $k$. Para mais detalhes de como é construído tal prolongamento, e outras notações que usaremos neste capítulo e nos demais, como as coordenadas locais naturais de $T^{k} Q$, consulte o apêndice A.

Vamos impor ainda que o funcional $\mathcal{F}$ acima está definido no espaço de curvas com extremos fixos $j^{k-1} q_{a}, j^{k-1} q_{b} \in T^{k-1} Q$, e vamos denotar tal espaço por $\Omega_{q_{a}, q_{b}}[a, b]$. Simbolicamente

$$
\Omega_{q_{a}, q_{b}}[a, b]=\left\{c:[a, b] \rightarrow Q \mid j^{k-1} c(a)=j^{k-1} q_{a} \text { e } j^{k-1} c(b)=j^{k-1} q_{b}\right\} .
$$


Falando de outro modo, $\Omega_{q_{a}, q_{b}}[a, b]$ é o espaço de todas as curvas $c$ em $Q$ tais que as $k-1$ derivadas no extremo $a$ e no extremo $b$ são iguais à valores pré fixados.

Um ponto crítico $\gamma$ do funcional acima pode ser encontrado usando diferentes abordagens dentre elas citamos Gelfand-Fomin ([GF00]), que usa coordenadas locais e chega à uma equação que $\gamma$ deve satisfazer, e a abordagem de Léon-Rodrigues ([dLR85]) que usa a regularidade do Lagrangeano $L$ para desenvolver a geometria simplética de tal problema (mais detalhes no apêndice A).

O ponto mais importante é que para discutirmos a minimalidade de tal ponto crítico $\gamma$ precisamos analisar a Hessiana do funcional $\mathcal{F}$. Seguindo o mesmo raciocínio que [Mil73, Pal63, Pal69, Pal68], a Hessiana de $\mathcal{F}$ no ponto crítico $\gamma$ está bem definida e ela será um funcional quadrático definido no espaço tangente $T_{\gamma} \Omega_{q_{a}, q_{b}}[a, b]$. Tal espaço corresponde ao espaço de campos de vetores ao longo de $\gamma$ cujas $k-1$ derivadas nos extremos $a$ e $b$ se anulam. Assim, como a Hessiana está bem definida, escolhemos um referencial de campos de vetores ao longo de $\gamma, v_{1}(t), \ldots, v_{n}(t)$ que geram $T_{\gamma(t)} Q$ para cada $t$. Em termos deste referencial cada elemento $X \in T_{\gamma} \Omega_{q_{a}, q_{b}}[a, b]$ pode ser escrito como $X(t)=\sum_{i} h_{i}(t) v_{i}(t)$, e assim podemos identificar $T_{\gamma} \Omega_{q_{a}, q_{b}}[a, b]$ com o seguinte espaço de curvas em $\mathbb{R}^{n}$

$$
C_{k}^{\infty}\left([a, b], \mathbb{R}^{n}\right)=\left\{h \in C^{\infty}\left([a, b], \mathbb{R}^{n}\right) \mid h^{(i)}(a)=h^{(i)}(b)=0, \forall i=0,1, \ldots k-1\right\} .
$$

Portanto podemos trabalhar em coordenadas locais e chegamos na seguinte expressão da Hessiana de $\mathcal{F}$ no ponto crítico $\gamma$

$$
\mathcal{Q}(h)=\int_{a}^{b} \sum_{i, j=0}^{k} h^{(i)}{ }^{T} L_{i j}(t) h^{(j)} d t,
$$

onde cada $L_{i j}$ é uma curva suave de matrizes $n \times n$ que depende do Lagrangeano $L$ e de suas derivadas até segunda ordem. Um ponto importante nesta conta é que a matriz $L_{k k}$, que acompanha as derivadas de maior ordem na expressão de $\mathcal{Q}$, vai corresponder ao Hessiano do Lagrangeano $L$ com relação as $k$-velocidades. Se os referenciais acima forem construídos através de coordenadas naturais teremos

$$
L_{k k}=\left(\frac{\partial^{2} L}{\partial q_{(k)}^{A} \partial q_{(k)}^{B}}\left(j^{k} \gamma(t)\right)\right) .
$$

Observe que $L_{k k}>0$ será uma condição que não irá depender do referencial acima escolhido.

Um dos objetivos deste trabalho, que será desenvolvido nos próximos capítulos, será encontrar condições necessárias que garantam a positividade do funcional $\mathcal{Q}$ acima. O resultado que obtemos é que tais condições serão dadas em termos da positividade de $L_{k k}$ e da não intersecção do prolongamento de uma curva de subespaços isotrópicos (chamada de curva de Jacobi) com um certo subespaço.

É importante observar, como é feito em [GF00], que a positividade de $\mathcal{Q}$ irá garantir a positividade forte do funcional $\mathcal{Q}$, isto é, teremos que $\mathcal{Q}(h) \geq C\|h\|$ para alguma constante $C$ e para uma norma apropriada no espaço de curvas em $\mathbb{R}^{n}$ que são $k$ vezes diferenciáveis. O funcional $\mathcal{Q}$ sendo fortemente positivo irá garantir então a minimalidade de $\gamma$ com relação ao funcional $\mathcal{F}$.

\subsection{Curvas na Grassmanniana Metade e Divisível}

Nesta seção vamos descrever de maneira resumida a teoria de curvas na variedade Grassmanniana que está nos trabalhos de Álvarez-Durán ([ÁPD09]) e Durán-Peixoto ([DdAP14]). No trabalho deles um dos objetivos é resolver o problema de congruência de certas curvas, e isto é feito descrevendo os invariantes de tais curvas: duas curvas na Grassmanniana serão congruentes se, e somente se, tiverem os mesmo invariantes. As curvas estudadas em seus trabalhos irão satisfazer uma condição de regularidade genérica, chamada de espalhante (em inglês fanning). Descreveremos um pouco o trabalho deles no que segue.

Primeiramente vamos recordar que se $V$ é um espaço vetorial real então o espaço tangente da Grassmanniana $\operatorname{Gr}(k, V)$ no ponto $\ell$ pode ser canonicamente identificado com o quociente de 
espaços vetoriais $T_{\ell} \operatorname{Gr}(k, V) \cong \operatorname{Hom}(\ell, V / \ell)$.

Definição 1. Seja $I$ um intervalo e $\ell: I \rightarrow \operatorname{Gr}(k, V)$ uma curva na Grassmanniana. O posto de $\ell$ no instante $t \in I$ é o posto de $\ell^{\prime}(t)$ considerado como um elemento de $\operatorname{Hom}(\ell, V / \ell)$.

Vamos fixar uma base de $V$ para então identificar $V \cong \mathbb{R}^{n}$, e assim denotaremos a Grassmanniana por $\operatorname{Gr}(k, n)$. Dada uma curva $\ell(t) \in \operatorname{Gr}(k, n)$ podemos levantar tal curva para um referencial de $k$ vetores l.i. $a_{1}(t), \ldots, a_{k}(t)$ de $\mathbb{R}^{n}$ de modo que eles gerem $\ell(t)$ para cada $t$. Construímos então a seguinte curva de matrizes $\mathcal{A}(t)$ de ordem $n \times k$ onde as colunas de $\mathcal{A}(t)$ são os vetores $a_{i}(t), \mathcal{A}(t)=\left(a_{1}(t)|\ldots| a_{k}(t)\right)$. Sendo $\tilde{\mathcal{A}}(t)$ um outro referencial de $\ell$, a relação entre $\mathcal{A}$ e $\tilde{\mathcal{A}}$ é $\tilde{\mathcal{A}}(t)=\mathcal{A}(t) X(t)$, onde $X(t)$ é uma curva de matrizes $n \times n$ invertível. Temos a seguinte proposição

Proposição 1. Com as notações do parágrafo anterior, teremos

$$
\operatorname{posto}(\ell(t))=\operatorname{posto}\left(\mathcal{A}(t) \mid \mathcal{A}^{\prime}(t)\right)-k
$$

Demonstração. Fixando uma base de $V$ e lembrando de como é a identificação canônica $T_{\ell} G r(k, V) \cong$ $\operatorname{Hom}(\ell, V / \ell)$ : dada a curva $\ell(t)$, escolha matrizes idempotentes $\rho(t)$ que representam projeções tais que a imagem de $\rho(t)$ é $\ell(t)$. Então teremos

- A derivada de $\rho(t)$ é uma curva de endomorfismos que leva $\operatorname{Im}(\rho(t))$ em $\operatorname{ker}(\rho(t))$ e vice-versa.

- O quociente $V / \ell\left(t_{0}\right)$ pode ser identificado com $\operatorname{ker} \rho\left(t_{0}\right)$.

Então a derivada $\rho^{\prime}\left(t_{0}\right)$ fornece um mapa que vai de $\operatorname{Im}\left(\rho\left(t_{0}\right)\right)=\ell\left(t_{0}\right)$ em $\operatorname{ker} \rho\left(t_{0}\right) \cong V / \ell\left(t_{0}\right)$. Se verifica que este mapa é independente da curva de projeções que representa $\ell(t)$.

Fixe uma curva de matrizes $\rho(t)$ como acima. Então, como $\rho(t) \mathcal{A}(t)=\mathcal{A}(t)$ teremos

$$
\mathcal{A}^{\prime}(t)=\rho^{\prime}(t) \mathcal{A}(t)+\rho(t) \mathcal{A}^{\prime}(t) .
$$

Justapondo e calculando o posto chegamos em

$$
\operatorname{posto}\left(\mathcal{A}(t) \mid \mathcal{A}^{\prime}(t)\right)=\operatorname{posto}\left(\mathcal{A}(t) \mid \rho^{\prime}(t) \mathcal{A}(t)+\rho(t) \mathcal{A}^{\prime}(t)\right)=\operatorname{posto}\left(\mathcal{A}(t) \mid \rho^{\prime}(t) \mathcal{A}(t)\right),
$$

onde a última igualdade vem de $\operatorname{Im}(\rho(t))=\ell(t)$ e então as colunas de $\rho(t) \mathcal{A}^{\prime}(t)$ estão no subespaço gerado pelas colunas antes da barra vertical. Agora, como as colunas de $\mathcal{A}(t)$ são a imagem de $\rho(t)$ e as colunas de $\rho^{\prime}(t) \mathcal{A}(t)$ estão no núcleo de $\rho(t)$, segue que o posto da matriz do lado direito da equação acima é $k+\operatorname{posto}\left(\rho^{\prime}(t) \mathcal{A}(t)\right)=k+\operatorname{posto} \ell(t)$.

No caso da Grassmanniana metade, $\operatorname{Gr}(n, 2 n)$, uma curva $\ell: I \rightarrow \operatorname{Gr}(n, 2 n)$ é dita espalhante se tiver posto máximo, isto é se o posto de $\ell(t)$ for igual à $n$ para todo $t$. Isto é equivalente à $\ell^{\prime}(t): \ell(t) \rightarrow \mathbb{R}^{2 n} / \ell(t)$ ser invertível para todo $t$. Esta condição genérica é o ponto de partida para o estudo dos invariantes das curvas espalhantes em [ÁPD09]. Com esta condição é possível definir o endomorfismo fundamental $\mathbf{F}$ dado pela composição $\mathbf{F}(t): \mathbb{R}^{2 n} \rightarrow \mathbb{R}^{2 n} / \ell(t) \stackrel{\left(\ell^{\prime}(t)\right)^{-1}}{\longrightarrow} \ell(t) \hookrightarrow \mathbb{R}^{2 n}$. Lá os autores mostram que os invariantes lineares são completamente descritos por $\mathbf{F}$ e suas derivadas.

Mais precisamente, se $\mathcal{A}(t)$ é um referencial da curva $\ell(t)$ o endomorfismo fundamental do referencial $\mathcal{A}(t)$ no ponto $t_{0}$ pode ser definido pelas equações abaixo devido $\ell$ ser espalhante:

$$
\mathbf{F}\left(t_{0}\right) \mathcal{A}\left(t_{0}\right)=0 \quad \text { e } \quad \mathbf{F}\left(t_{0}\right) \dot{\mathcal{A}}\left(t_{0}\right)=\mathcal{A}\left(t_{0}\right) .
$$

Além disso o endomorfismo fundamental vai satisfazer

- Se $X(t)$ é uma curva de matrizes $n \times n$ invertível, o endomorfismo fundamental do referencial $\mathcal{A}(t) X(t)$ vai coincidir com o endomorfismo fundamental de $\mathcal{A}(t)$.

- Se $\mathbf{T} \in G L(2 n)$ então o endomorfismo fundamental de $\mathbf{T} \mathcal{A}(t)$ será dado pela composição TFT $^{-1}$. 
A primeira propriedade diz que o endomorfismo fundamental é um conceito intríseco à curva $\ell$, isto é, apenas depende de $\ell$, como definido no parágrafo acima.

A derivada do endomorfismo fundamental será uma curva de reflexões e com isso será possível definir a curva de subespaços horizontais de $\ell$

Proposição 2. Seja $\mathbf{F}$ o endomorfismo fundamental do referencial $\mathcal{A}$. A derivada $\dot{\mathbf{F}}(t)$ será uma curva de reflexões de forma que o auto-espaço associado ao auto-valor -1 será gerado pelas colunas de $\mathcal{A}(t)$.

Definição 2. A associação que leva o parâmetro $t$ no núcleo da projeção $\mathbf{P}(t)=(\mathbf{I}-\dot{\mathbf{F}}(t)) / 2$ será chamada de curva horizontal de $\ell$ e denotada por $h$.

Dado um referencial $\mathcal{A}$ podemos construir um referencial da curva horizontal $h$

Definição 3. A derivada horizontal de um referencial espalhante $\mathcal{A}$ é

$$
\mathcal{H}(t)=(\mathbf{I}-\mathbf{P}(t)) \dot{\mathcal{A}}(t) .
$$

O endomorfismo de Jacobi definido abaixo irá medir quanto a curva horizontal se desloca com relação à $\ell$, e será o principal invariante da curva espalhante $\ell$.

Definição 4. Se $\ell$ é espalhante endomorfismo de Jacobi de $\ell$ é definido por $\mathbf{K}(t)=\ddot{\mathbf{F}}(t)^{2} / 4$

Agora sendo $\mathcal{A}(t)$ um referencial espalhante de $\ell$ teremos a seguinte relação diferenciável

$$
\ddot{\mathcal{A}}+\dot{\mathcal{A}} P(t)+\mathcal{A} Q(t)=0,
$$

onde $Q(t)$ e $P(t)$ são curvas de matrizes $n \times n$. Definimos a Schwarziana de $\mathcal{A}(t)$ como sendo

$$
S_{\mathcal{A}}(t)=Q(t)-(1 / 4) P(t)^{2}-\dot{P}(t) / 2 .
$$

(A definição acima é diferente a menos de um fator $1 / 2$ da encontrada em [ÁPD09]).

Teorema 1 (Álvarez-Durán(2009)). A Schwarziana do referencial $\mathcal{A}$ é caracterizada por $\mathbf{K}(t) \mathcal{A}(t)=$ $\mathcal{A}(t) S_{\mathcal{A}}(t)$, e vai satisfazer as seguintes propriedades

- $S e \mathbf{T} \in G L(2 n)$ então $S_{\mathbf{T} \mathcal{A}}(t)=S_{\mathcal{A}}(t)$

- Se $X(t)$ é uma curva de matrizes $n \times n$ invertíveis então $S_{\mathcal{A X}}(t)=(X(t))^{-1} S_{\mathcal{A}}(t) X(t)$,

Com isso podemos associar cada curva espalhante à uma curva de transformações lineares $S_{\ell}$ : $I \rightarrow \operatorname{Lin}(\ell(t) ; \ell(t))$.

O teorema de congruência de curvas espalhantes será dado em termos de um referencial normal de $\ell$.

Definição 5. Um referencial espalhante $\mathcal{B}$ é dito normal se cada coluna de $\ddot{\mathcal{B}}(t)$ for combinação linear das colunas de $\mathcal{B}(t)$ para todo $t$.

Proposição 3. Se $\ell$ é espalhante então existe um referencial normal $\mathcal{B}$ de $\ell$ que pode ser construído a partir de qualquer referencial $\mathcal{A}$, isto é, existe $X(t)$ matriz $n \times n$ invertivel tal que $\mathcal{B}(t)=\mathcal{A}(t) X(t)$. Ainda mais, se $\mathcal{B}$ e $\tilde{\mathcal{B}}$ são dois referenciais normais de $\ell$ então existe uma matriz $n \times n$ invertivel fixa $X_{0}$ tal que $\tilde{\mathcal{B}}(t)=\mathcal{B}(t) X_{0}$.

Teorema 2 (Álvarez-Durán(2009)). Duas curvas espalhantes na Grassmanniana metade Gr(n,2n) serão congruentes se, e somente se, as Schwarzianas de dois referencias normais delas forem conjugadas por uma matriz $n \times n$ fixa invertivel. 
Em [ÁPD09] também é estudado o problema de congruência na Lagrangeana Grassmanniana. Além dos invariantes obtidos do estudo da congruência na Grassmanniana existe um invariante extra, que é discreto, e todos estes invariantes resolvem o problema da congruência.

Em contraste com o problema variacional de primeira ordem (cujo Lagrangeano $L$ depende até o 1-jato das curvas), para ordem $k \geq 2$ (Lagrangeano $L$ depende até o $k$-jato das curvas) iremos construir uma curva de subespaços Lagrangeanos cuja ausência de interseç̧ão com um subespaço fixo irá garantir a positividade de $\mathcal{Q}$. Entretanto tal curva nunca será espalhante no sentido de [ÁPD09], e assim uma outra abordagem deve ser aplicada.

Uma abordagem alternativa seria usando a teoria desenvolvida por Durán-Peixoto ([dAP10], [DdAP14]) na Grassmanniana divisível $\operatorname{Gr}(n, n k)$. No trabalho deles é desenvolvida a teoria dos invariantes de curvas $\ell(t) \in \operatorname{Gr}(n, n k)$, e essa teoria se adapta muito bem na geometria projetiva de equações diferenciais lineares de ordem superior (por exemplo, para a equação de Euler-Lagrange de Lagrangeanos quadráticos!). O conceito de curva espalhante no trabalho deles é dado pelo $(k-1)$ jato da curva $\ell$ : a curva $\ell(t) \in \operatorname{Gr}(n, n k)$ é dita espalhante se dado um referencial $\mathcal{A}$ de $\ell(t)$, isto é, $\mathcal{A}$ é uma matriz $k n \times n$ tal que o espaço gerado pelas colunas é $\ell(t)$ para todo $t$, tivermos que a matriz $\left(\mathcal{A}(t)|\dot{\mathcal{A}}(t)| \cdots \mid \mathcal{A}^{(n-1)}(t)\right)$ é invertível para todo $t$. Note novamente que esta é uma condição genérica de regularidade. Um importante resultado, que é consequência desta teoria de invariantes, é que a bandeira linear definida pela curva $\ell$

$$
\operatorname{span}\{\mathcal{A}(t)\} \subset \operatorname{span}\{\mathcal{A}(t), \dot{\mathcal{A}}(t)\} \subset \ldots
$$

pode ser reduzida à uma bandeira de decomposição, isto é, cada subespaço acima admite um complementar canônico com relação ao anterior. 


\section{Capítulo 2}

\section{Geometria Simplética Projetiva para Funcionais Quadráticos: Caso Unidimensional}

Este capítulo tem o objetivo de introduzir as curvas de Jacobi para problemas de ordem superior no caso de variedades de dimensão 1. Neste caso os cálculos são bem mais simples, dependendo essencialmente de identidades para funcionais quadráticos desenvolvidas por Eswaran [Eas76], ilustrando a ideia geral da passagem para a projetivização e as auto-interseções de planos.

Considere o funcional quadrático de ordem $k$

$$
\mathcal{Q}(h)=\int_{a}^{b} \sum_{i, j=0}^{k} h^{(i)^{T}} L_{i j}(t) h^{(j)} d t=\int_{a}^{b} \sum_{i, j=0}^{k} L_{i j}(t) h^{(i)} h^{(j)} d t
$$

onde $h:[a, b] \rightarrow \mathbb{R}$ e as derivadas até ordem $k-1$ se anulam em $a$ e $b$, isto é, $h^{(i)}(a)=h^{(i)}(b)=0$, $i=0,1, \ldots, k-1$.

Neste caso o funcional quadrático $\mathcal{Q}$ sempre poderá ser escrito em um certo formato, que chamamos de formato padrão, e será da forma

$$
\mathcal{Q}(h)=\int_{a}^{b} \sum_{i=0}^{k} P_{i}(t)\left(h^{(i)}\right)^{2} d t
$$

$\operatorname{com} P_{k}(t)=L_{k k}(t)$.

Para dar uma ideia da mecânica da conta acima do formato padrão vamos deduzi-la no caso $k=1$ e $k=2$.

Caso $k=1$

Teremos o seguinte

$$
\mathcal{Q}(h)=\int_{a}^{b} L_{00} h^{2}+L_{01} h \dot{h}+L_{10} h \dot{h}+L_{11} \dot{h}^{2} d t=\int_{a}^{b} L_{00} h^{2}+\left(L_{01}+L_{10}\right) h \dot{h}+L_{11} \dot{h}^{2} d t .
$$

Integrando por partes o termos do meio e usando a condição nos extremos, $h(a)=h(b)=0$, teremos

$$
\int_{a}^{b}\left(L_{01}+L_{10}\right) h \dot{h} d t=-\frac{1}{2} \int_{a}^{b} \frac{d}{d t}\left(L_{01}+L_{10}\right) h^{2} d t
$$

Logo denotando

$$
P_{0}(t)=L_{00}-\frac{1}{2} \frac{d}{d t}\left(L_{01}+L_{10}\right) \quad \text { e } \quad P_{1}(t)=L_{11}
$$


teremos

$$
\mathcal{Q}(h)=\int_{a}^{b} P_{0}(t) h^{2}+P_{1}(t) \dot{h}^{2} d t
$$

$\underline{\text { Caso } k=2}$

$$
\begin{aligned}
\mathcal{Q}(h)= & \int_{a}^{b} L_{00} h^{2}+L_{10} h \dot{h}+L_{02} h \ddot{h}+L_{01} h \dot{h}+L_{11} \dot{h}^{2}+L_{12} \dot{h} \ddot{h}+L_{02} h \ddot{h}+L_{12} \dot{h} \ddot{h}+L_{22} \ddot{h}^{2} d t= \\
& =\int_{a}^{b}\left(L_{00} h^{2}+\left(L_{10}+L_{01}\right) h \dot{h}+\left(L_{02}+L_{20}\right) h \ddot{h}+L_{11} \dot{h}^{2}+\left(L_{12}+L_{21}\right) \dot{h} \ddot{h}+L_{22} \ddot{h}^{2}\right) d t .
\end{aligned}
$$

Para prosseguir com a conta precisamos fazer algumas integrações por partes, sempre usando a condição nos extremos das funções $h: h(a)=h(b)=\dot{h}(a)=\dot{h}(b)=0$.

Teremos o seguinte

$$
\begin{gathered}
\int_{a}^{b}\left(L_{01}+L_{10}\right) h \dot{h} d t=-\frac{1}{2} \int_{a}^{b} \frac{d}{d t}\left(L_{01}+L_{10}\right) h^{2} d t \\
\int_{a}^{b}\left(L_{12}+L_{21}\right) \dot{h} \ddot{h} d t=-\frac{1}{2} \int_{a}^{b} \frac{d}{d t}\left(L_{12}+L_{21}\right) \dot{h}^{2} d t, \mathrm{e} \\
\int_{a}^{b}\left(L_{02}+L_{20}\right) h \ddot{h} d t=\frac{1}{2} \int_{a}^{b} \frac{d^{2}}{d t^{2}}\left(L_{02}+L_{20}\right) h^{2} d t-\int_{a}^{b}\left(L_{02}+L_{20}\right) \dot{h}^{2} d t .
\end{gathered}
$$

Logo denotando

$$
\begin{gathered}
P_{0}(t)=L_{00}-\frac{1}{2} \frac{d}{d t}\left(L_{01}+L_{10}\right)+\frac{1}{2} \frac{d^{2}}{d t^{2}}\left(L_{02}+L_{20}\right), \\
P_{1}(t)=L_{11}-\frac{1}{2} \frac{d}{d t}\left(L_{12}+L_{21}\right)-\left(L_{02}+L_{20}\right) \mathrm{e} \\
P_{2}(t)=L_{22}
\end{gathered}
$$

teremos

$$
\mathcal{Q}(h)=\int_{a}^{b} P_{0}(t) h^{2}+P_{1}(t) \dot{h}^{2}+P_{2}(t) \ddot{h}^{2} d t .
$$

Os demais casos a conta é a mesma, basta fazer integrações por partes até ficarem os fatores $h$ com derivadas de mesma ordem em cada parcela, e assim obtemos a fórmula (2.1)

Observação 1. Nas contas acima conseguimos chegar sempre no formato padrão porque temos a comutatividade de cada parcela dentro da integral: no caso de $k$ qualquer e $n=1$, estamos trabalhando apenas com funções reais e não matrizes e vetores.

Nos casos de dimensão maior do que 1, a conta para deixar no formato padrão nem sempre é possível. Isto é, ao estudar o problema variacional com Lagrangeano $L: T^{k} Q \rightarrow \mathbb{R} \operatorname{com} \operatorname{dim} Q=$ $n \geq 2$, não podemos agrupar como fizemos acima.

Em [GF00] a conta é feita no caso $k=1$ para $n \geq 2$ qualquer, porém é assumido a simetria do bloco da Hessiana de $L$ que contém as coordenadas das derivadas de ordem 0 e 1 , isto é, os autores assumem o seguinte:

$$
\left(\frac{\partial^{2} L}{\partial q_{0}^{A} \partial q_{1}^{B}}\right)=L_{01}=L_{01}^{T}=\left(\frac{\partial^{2} L}{\partial q_{0}^{B} \partial q_{1}^{A}}\right) .
$$

Para problema variacionais quaisquer não há motivo para assumirmos tais simetrias. Na verdade apenas iremos ter (assumindo suavidade no Lagrangeano $L$ ) que a matriz Hessiana de $L$ será simétrica e não os blocos que possuem derivadas mistas com relação as variáveis $q_{0}^{A}$ e $q_{1}^{B}$, com relação as variáveis $q_{0}^{A}$ e $q_{2}^{B}$, e assim por diante.

Como Gelfand-Fomin mencionam ${ }^{1}$ é possível chegar à condição de minimalidade, que desenvol-

\footnotetext{
${ }^{1}$ Veja a nota de rodapé em [GF00] no capítulo 5, seção 29.1, página 199
} 
veremos a seguir, mesmo sem supor a simetria da matriz acima. Porém a conta fica não trivial.

\subsection{Identidade de Easwaran}

O artigo de Easwaran ([Eas76]) discute condições necessárias e suficientes para o funcional quadrático (2.1) ser positivo definido. Para analisarmos tais condições precisamos do conceito de pontos conjugados.

A equação de Euler-Lagrange do funcional (2.1) acima é dada por

$$
P_{0} h-\frac{d}{d t}\left(P_{1} \dot{h}\right)+\frac{d^{2}}{d t^{2}}\left(P_{2} \ddot{h}\right)+\ldots+(-1)^{k-1} \frac{d^{k-1}}{d t^{k-1}}\left(P_{k-1} h^{(k-1)}\right)+(-1)^{k} \frac{d^{k}}{d t^{k}}\left(P_{k} h^{(k)}\right)=0 .
$$

Quando $\mathcal{Q}$ é a segunda variação de um problema variacional a equação acima é chamada de equação de Jacobi. Note que quando o Lagrangeano $L$ é regular, isto é $P_{k}=L_{k k}=\frac{\partial^{2} L}{\partial q_{k} \partial q_{k}} \neq 0$, a equação acima é uma equação diferenciável de ordem $2 k$.

Seja um conjunto $\left(\sigma_{i}\right)$ de soluções l.i., $i=1, \ldots, k$, de $(2.2)$ que satisfaça

$$
\sigma_{i}^{(j)}(a)=0, \text { para } i=1,2, \ldots, k, \text { e } j=0,1,2, \ldots, k-1,
$$

e defina o seguinte sub-Wronskiano

$$
W\left[\sigma_{1}, \sigma_{2}, \ldots, \sigma_{k}\right](t)=\operatorname{det}\left(\begin{array}{cccc}
\sigma_{1} & \sigma_{2} & \cdots & \sigma_{k} \\
\dot{\sigma_{1}} & \dot{\sigma_{2}} & \cdots & \dot{\sigma_{k}} \\
\vdots & \vdots & \cdots & \vdots \\
\sigma_{1}{ }^{(k-1)} & \sigma_{2}{ }^{(k-1)} & \cdots & \sigma_{k}{ }^{(k-1)}
\end{array}\right)
$$

Definição 6. Um ponto $t^{*} \in(a ; b]$ é dito um ponto conjugado à a se $W\left[\sigma_{1}, \sigma_{2}, \ldots, \sigma_{k}\right]\left(t^{*}\right)=0$.

Observação 2. Note que o conceito de ponto conjugado é um conceito projetivo, isto é, se tivermos outro conjunto $\left(\eta_{i}\right)$ de soluções l.i. de (2.2) satisfazendo as mesma condições (2.3) então

$$
\eta_{i}=\sum_{j=1}^{k} \alpha_{i j} \sigma_{j} \quad i=1, \ldots, k
$$

$\operatorname{com} \alpha_{i j}$ números reais fixos satisfazendo $\operatorname{det}\left(\alpha_{i j}\right) \neq 0$. Portanto

$$
W\left[\eta_{1}, \eta_{2}, \ldots, \eta_{k}\right](t)=W\left[\sigma_{1}, \sigma_{2}, \ldots, \sigma_{k}\right](t) \operatorname{det}\left(\alpha_{i j}\right) .
$$

Para enunciar o seguinte teorema devido à Easwaran vamos supor a condição estrita de Legendre que é: $P_{k}(t)=L_{k k}(t)>0$ para todo $t \in[a, b]$

Teorema 3 (Easwaran(1976)). Suponha que $P_{k}(t)=L_{k k}(t)>0$ para todo $t \in[a, b]$. Se não existem pontos conjugados à a em $(a, b]$ então o funcional $\mathcal{Q}$ é positivo definido, isto é, $\mathcal{Q}(h) \geq 0$ e $\mathcal{Q}(h)=0$ se, e somente se, $h \equiv 0$.

A ideia principal por trás do resultado de Easwaran é identidade enunciada no teorema abaixo. Não iremos demonstrar este teorema pois em um capítulo posterior enunciaremos e demonstraremos uma identidade generalizada que vale em dimensão qualquer.

Teorema 4 (Eastham(1973), Easwaran(1976)). Seja $\sigma_{1}, \ldots, \sigma_{k}$ um conjunto l.i. de soluções de (2.2) satisfazendo (2.3) tal que o sub-Wronskiano satisfaça $W\left[\sigma_{1}, \sigma_{2}, \ldots, \sigma_{k}\right] \neq 0$ no intervalo $(a ; b)$. Então para qualquer $h$ que seja de classe $C^{k}$ temos a identidade

$$
\sum_{i=0}^{k} P_{i}(t)\left(h^{(i)}\right)^{2}=P_{k}\left(\frac{W\left[h, \sigma_{1}, \sigma_{2}, \ldots, \sigma_{k}\right]}{W\left[\sigma_{1}, \sigma_{2}, \ldots, \sigma_{k}\right]}\right)^{2}+\frac{d R}{d t},
$$


onde

$$
W\left[h, \sigma_{1}, \sigma_{2}, \ldots, \sigma_{k}\right]=\operatorname{det}\left(\begin{array}{ccccc}
h & \sigma_{1} & \sigma_{2} & \cdots & \sigma_{k} \\
\dot{h} & \dot{\sigma_{1}} & \dot{\sigma_{2}} & \cdots & \dot{\sigma_{k}} \\
\vdots & \vdots & \vdots & \cdots & \vdots \\
h^{(k-1)} & \sigma_{1}^{(k-1)} & \sigma_{2}^{(k-1)} & \cdots & \sigma_{k}^{(k-1)} \\
h^{(k)} & \sigma_{1}^{(k)} & \sigma_{2}(k) & \cdots & \sigma_{k}{ }^{(k)}
\end{array}\right)
$$

$R$ é uma expressão racional envolvendo $\sigma_{i}, h^{(i)}$ e $P_{i}$, tal que $R\left(t^{*}\right)=0$ se $h^{(i)}\left(t^{*}\right)=0$ para $i=$ $0,1,2, \ldots k-1$.

Assim, nas hipóteses do teorema 4 podemos reescrever o funcional (2.1) como

$$
\mathcal{Q}(h)=\int_{a}^{b} \sum_{i=0}^{k} P_{i}(t)\left(h^{(i)}\right)^{2} d t=\int_{a}^{b}\left(P_{k}\left(\frac{W\left[h, \sigma_{1}, \sigma_{2}, \ldots, \sigma_{k}\right]}{W\left[\sigma_{1}, \sigma_{2}, \ldots, \sigma_{k}\right]}\right)^{2}+\frac{d R}{d t}\right) d t
$$

e o teorema segue. Notemos que, se $\left.\mathcal{Q}(h)=0, W\left[h, \sigma_{1}, \sigma_{2}, \ldots, \sigma_{k}\right]\right)=0$, e então $h$ satisfaz uma equação diferencial de ordem $k$ com todas as condições iniciais até ordem $k-1$ nulas; assim $h \equiv 0$ no intervalo. Observemos também que, tanto neste caso como no caso de dimensão maior, podemos seguir o método usado em [GF00], e considerar para $\epsilon>0$ o funcional perturbado

$$
\mathcal{Q}_{\epsilon}(h)=\int_{a}^{b}\left(P_{k}(t)-\epsilon\right)\left(h^{(k)}\right)^{2}+\sum_{i=0}^{k-1} P_{i}(t)\left(h^{(i)}\right)^{2} d t .
$$

Se $\epsilon$ é suficientemente pequeno, por continuidade o funcional $\mathcal{Q}_{\epsilon}$ satisfaz as hipóteses do teorema 3 em $[a, b]$. Assim a positividade forte segue imediatamente: existe uma constante positiva $\epsilon$ tal que $\mathcal{Q}(h) \geq \epsilon \int_{a}^{b}\left(h^{(k)}\right)^{2}(t) d t$. Devido as condições iniciais $h^{(i)}(a)=0$, para $0 \leq i \leq k-1$, a $k$-ésima derivada domina as outras e existirá constante $C>0$ tal que $\mathcal{Q}(h) \geq C\|h\|_{2, k}^{2}$, onde a norma é a norma $L^{2}$ de Sobolev até ordem $k$ no intervalo $[a, b]$.

Identidades do tipo (2.4) foram estudadas inicialmente por Picone ([Pic10]) no caso de ordem 1 (equação de Euler-Lagrange de ordem 2), seguido por Cimmino, Leighton e Kreith ([Cim30], [Lei70] [Kre73]) no caso de ordem 2 (equação de Euler-Lagrange de ordem 4) e depois, no caso de ordem qualquer por Cimmino, Coppel([Cim39], [Cop71]) e por Eastham ([Eas73]), que apresenta uma demonstração muito elegante neste caso geral. Todos estes resultados são válidos apenas para o caso unidimensional e aparecem ligados à teoria de comparação de Sturm. É importante mencionar Reid ([Rei80]) que desenvolve um identidade geral no caso de sistemas Hamiltonianos que generaliza todas as anteriores. Porém em nenhum destes trabalhos citados aparece o desenvolvimento que trata o funcional quadrático $\mathcal{Q}$ em dimensão qualquer. No capítulo 4 enunciaremos e demonstraremos uma identidade de Picone usando a ideia de Coppel ([Cop71]) no caso unidimensional.

Para finalizarmos esta seção apresentamos o resultado de Eastham da identidade de Picone que é usada na teoria de comparação de Sturm

Teorema 5. Sejam os dois operadores diferenciais de funções reais abaixo

$$
\begin{aligned}
L v & =\sum_{r=0}^{k}(-1)^{r}\left(\frac{d}{d t}\right)^{r}\left(P_{r} v^{(r)}\right) \\
l u & =\sum_{r=0}^{k}(-1)^{r}\left(\frac{d}{d t}\right)^{r}\left(p_{r} u^{(r)}\right)
\end{aligned}
$$

onde $P_{r}$ e $p_{r}$ são funções reais definidas em um intervalo $[a, b]$.

Seja u solução de $l u=0$ e $v_{1}, \ldots, v_{n}$ soluções l.i. de $L v=0$ satisfazendo que as $k-1$ primeiras derivadas se anulam em $t=a$. Se não existir pontos conjugados com respeito à a em $(a, b] d a$ 
equação com respeito à $L$, a seguinte identidade é verdadeira

$$
\frac{d}{d t}(Q(u)-c(u))=\sum_{r=0}^{k}(-1)^{r}\left(p_{r}-P_{r}\right)\left(u^{(r)}\right)^{2}+\left(L_{1} u\right)^{2},
$$

onde $Q(u)$ é uma expressão que depende de $P_{r}$, e de u e suas derivadas, c(u) é uma expressão de depende de $p_{r}$, e de u e suas derivadas, e $L_{1} u$ é dado por

$$
L_{1} u=\frac{P_{n}^{1 / 2}}{W} W\left[v_{1}, \ldots, v_{n}, u\right]
$$

onde $W$ é o sub-Wronskiano com relação à $v_{1}, \ldots, v_{n}$ até a derivada de ordem $k-1$ e $W\left[v_{1}, \ldots, v_{n}, u\right]$ é o sub-Wronskiano com relação à $v_{1}, \ldots, v_{n}, u$ até a derivada de ordem $k$.

\subsection{Curvas de Jacobi}

O teorema 3 tem característica projetiva no sentido da Observação 2. Isso significa que a condição de conjugação depende apenas do subespaço de soluções que se anulam nas primeiras $k$ derivadas.

Isso nos motiva a considerar o seguinte referencial móvel $\mathcal{C}(t)$ de retas em $\mathbb{R}^{2 k}$ : sejam $\sigma_{i}(t)$, $i=1, \ldots, 2 k$, soluções l.i. da equação de Euler-Lagrange (2.2), onde as $k$ primeiras soluções possuem derivadas até ordem $k-1$ iguais à zero no ponto $t=a$, e então construa $\mathcal{C}$ como

$$
\mathcal{C}(t)=\left(\begin{array}{c}
\sigma_{1}(t) \\
\vdots \\
\sigma_{k}(t) \\
\sigma_{k+1}(t) \\
\vdots \\
\sigma_{2 k}(t)
\end{array}\right)
$$

Seja agora $p(t)$ a classe de $\mathcal{C}(t)$ com relação à projeção em $\mathbb{R} \mathrm{P}^{2 k-1}=\operatorname{Gr}(1,2 \mathrm{k})$, isto é, considere a reta gerada por $\mathcal{C}(t)$ em $\mathbb{R}^{2 k}$. Teremos o seguinte teorema

Teorema 6. A curva $p(t)$ é espalhante.

Demonstração. Temos que verificar que a matriz formada por colunas de derivadas até ordem $2 k-1$ do referencial $\mathcal{C}(t)$ é invertível. Assuma o oposto, isto é, existe uma combinação linear não-trivial tal que $\sum_{s=0}^{2 k-1} a_{s} \mathcal{C}^{(s)}(t)=0$. Isso implica que para cada $i=1, \ldots, 2 k$, a mesma relação é satisfeita para cada $\sigma_{i}$, isto é, $\sum_{s=0}^{2 k-1} a_{s} \sigma_{i}^{(s)}(t)=0$. Então teremos um conjunto formado pelas $2 k$ soluções l.i. $\sigma_{i}(t)$ de uma equação diferencial linear com coeficientes constantes de ordem menor que $2 k$, e isso gera uma contradição.

Consideramos agora o referencial formado pelo $k-1$ prolongamento de jatos no referencial $\mathcal{C}$

$$
\mathcal{A}(t)=\left(\begin{array}{cccc}
\sigma_{1}(t) & \sigma_{1}^{\prime}(t) & \ldots & \sigma_{1}^{(k-1)}(t) \\
\vdots & \vdots & \ldots & \vdots \\
\sigma_{k}(t) & \sigma_{1}^{\prime}(t) & \ldots & \sigma_{k}^{(k-1)}(t) \\
\sigma_{k+1}(t) & \sigma_{k+1}^{\prime}(t) & \cdots & \sigma_{k+1}^{(k-1)}(t) \\
\vdots & \vdots & \ldots & \vdots \\
\sigma_{2 k}(t) & \sigma_{2 k}^{\prime}(t) & \ldots & \sigma_{2 k}^{(k-1)}(t)
\end{array}\right)
$$

Definição 7. A curva de Jacobi $\ell(t)$ é a curva gerada pelas colunas de $\mathcal{A}(t)$. 
Segue pelo teorema 6 que $\ell(t)$ tem dimensão $k$, isto é, $\ell$ é uma curva na Grassmanniana $\operatorname{Gr}(k, 2 k)$. Se definirmos o subespaço vertical $\mathcal{V} \subset \mathbb{R}^{2 k}$ como sendo o subespaço dos vetores em $\mathbb{R}^{2 k}$ que possuem as $k$ primeiras coordenadas iguais à zero, teremos que $\mathcal{V}=\ell(a)$, e o teorema 3 se traduz em

Teorema 7. Suponha que $P_{k}(t)=L_{k k}(t)>0$ para todo $t \in[a, b]$. Se $\ell\left(t^{*}\right) \cap \mathcal{V}=\{0\}$ para todo $t^{*} \in(a, b]$, então o funcional $\mathcal{Q}$ é positivo definido.

Em contraste com o caso $k=1$, de problema variacionais de primeira ordem, teremos que $\ell(t)$ não é espalhante, e de fato

Proposição 4. O posto da curva $\ell(t)$ é um.

Demonstração. Segue de imediato o resultado calculando o posto da matriz $(\mathcal{A}(t) \mid \dot{\mathcal{A}}(t))$. Esta matriz possui as colunas de números $k, k+1, \ldots, 2 k-1$ iguais as colunas de números $2, \ldots, k$, respectivamente, e então as $k$ primeiras colunas junto com a última coluna corresponde ao número máximo de colunas li.i. pelo teorema 6 .

Uma outra importante característica da curva $p(t)$ é sua característica simplética. Chegaremos a este resultado após aplicarmos a transformação de Legendre na equação (2.2). Isso será feito, em dimensão qualquer no capítulo 4, e veremos que a bandeira canônica dada pelas prolongações de $p(t)$ será de subespaços isotrópicos, Lagrangeanos e coisotrópicos, dependendo da dimensão. 


\section{Capítulo 3}

\section{Sistemas Hamiltonianos Lineares}

Para o caso de problemas variacionais de dimensões maiores, usaremos a teoria de sistemas Hamiltonianos e a relação com um certo funcional quadrático desenvolvida por Coppel ([Cop71]). Assim vamos enunciar este desenvolvimento e depois chegar ao resultado sobre minimalidade no caso de funcionais quadráticos dados pela Hessiana de problemas variacionais de ordem e dimensão qualquer.

\subsection{Soluções Isotrópicas}

Considere $\left(\mathbb{R}^{2 n}, \omega_{\text {can }}\right)$ com $\omega_{\text {can }}$ a forma simplética canônica, isto é, $\omega_{\text {can }}(x, y)=x^{T} J y$, onde $J$ é a matriz

$$
J=\left(\begin{array}{cc}
0 & \mathrm{Id}_{n} \\
-\mathrm{Id}_{n} & 0
\end{array}\right)
$$

No restante do texto vamos sempre admitir a decomposição de pontos $(y, z)$ de $\mathbb{R}^{2 n}$ com $y$ e $z$ vetores de tamanho $n$. Denotaremos também por $I \in \mathbb{R}$ um intervalo na reta.

Um sistema Hamiltoniano linear em $\mathbb{R}^{2 n}$ é um sistema do seguinte tipo

$$
\left(\begin{array}{c}
\dot{y} \\
\dot{z}
\end{array}\right)=\left(\begin{array}{cc}
A(t) & B(t) \\
C(t) & -A(t)^{T}
\end{array}\right)\left(\begin{array}{l}
y \\
z
\end{array}\right)
$$

onde $A, B, C: I \rightarrow \mathbb{R}^{n \times n}$ são curvas de matrizes quadradas de ordem $n \operatorname{com} B(t)^{T}=B(t)$ e $C(t)^{T}=C(t)$ para todo $t$ e $(y(t), z(t)): I \rightarrow \mathbb{R}^{2 n}$ uma curva em $\mathbb{R}^{2 n}$. As condições dos blocos $B$ e $C$ implicam que a matriz acima estará na álgebra de Lie das matrizes simpléticas.

Definimos também o sistema matricial estendido do sistema acima:

$$
\left(\begin{array}{c}
\dot{Y} \\
\dot{Z}
\end{array}\right)=\left(\begin{array}{cc}
A(t) & B(t) \\
C(t) & -A(t)^{T}
\end{array}\right)\left(\begin{array}{c}
Y \\
Z
\end{array}\right)
$$

com $Y, Z: I \rightarrow \mathbb{R}^{n \times n}$ curvas de matrizes quadradas de ordem $n$. Note que cada coluna da matriz $\left(\begin{array}{l}Y \\ Z\end{array}\right)$ será uma solução do sistema (3.1) acima.

Lema 1. Para soluções $\left(Y_{1}, Z_{1}\right)$ e $\left(Y_{2}, Z_{2}\right)$ de (3.2) teremos que

$$
Y_{1}(t)^{T} Z_{2}(t)-Z_{1}(t)^{T} Y_{2}(t) \equiv C
$$

para todo $t$ onde $C$ é uma matriz constante.

Para demonstrar o lema acima basta derivar $Y_{1}(t)^{T} Z_{2}(t)-Z_{1}(t)^{T} Y_{2}(t)$ e usar que $\left(Y_{1}, Z_{1}\right)$ e $\left(Y_{2}, Z_{2}\right)$ são soluções de (3.2). Em particular se $(Y, Z)$ é solução de (3.2) teremos que

$$
Y(t)^{T} Z(t)-Z(t)^{T} Y(t)
$$


é uma matriz constante.

Definição 8. Uma solução $(Y, Z)$ de (3.2) é dita isotrópica se $Y(t)^{T} Z(t)-Z(t)^{T} Y(t) \equiv 0$.

Observe que $(Y, Z)$ é isotrópica se o espaço gerado pelas colunas de $(Y, Z)$ para cada $t$ for um subespaço isotrópico de $\left(\mathbb{R}^{2 n}, \omega_{\text {can }}\right)$.

Abaixo temos um lema que será usado quando tivermos uma $(Y, Z)$ solução isotrópica e o bloco $Y$ for invertível para todo $t$.

Lema 2. Se $(Y ; Z)$ é uma solução isotrópica de (3.2) com $Y$ invertível para todo $t$ então $Z Y^{-1}$ é simétrica para todo $t$.

Demonstração. Como $(Y ; Z)$ é isotrópica teremos $Y(t)^{T} Z(t)-Z(t)^{T} Y(t)=0$ para todo $t$. Isto implica que $Z(t)(Y(t))^{-1}-\left(Y(t)^{-1}\right)^{T} Z(t)^{T}=0$ para todo o $t$, e por sua vez implica que $\left(Z(t)(Y(t))^{-1}\right)^{T}=$ $Z(t)(Y(t))^{-1}$ para todo $t$.

Uma propriedade de sistemas Hamiltonianos frequente em teoria de controle, e que vai implicar na não existência de pontos conjugados, é o seguinte:

Definição 9. O sistema (3.1) é dito desconjugado no intervalo $I$ se não existir solução não-trivial $(y, z)$ de (3.1) satisfazendo $y\left(t_{1}\right)=y\left(t_{2}\right)=0$ onde $t_{1} \neq t_{2}$ e $t_{1}, t_{2} \in I$.

Mais adiante, quando definirmos pontos conjugados neste contexto, veremos que se um sistema é desconjugado no intervalo $I$ então isso irá implicar que o sistema não possui pontos conjugados em $I$. A recíproca também irá valer se tivermos mais hipóteses.

Antes de continuarmos vamos relembrar o conceito de positividade para matrizes simétricas:

Definição 10. Seja $S$ uma matriz $n \times n$ simétrica:

- Escrevemos $S \geq 0$ se $\eta^{T} S \eta \geq 0$ para todo $\eta \in \mathbb{R}^{n}$, e nesse caso chamamos $S$ de não-negativa.

- Escrevemos $S>0$ se $\eta^{T} S \eta>0$ para todo $\eta \in \mathbb{R}^{n}, \eta \neq 0$, e nesse caso chamamos $S$ de positiva.

- Se $S_{1}$ e $S_{2}$ são matrizes $n \times n$ simétricas escrevemos $S_{1} \geq S_{2}$ se $S_{1}-S_{2} \geq 0$, e $S_{1}>S_{2}$ se $S_{1}-S_{2}>0$. A relação $\geq$ define uma relação de ordem no espaço das matrizes simétricas.

Coppel usa as seguintes condições com relação ao sistema Hamiltoniano (3.1) dadas a seguir e, para simplificar, usaremos a seguinte simbologia:

$(+) B(t) \geq 0$ para todo $t \in I$.

(D) O sistema (3.1) é desconjugado em $I$.

(R) A equação (3.2) tem uma solução isotrópica $(Y, Z)$ tal que $Y(t)$ é invertível para todo $t \in I$.

(C) A única solução $(y ; z)$ de (3.1) tal que y se anula em um subintervalo $J \subset I$ é a solução nula.

Observação 3. A condição (C) é uma condição diferente da usada em [Cop71]. De fato a condição enunciada lá está incorreta. Porém, todos os teoremas seguintes encontrados lá continuando válidos porque a condição $(\mathbf{C})$ assumida aqui neste texto é a que é usada para demonstrar os resultados em [Cop71].

É importante observar também que a condição (C) é chamada de condição de controle e ela aparece na teoria de oscilação de sistemas Hamiltonianos ([Kra95], [dAP10]).

É possível demonstrar as seguintes proposições importantes que relacionam as condições acima. Uma demonstração dos resultados abaixo pode ser encontrada em Coppel ([Cop71])

Proposição 5 ([Cop71]). Supondo o intervalo I compacto e que vale a condição (+). Então (D) $\Rightarrow(R)$.

Proposição 6 ([Cop71]). Supondo que as condições $(+)$ e $(\boldsymbol{C})$ valem, então $(\boldsymbol{R}) \Rightarrow(\boldsymbol{D})$. 


\subsection{Pontos Conjugados}

Vamos supor nesta seção que $I$ é um intervalo aberto e que $(+)$ e $(\mathbf{C})$ valem.

Começamos esta seção com a seguinte observação importante: para todo $t_{0} \in I$ existe um subintervalo $\left[t_{0}-\delta ; t_{0}+\delta\right] \subset I, \delta>0$ tal que o sistema (3.1) é desconjugado em $\left[t_{0}-\delta ; t_{0}+\delta\right]$. De fato, seja uma solução $(Y, Z)$ de $(3.2)$ tal que $Y\left(t_{0}\right)=$ Id e $Z\left(t_{0}\right)=0$ então $Y(t)$ é invertível em alguma vizinhança de $t_{0}$ e a solução $(Y, Z)$ será isotrópica nesta vizinhança. Aplicando a Proposição 6 teremos o resultado.

O conceito de ponto conjugado que veremos mais adiante para problemas variacionais de ordem superior no próximo capítulo vem do conceito generalizado de pontos conjugados de sistemas Hamiltonianos:

Definição 11. Dois pontos $t_{0}, t_{1} \in I, t_{0}<t_{1}$, são ditos conjugados se existir uma solução não-trivial $(y, z)$ do sistema (3.1) tal que $y\left(t_{0}\right)=y\left(t_{1}\right)=0$.

Uma condição equivalente de pontos conjugados pode ser obtida através da equação (3.2). Para isso considere $\left(Y\left(t, t_{0}\right), Z\left(t, t_{0}\right)\right)$ solução isotrópica de (3.2) que satisfaz a condição inicial $\left(Y\left(t_{0}, t_{0}\right), Z\left(t_{0}, t_{0}\right)\right)=(0, \mathrm{Id})$. Assim podemos definir a função de duas variáveis $(Y(t, s), Z(t, s))$ em $I \times I$ com esta propriedade, e ela será contínua em $I \times I$.

Lema 3. Dois pontos $t_{0}, t_{1} \in I$ são conjugados se, e somente se, $\operatorname{det} Y\left(t_{1}, t_{0}\right)=0$.

Uma outra maneira de definir pontos conjugados, seria considerar um referencial $\mathcal{A}(t)=\left(\begin{array}{c}Y(t) \\ Z(t)\end{array}\right)$, onde o conjunto das colunas de $\mathcal{A}$ é um conjunto de soluções l.i. de (3.1) com condição inicial $\mathcal{A}\left(t_{0}\right)=\left(\begin{array}{c}0 \\ \mathrm{Id}\end{array}\right)$.

Considerando os subespaços gerados pelas colunas de $\mathcal{A}(t)$ para cada tempo $t$, este referencial define uma curva $\ell: I \rightarrow \operatorname{Gr}(n ; 2 n) \operatorname{com} \ell\left(t_{0}\right)=\{0\} \times \mathbb{R}^{n}$. Note que na verdade a curva $\ell$ é uma curva na Lagrangeana Grassmanniana, $\ell: I \rightarrow \Lambda_{n}$. Com isso teremos então a seguinte caracterização de pontos conjugados:

Lema 4. O ponto $t_{1} \in I$ é conjugados à $t_{0}$ se, e somente se, $\ell\left(t_{0}\right) \cap \ell\left(t_{1}\right) \neq\{0\}$.

Para desenvolver a equivalência de pontos conjugados e desconjugação precisamos de mais alguns resultados destes conceitos.

Seja $t_{0} \in I$. Denotaremos por $\sigma\left(t_{0}\right)=\sup \left\{c \in I, c>t_{0}\right.$; o sistema (3.1) é desconjugado em $\left.\left[t_{0} ; c\right]\right\}$. Note que se $\sigma\left(t_{0}\right)$ estiver bem definido, isto é, se $\sigma\left(t_{0}\right)<+\infty$, então para todo $t_{0}^{\prime}<t_{0} \operatorname{com} t_{0}^{\prime} \in I$, $\sigma\left(t_{0}^{\prime}\right)$ também estará bem definido. E teremos ainda que $\sigma\left(t_{0}^{\prime}\right) \leq \sigma\left(t_{0}\right)$.

Seja ainda $t_{0} \in I$. Suponha que exista um ponto $t^{*} \in I, t^{*}>t_{0}$ tal que $\operatorname{det} Y\left(t^{*}, t_{0}\right)=0$. Definiremos $\omega\left(t_{0}\right)$ como sendo o menor destes valores $t^{*}$, caso exista.

A relação entre $\sigma\left(t_{0}\right)$ e $\omega\left(t_{0}\right)$ é dada a seguir, juntamente com algumas propriedades de $\sigma$ :

Proposição 7. As seguintes afirmações são verdadeiras:

1. Seja $t_{0} \in I$. Caso $\sigma\left(t_{0}\right)$ e $\omega\left(t_{0}\right)$ existam teremos que $\sigma\left(t_{0}\right)=\omega\left(t_{0}\right)$.

2. A função $\sigma$ é crescente em seu dominio.

3. A função $\sigma$ é contínua e seu dominio é um subintervalo aberto de I.

Observação 4. Suponha $[a, b] \subset I$ e também a existência de pelo menos um ponto conjugado á $a$, e que $\omega(a)$ exista. É óbvio que se o sistema (3.1) for desconjugado em $[a ; b]$ então não existirá nenhum ponto conjugado à $a$ no intervalo $(a ; b]$. Reciprocamente, se não existir nenhum ponto conjugado á $a$ no intervalo $(a ; b]$ teremos que $\omega(a)>b$ e pela Proposição 7 obtemos $\sigma(a)>b$. Com isso existe $c$ tal que $b<c \leq \sigma(a)$ tal que (3.1) é desconjugado em [a;c] e isso implicará que (3.1) é desconjugado em $[a ; b]$. 
Portanto nas condições do parágrafo acima teremos

O sistema (3.1) é desconjugado em $[a ; b]$

$\Uparrow$

Não existem pontos conjugados à $a$ em $(a ; b]$.

\subsection{Funcional Quadrático Hamiltoniano}

A seguir apresentaremos os resultados em [Cop71] à respeito do sinal de um certo funcional quadrático que é definido por um sistema Hamiltoniano.

Seja $I=[a ; b]$ e o funcional quadrático

$$
\tilde{\mathcal{Q}}(y ; z)=\int_{a}^{b} z^{T} B z+y^{T} C y d t .
$$

Definição 12. Diremos que um par $(y, z)$ é admissivel se $(y, z)$ satisfizer $\dot{y}=A(t) y+B(t) z$ com $y(a)=y(b)=0$.

Definição 13. $\tilde{\mathcal{Q}}$ é dito não-negativo se $\tilde{\mathcal{Q}} \geq 0$ para todos $(y ; z)$ admissiveis. $\tilde{\mathcal{Q}}$ é dito positivo se $\tilde{\mathcal{Q}}$ for não-negativo, e se $\tilde{\mathcal{Q}}=0$ para $\operatorname{algum}(y ; z)$ admissível implicar que $y \equiv 0$ no intervalo $I$.

Teremos os seguinte teoremas que relacionam as condições de desconjugação de (3.1) com o sinal de $\tilde{\mathcal{Q}}$

Teorema 8 ([Cop71]). Se $(+)$ e $(\boldsymbol{D})$ valem em I então $\tilde{\mathcal{Q}}$ é positivo para todos os pares $(y ; z)$ admissiveis.

A prova deste teorema será dada no próximo capítulo como aplicação da identidade de Picone.

Teorema 9 ([Cop71]). Se $(C)$ vale e $\tilde{\mathcal{Q}}$ é positivo para todos os pares admissiveis então $(+)$ e (D) valem em $I$. 


\section{Capítulo 4}

\section{Minimalidade e Curva de Isotrópicos}

Ao estudar problemas variacionais, a resposta para a pergunta de quando uma curva será ponto crítico de um dado funcional é dada pelas equações de Euler-Lagrange. Para verificar a minimalidade de tal ponto crítico temos que analisar a Hessiana do funcional dado no ponto crítico. Em particular, o ponto crítico será um ponto de mínimo se a Hessiana for fortemente positiva definida.

Com as considerações feitas no capítulo 1, sem perda de generalidade, vamos desenvolver aqui a análise da Hessiana do seguinte funcional

$$
c \mapsto \mathcal{F}(c)=\int_{a}^{b} L\left(j^{(k)} c(t)\right) d t
$$

com $L: T^{k} \mathbb{R}^{n} \rightarrow \mathbb{R}$ sendo o Lagrangeano. A Hessiana de $\mathcal{F}$ em um ponto crítico $\gamma:[a ; b] \rightarrow \mathbb{R}^{n}$ de $\mathcal{F}$ é um funcional quadrático definido nos campos $h:[a, b] \rightarrow \mathbb{R}^{n}$ ao longo de $\gamma$ dado por

$$
\delta^{2} \mathcal{F}(h)=\int_{a}^{b} \sum_{i, j=0}^{k} h^{(i)^{T}} L_{i j}(t) h^{(j)} d t
$$

onde $h:[a ; b] \rightarrow \mathbb{R}^{n}$ satisfazem $j^{s} h(a)=j^{s} h(b)=0$ para $0 \leq s \leq k-1$ (chamadas de curvas admissíveis), e as matrizes $L_{i j}(t)$ correspondem à blocos da Hessiana de $L$ calculada ao longo de $\gamma$, isto é,

$$
L_{i j}(t)=\left(\frac{\partial^{2} L}{\partial q_{i}^{A} \partial q_{j}^{B}}\left(j^{k} \gamma(t)\right)\right)_{A B} .
$$

As condições $j^{s} h(a)=j^{s} h(b)=0$, para $0 \leq s \leq k-1$, traduzem o fato que estamos estudando problemas variacionais com extremos fixos até o $(k-1)$-jato, portanto as variações $h$ de $\gamma$ todas terão o $(k-1)$-jato igual a zero nos pontos $a$ e $b$.

Nosso objetivo ao longo da seção será estudar as condições para o funcional quadrático

$$
\mathcal{Q}(h)=\int_{a}^{b} \sum_{i, j=0}^{k} h^{(i)^{T}} L_{i j}(t) h^{(j)} d t
$$

ser positivo definido, com curvas $h:[a, b] \rightarrow \mathbb{R}^{n}$ satisfazendo $j^{s} h(a)=j^{s} h(b)=0$, para $0 \leq s \leq k-1$, onde $L_{i j}:[a, b] \rightarrow \mathbb{R}^{n \times n}$ são curvas de matrizes para $i, j=0, \ldots k$.

Neste capítulo apresentaremos uma curva de subespaços isotrópicos que irá medir pontos conjugados, e portanto a minimalidade dos problemas variacionais.

No restante do capítulo vamos assumir, quando for necessário, a regularidade do funcional quadrático, isto é, det $L_{k k} \neq 0$, e a condição estrita de Legendre, isto é, $L_{k k}$ positiva definida. 


\subsection{Identidade de Picone}

Primeiramente, vamos enunciar e demonstrar a identidade de Picone para dimensão qualquer no contexto Hamiltoniano. Esta identidade será uma generalização da identidade (2.4), que será vista na próxima seção.

Teorema 10 (Identidade de Picone Generalizada). Com notações do capitulo anterior, seja $(Y ; Z)$ solução isotrópica de (3.2) com $Y(t)$ invertivel para todo t. Considere também $(y ; z)$ satisfazendo $\dot{y}=A(t) y+B(t) z$. Nestas condições teremos que

$$
\frac{d}{d t}\left(y^{T} Z Y^{-1} y\right)=z^{T} B z+y^{T} C y-\left(z-Z Y^{-1} y\right)^{T} B\left(z-Z Y^{-1} y\right)
$$

Demonstração. Efetuando diretamente a conta do lado esquerdo da identidade e usando as hipóteses teremos

$$
\begin{aligned}
& \frac{d}{d t}\left(y^{T} Z Y^{-1} y\right)=\dot{y}^{T} Z Y^{-1} y+y^{T} \dot{Z} Y^{-1} y+y^{T} Z\left(-Y^{-1} \dot{Y} Y^{-1}\right) y+y^{T} Z Y^{-1} \dot{y}= \\
& =(A y+B z)^{T} Z Y^{-1} y+y^{T}\left(C Y-A^{T} Z\right) Y^{-1} y-y^{T} Z\left(Y^{-1}(A Y+B Z) Y^{-1}\right) y+y^{T} Z Y^{-1}(A y+B z)= \\
& =\left(y^{T} A+z^{T} B\right) Z Y^{-1} y+y^{T}\left(C-A^{T} Z Y-1\right) y-y^{T} Z\left(Y^{-1} A+Y^{-1} B Z Y^{-1}\right) y+y^{T} Z Y^{-1}(A y+B z)= \\
& =z^{T} B Z Y^{-1} y+y^{T} C y-y^{T} Z Y^{-1} B Z Y^{-1} y+y^{T} Z Y^{-1} B z+z^{T} B z-z^{T} B z= \\
& =z^{T} B+y^{T} C y+\underbrace{\left(z^{T} B Z Y^{-1} y-y^{T} Z Y^{-1} B Z Y^{-1} y+y^{T} Z Y^{-1} B z-z^{T} B z\right)}_{\Delta} .
\end{aligned}
$$

Agora desenvolvendo o último termo da identidade (4.3) acima e usando que $Z Y^{-1}$ é simétrica (Lema 2) teremos

$$
\begin{aligned}
-\left(z-Z Y^{-1} y\right)^{T} B\left(z-Z Y^{-1} y\right) & =\left(y^{T}\left(Z Y^{-1}\right)^{T}-z^{T}\right) B\left(z-Z Y^{-1} y\right)= \\
& =\left(y^{T} Z Y^{-1}-z^{T}\right) B\left(z-Z Y^{-1} y\right)= \\
& =y^{T} Z Y^{-1} B z-y^{T} Z Y^{-1} B Z Y^{-1} y-z^{T} B z+z^{T} B Z Y^{-1} y=\Delta,
\end{aligned}
$$

portanto a identidade segue.

Podemos aplicar a identidade (4.3) para provar o Teorema 8:

Demonstração do Teorema 8. Supondo que valem $(+)$ e (D), pela proposição (5) podemos encontrar uma solução $(Y ; Z)$ isotrópica de $(3.2)$ tal que $Y(t)$ é invertível para todo $t$. Pelo teorema acima a identidade (4.3) vale para todos pares $(y ; z)$ admissíveis.

Podemos então escrever o funcional quadrático (3.3) acima como

$$
\begin{aligned}
\tilde{\mathcal{Q}} & =\int_{a}^{b} z^{T} B z+y^{T} C y d t=\int_{a}^{b} \frac{d}{d t}\left(y^{T} Z Y^{-1} y\right)+\left(z-Z Y^{-1} y\right)^{T} B\left(z-Z Y^{-1} y\right) d t= \\
& =\int_{a}^{b}\left(z-Z Y^{-1} y\right)^{T} B\left(z-Z Y^{-1} y\right) d t \geq 0,
\end{aligned}
$$

para todos os pares admissíveis $(y ; z)$.

Se $\tilde{\mathcal{Q}}=\int_{a}^{b}\left(z-Z Y^{-1} y\right)^{T} B\left(z-Z Y^{-1} y\right) d t=0$ para um certo par admissível $(y ; z)$ teremos que $B\left(z-Z Y^{-1} y\right)=0$. Como $\dot{y}=A y+B z$ isso implica na equação anterior que $\dot{y}-\left(A+B Z Y^{-1}\right) y=0$. Como $y$ satisfaz $y(a)=0$ teremos que ter $y \equiv 0$ no intervalo $I$, e então $\tilde{\mathcal{Q}}$ é positivo.

\subsection{Identidade de Picone na Reta}

A identidade de Picone (4.3) generaliza as identidades (2.4) encontradas no artigo de Easwaran quando trabalhamos no caso $n=1$. Neste caso, o funcional quadrático (4.2) é o funcional visto em 
(2.1), que no formato padrão, é dado por

$$
\mathcal{Q}(h)=\int_{a}^{b} \sum_{i=0}^{k} P_{i}(t)\left(h^{(i)}\right)^{2} d t,
$$

onde $h:[a, b] \rightarrow \mathbb{R}$ e $h^{(i)}(a)=h^{(i)}(b)=0, i=0,1, \ldots, k-1$, e $P_{k}(t)=L_{k k}(t)$. Iremos verificar tal generalização nos casos $k=1$ e $k=2$ mais adiante.

Primeiramente usaremos a transformação de Legendre para escrever a equação (2.2) como um sistema Hamiltoniano. Para uma definição formal da transformação de Legendre, consulte o apêndice A.2.6

Para um Lagrangeano regular $L$ a transformação de Legendre Leg $: \mathbb{R}^{2 k} \rightarrow \mathbb{R}^{2 k}$ calculada no $(2 k-1)$-jato de uma função $h$ é dada por

$$
\operatorname{Leg}\left(h, \dot{h}, \ldots, h^{(k-1)}, h^{(k)}, \ldots, h^{(2 k-1)}\right)=(y, z)
$$

com

$$
y=\left(\begin{array}{c}
h \\
\dot{h} \\
\ddot{h} \\
\vdots \\
h^{(k-1)}
\end{array}\right) \quad \text { e } \quad z=\left(\begin{array}{c}
z_{1} \\
z_{2} \\
z_{3} \\
\vdots \\
z_{k}
\end{array}\right)
$$

onde

$$
z_{i}=\sum_{j=i}^{k}(-1)^{j-i}\left(\frac{d}{d t}\right)^{j-i}\left(P_{j} h^{(j)}\right) .
$$

Neste caso teremos o seguinte sistema Hamiltoniano, que é equivalente à equação (2.2),

$$
\left(\begin{array}{l}
\dot{y} \\
\dot{z}
\end{array}\right)=\left(\begin{array}{cc}
A(t) & B(t) \\
C(t) & -A(t)^{T}
\end{array}\right)\left(\begin{array}{l}
y \\
z
\end{array}\right)
$$

com $A(t), B(t)$ e $C(t)$ dadas por

$$
A(t)=\left(\begin{array}{cccccc}
0 & 1 & 0 & \cdots & 0 & 0 \\
0 & 0 & 1 & \cdots & 0 & 0 \\
\vdots & \vdots & \vdots & \ddots & \vdots & \vdots \\
0 & 0 & 0 & \cdots & 1 & 0 \\
0 & 0 & 0 & \cdots & 0 & 1 \\
0 & 0 & 0 & \cdots & 0 & 0
\end{array}\right), \quad B(t)=\left(\begin{array}{cccccc}
0 & 0 & 0 & \cdots & 0 & 0 \\
0 & 0 & 0 & \cdots & 0 & 0 \\
\vdots & \vdots & \vdots & \vdots & \vdots & \vdots \\
0 & 0 & 0 & \cdots & 0 & 0 \\
0 & 0 & 0 & \cdots & 0 & 0 \\
0 & 0 & 0 & \cdots & 0 & \left(P_{k}\right)^{-1}
\end{array}\right)
$$

e

$$
C(t)=\left(\begin{array}{cccccc}
P_{0} & 0 & 0 & \cdots & 0 & 0 \\
0 & P_{1} & 0 & \cdots & 0 & 0 \\
\vdots & \vdots & \ddots & \vdots & \vdots & \vdots \\
0 & 0 & 0 & \ddots & 0 & 0 \\
0 & 0 & 0 & \cdots & P_{k-2} & 0 \\
0 & 0 & 0 & \cdots & 0 & P_{k-1}
\end{array}\right)
$$

Observe que $B(t) \geq 0$ se $P_{k}>0$.

Seja $\sigma_{1}, \ldots, \sigma_{k}$ um conjunto l.i. de soluções de (2.2) satisfazendo

$$
\sigma_{i}^{(j)}(a)=0, \text { para } i=1,2, \ldots, k, \text { e } j=0,1, \ldots, k-1 .
$$

Considere agora o par de matrizes $(Y, Z)$ dado pela transformação de Legendre das soluções 
$\sigma_{1}, \ldots, \sigma_{k}$, isto é

$$
Y=\left(\begin{array}{cccc}
\sigma_{1} & \sigma_{2} & \cdots & \sigma_{k} \\
\dot{\sigma_{1}} & \dot{\sigma_{2}} & \cdots & \dot{\sigma_{k}} \\
\vdots & \vdots & \cdots & \vdots \\
\sigma_{1}{ }^{(k-1)} & \sigma_{2}{ }^{(k-1)} & \cdots & \sigma_{k}^{(k-1)}
\end{array}\right) \quad \text { e } \quad Z=\left(\begin{array}{cccc}
z_{1}^{1} & z_{1}^{2} & \cdots & z_{1}^{k} \\
z_{2}^{1} & z_{2}^{2} & \cdots & z_{2}^{k} \\
\vdots & \vdots & \cdots & \vdots \\
z_{k}^{1} & z_{k}^{2} & \cdots & z_{k}^{k}
\end{array}\right)
$$

onde $z_{i}^{j}$ é dado por

$$
z_{i}^{j}=\sum_{l=i}^{k}(-1)^{l-i}\left(\frac{d}{d t}\right)^{l-i}\left(P_{l} \sigma_{j}^{(l)}\right) .
$$

Definimos também os seguintes sub-Wronskianos $W\left[\sigma_{1}, \sigma_{2}, \ldots, \sigma_{k}\right]=\operatorname{det} Y \mathrm{e}$

$$
W\left[h, \sigma_{1}, \sigma_{2}, \ldots, \sigma_{k}\right]=\operatorname{det}\left(\begin{array}{ccccc}
h & \sigma_{1} & \sigma_{2} & \cdots & \sigma_{k} \\
\dot{h} & \dot{\sigma_{1}} & \dot{\sigma}_{2} & \cdots & \dot{\sigma}_{k} \\
\vdots & \vdots & \vdots & \cdots & \vdots \\
h^{(k-1)} & \sigma_{1}{ }^{(k-1)} & \sigma_{2}{ }^{(k-1)} & \cdots & \sigma_{k}{ }^{(k-1)} \\
h^{(k)} & \sigma_{1}{ }^{(k)} & \sigma_{2}{ }^{(k)} & \cdots & \sigma_{k}{ }^{(k)}
\end{array}\right)
$$

com $h$ uma função de classe $C^{k}$. Com isso já podemos demonstrar a identidade de Picone na reta (2.4). Assumindo as hipóteses do teorema 4 teremos

Demonstração do teorema 4. Observe que se tivermos $W\left[\sigma_{1}, \sigma_{2}, \ldots, \sigma_{k}\right] \neq 0$ no intervalo $(a ; b)$ então a solução $(Y ; Z)$ será isotrópica com $Y(t)$ invertível para todo $t \in(a ; b)$. Observe também que para o sistema Hamiltoniano acima todo par da forma

$$
(y ; z)=\left(y_{1}, \ldots, y_{k} ; z_{1}, \ldots, z_{k}\right)=\left(h, \ldots, h^{(k-1)} ; 0, \ldots, 0, P_{k} h^{(k)}\right)
$$

com $h$ de classe $C^{k}$ irá satisfazer a equação da primeira linha de blocos do sistema, isto é, $\dot{y}=$ $A(t) y+B(t) z$. Portanto estamos nas hipóteses do teorema da identidade de Picone e vale (4.3)

$$
z^{T} B z+y^{T} C y-\left(z-Z Y^{-1} y\right)^{T} B\left(z-Z Y^{-1} y\right)=\frac{d}{d t}\left(y^{T} Z Y^{-1} y\right) .
$$

Não é difícil mostrar que as primeiras duas parcelas acima irão corresponder a somária da identidade (2.4),

$$
z^{T} B z+y^{T} C y=\sum_{l=0}^{k} P_{l}(t)\left(h^{(l)}\right)^{2} .
$$

O termo do lado direito da igualdade, dentro do sinal de derivada, corresponderá a expressão racional $R$

$$
y^{T} Z Y^{-1} y=R,
$$

e note que $R$ irá satisfazer as propriedade enunciadas no teorema 4. A parte que envolve mais contas de combinatória consiste em mostrar que

$$
\left(z-Z Y^{-1} y\right)^{T} B\left(z-Z Y^{-1} y\right)=P_{k}\left(\frac{W\left[h, \sigma_{1}, \sigma_{2}, \ldots, \sigma_{k}\right]}{W\left[\sigma_{1}, \sigma_{2}, \ldots, \sigma_{k}\right]}\right)^{2} .
$$

Vamos verificar isso para os casos $k=1$ e $k=2$.

Caso $k=1$ : A parte esquerda de (4.5) fica

$$
\left(P_{1} \dot{h}-P_{1} \dot{\sigma}_{1} \sigma_{1}{ }^{-1} h\right) P_{1}^{-1}\left(P_{1} \dot{h}-P_{1} \dot{\sigma}_{1} \sigma_{1}^{-1} h\right)=P_{1}\left(\dot{h}-\dot{\sigma}_{1} \sigma_{1}{ }^{-1} h\right) .
$$


E a parte da direita de (4.5) fica

$$
P_{1}\left(\frac{W\left[h, \sigma_{1}\right]}{W\left[\sigma_{1}\right]}\right)^{2}=P_{1}\left(\frac{h \dot{\sigma_{1}}-\dot{h} \sigma_{1}}{\sigma_{1}}\right)^{2}=P_{1}\left(\dot{h}-\dot{\sigma_{1} \sigma_{1}}{ }^{-1} h\right) .
$$

Portanto neste caso segue.

Caso $k=2:$ O termo $\left(z-Z Y^{-1} y\right)$ fica

$$
\begin{aligned}
\left(z-Z Y^{-1} y\right) & =\left(\begin{array}{l}
z_{1} \\
z_{2}
\end{array}\right)-\left(\begin{array}{ll}
z_{1}^{1} & z_{1}^{2} \\
z_{2}^{1} & z_{2}^{2}
\end{array}\right) \frac{1}{\operatorname{det} Y}\left(\begin{array}{ll}
C_{11} & C_{21} \\
C_{12} & C_{22}
\end{array}\right)\left(\begin{array}{l}
y_{1} \\
y_{2}
\end{array}\right)= \\
& =\left(\begin{array}{l}
z_{1} \\
z_{2}
\end{array}\right)-\frac{1}{\operatorname{det} Y}\left(\begin{array}{l}
z_{1}^{1} C_{11} y_{1}+z_{1}^{1} C_{21} y_{2}+z_{1}^{2} C_{12} y_{1}+z_{1}^{2} C_{22} y_{2} \\
z_{2}^{1} C_{11} y_{1}+z_{2}^{1} C_{21} y_{2}+z_{2}^{2} C_{12} y_{1}+z_{2}^{2} C_{22} y_{2}
\end{array}\right),
\end{aligned}
$$

onde a matriz com elementos $C$ é a matriz transposta dos cofatores de $Y$. Como a matriz $B$ neste caso é

$$
B=\left(\begin{array}{cc}
0 & 0 \\
0 & P_{2}^{-1}
\end{array}\right)
$$

teremos que a parte esquerda de (4.5) fica

$$
\begin{aligned}
& \left(z-Z Y^{-1} y\right)^{T} B\left(z-Z Y^{-1} y\right)=P_{2}^{-1}\left(z_{2}-\frac{1}{\operatorname{det} Y}\left(z_{2}^{1} C_{11} y_{1}+z_{2}^{1} C_{21} y_{2}+z_{2}^{2} C_{12} y_{1}+z_{2}^{2} C_{22} y_{2}\right)\right)^{2}= \\
& =P_{2}^{-1}\left(P_{2} \ddot{h}-\frac{1}{\operatorname{det} Y}\left(P_{2} \ddot{\sigma_{1}} \dot{\sigma}_{2} h+P_{2} \ddot{\sigma}_{1}\left(-\sigma_{2}\right) \dot{h}+P_{2} \ddot{\sigma}_{2}\left(-\dot{\sigma_{1}}\right) h+P_{2} \ddot{\sigma}_{2} \sigma_{1} \dot{h}\right)\right)^{2}= \\
& =\frac{P_{2}}{(\operatorname{det} Y)^{2}}\left(\ddot{h} \operatorname{det} Y-\ddot{\sigma}_{1} \dot{\sigma}_{2} h+\ddot{\sigma}_{1} \sigma_{2} \dot{h}+\ddot{\sigma}_{2} \dot{\sigma_{1}} h-\ddot{\sigma}_{2} \sigma_{1} \dot{h}\right)^{2}= \\
& =\frac{P_{2}}{(\operatorname{det} Y)^{2}}\left(\ddot{h} \operatorname{det} Y-\dot{h}\left(\ddot{\sigma}_{2} \sigma_{1}-\ddot{\sigma}_{1} \sigma_{2}\right)+h\left(\ddot{\sigma}_{2} \dot{\sigma}_{1}-\ddot{\sigma}_{1} \dot{\sigma}_{2}\right)\right)^{2} .
\end{aligned}
$$

A parte da direita de (4.5) fica o mesmo resultado que acima basta desenvolver o determinante $W\left[h, \sigma_{1}, \sigma_{2}\right]$. Portanto resultado neste caso segue.

\subsection{Da Equação de Jacobi para Sistemas Hamiltonianos}

Usando as ideias da seção anterior conseguiremos transformar toda equação de Jacobi em um sistema Hamiltoniano, e então obteremos o resultado para dimensão $n$ qualquer. Conseguiremos assim, encontrar condições que garantam a positividade da Hessiana de tais problemas com dimensão $n$ e ordem $k$ quaisquer.

Antes disso vamos lembrar da fórmula do funcional quadrático que estamos estudando:

$$
\mathcal{Q}(h)=\int_{a}^{b} \sum_{i, j=0}^{k} h^{(i)^{T}} L_{i j}(t) h^{(j)} d t
$$

com $h:[a, b] \rightarrow \mathbb{R}^{n}$ satisfazendo $j^{s} h(a)=j^{s} h(b)=0$, para $0 \leq s \leq k-1$, e vamos supor que $\mathcal{Q}$ é um funcional regular, isto é, $\operatorname{det} L_{k k} \neq 0$.

Note que, diferentemente do caso $n=1$, não podemos escrever o funcional quadrático (4.6) no formato padrão como foi feito em (2.1). Isso por que as matrizes $L_{i j}$ não serão simétricas. Porém podemos deixá-lo em um formato mais simples que facilitará as contas posteriores. Vale observar que o resultado não depende de escrever o funcional no formato abaixo, isto é, não é essencial. Porém as contas ficam mais simples em tal formato. Vamos deduzir tais contas nos casos $k=1 \mathrm{e}$ $k=2$. 


\section{Caso $k=1$ :}

$$
\mathcal{Q}(h)=\int_{a}^{b} h^{T} L_{00} h+h^{T} L_{01} \dot{h}+\dot{h}^{T} L_{10} h+\dot{h}^{T} L_{11} \dot{h} d t=\int_{a}^{b} \frac{1}{2} h^{T} M_{00} h+h^{T} M_{01} \dot{h}+\frac{1}{2} \dot{h}^{T} M_{11} \dot{h} d t,
$$

com

$$
M_{00}=2 L_{00}, \quad M_{01}=L_{01}+L_{10}^{T}, \quad M_{11}=2 L_{11}
$$

\section{Caso $k=2:$}

$$
\mathcal{Q}(h)=\int_{a}^{b} h^{T} L_{00} h+h^{T}\left(L_{01}+L_{10}^{T}\right) \dot{h}+h^{T}\left(L_{02}+L_{20}^{T}\right) \ddot{h}+\dot{h}^{T} L_{11} \dot{h}+\dot{h}^{T}\left(L_{12}+L_{21}^{T}\right) \ddot{h}+\ddot{h}^{T} L_{22} \ddot{h} d t .
$$

Usando que $h$ é admissível e integração por partes, podemos reescrever um dos termos acima como

$$
\int_{a}^{b} h^{T}\left(L_{02}+L_{20}^{T}\right) \ddot{h} d t=-\int_{a}^{b} \dot{h}^{T}\left(L_{02}+L_{20}^{T}\right) \dot{h} d t-\int_{a}^{b} h^{T} \frac{d}{d t}\left(L_{02}+L_{20}^{T}\right) \dot{h} d t .
$$

Logo o funcional quadrático acima fica

$$
\mathcal{Q}(h)=\int_{a}^{b} \frac{1}{2} h^{T} M_{00} h+h^{T} M_{01} \dot{h}+\frac{1}{2} \dot{h}^{T} M_{11} \dot{h}+2 \dot{h}^{T} M_{12} \ddot{h}+\frac{1}{2} \ddot{h}^{T} M_{22} \ddot{h} d t,
$$

com

$$
\begin{gathered}
M_{00}=2 L_{00}, \quad M_{01}=L_{01}+L_{10}^{T}-\frac{d}{d t}\left(L_{02}+L_{20}^{T}\right), \quad M_{11}=2\left(L_{11}-L_{02}-L_{20}^{T}\right), \quad M_{12}=L_{12}+L_{21}^{T}, \\
M_{22}=2 L_{22} .
\end{gathered}
$$

De modo geral, para $k$ qualquer, podemos escrever o funcional quadrático, através de manipulações análogas, como

$$
\mathcal{Q}(h)=\int_{a}^{b} \tilde{L}\left(t, h, \dot{h}, \ddot{h}, \ldots, h^{(k)}\right) d t
$$

com

$$
\tilde{L}=\frac{1}{2} \sum_{i=0}^{k} h^{(i)^{T}} M_{i i} h^{(i)}+\sum_{i=0}^{k-1} h^{(i)^{T}} M_{i(i+1)} h^{(i+1)} \quad \text { e } \quad M_{k k}=L_{k k} .
$$

Note ainda que, sem perda de generalidade, podemos supor as matrizes $M_{i i}$ simétricas já que a parte anti-simétrica de $M_{i i}$ não contribui para a conta do funcional, isto é:

$$
\int_{a}^{b} h^{(i)^{T}} M_{i i} h^{(i)} d t=\int_{a}^{b} h^{(i)^{T}} M_{i i}^{T} h^{(i)} d t \Rightarrow \int_{a}^{b} h^{(i)^{T}}\left(M_{i i}-M_{i i}^{T}\right) h^{(i)} d t=0 .
$$

Logo teremos o seguinte

$$
Q(h)=\int_{a}^{b} \tilde{L}\left(t, h, \dot{h}, \ddot{h}, \ldots, h^{(k)}\right) d t
$$

com

$$
\tilde{L}=\frac{1}{2} \sum_{i=0}^{k} h^{(i)^{T}} M_{i i} h^{(i)}+\sum_{i=0}^{k-1} h^{(i)^{T}} M_{i(i+1)} h^{(i+1)}, \quad M_{i i}=M_{i i}^{T} \quad \text { e } \quad M_{k k}=L_{k k} .
$$

Da mesma forma que na primeira seção, iremos considerar a equação de Euler-Lagrange (chamada de equação de Jacobi se vier de um problema variacional) do funcional quadrático (4.7):

$$
\frac{\partial \tilde{L}}{\partial q_{0}}\left(j^{k} h(t)\right)-\frac{d}{d t}\left(\frac{\partial \tilde{L}}{\partial q_{1}}\left(j^{k} h(t)\right)\right)+\ldots+(-1)^{k} \frac{d^{k}}{d t^{k}}\left(\frac{\partial \tilde{L}}{\partial q_{k}}\left(j^{k} h(t)\right)\right)=0 .
$$


As derivadas parciais na fórmula acima podem ser obtidas diretamente pela definição de $\tilde{L}$ :

$$
\begin{gathered}
\frac{\partial \tilde{L}}{\partial q_{0}}=M_{00} h+M_{01} \dot{h}, \\
\frac{\partial \tilde{L}}{\partial q_{j}}=M_{j j} h^{(j)}+M_{(j-1) j}^{T} h^{(j-1)}+M_{j(j+1)} h^{(j+1)}, \text { para } j=1, \ldots, k-1, \\
\frac{\partial \tilde{L}}{\partial q_{k}}=M_{k k} h^{(k)}+M_{(k-1) k}^{T} h^{(k-1)} .
\end{gathered}
$$

Uma outra maneira de escrever as relações acima e que será útil em contas posteriores é o seguinte

$$
\left(\begin{array}{c}
\frac{\partial L}{\partial q_{0}} \\
\vdots \\
\frac{\partial L}{\partial q_{k-1}}
\end{array}\right)=C(t)\left(\begin{array}{c}
h \\
\vdots \\
h^{(k-1)}
\end{array}\right)+\left(\begin{array}{c}
0 \\
\vdots \\
M_{(k-1) k} M_{k k}^{-1} \frac{\partial L}{\partial q_{k}}
\end{array}\right)
$$

onde $C$ é uma curva de matrizes $k n \times k n$ possuindo os blocos $M_{i j}$ da seguinte forma

$$
C(t)=\left(\begin{array}{cccccc}
M_{00} & M_{01} & 0 & 0 & \ldots & 0 \\
M_{01}^{T} & M_{11} & M_{12} & 0 & \ldots & 0 \\
0 & M_{12}^{T} & M_{22} & M_{23} & \ldots & 0 \\
\vdots & \vdots & \ddots & \ddots & \ddots & \vdots \\
0 & \cdots & 0 & M_{(k-2)(k-1)}^{T} & M_{(k-2)(k-2)} & M_{(k-2)(k-1)} \\
0 & \cdots & 0 & 0 & M_{(k-2)(k-1)}^{T} & M_{(k-1)(k-1)}-M_{(k-1) k} M_{k k}^{-1} M_{(k-1) k}^{T}
\end{array}\right)
$$

\subsection{Transformação de Legendre}

Para transformar a equação (4.8) acima em um sistema Hamiltoniano teremos que usar a transformação de Legendre. A transformação de Legendre será uma função Leg $: \mathbb{R}^{2 k n} \rightarrow \mathbb{R}^{2 k n}$ que pode ser definida a partir do Lagrangeano $\tilde{L}$ com o formalismo encontrado em [dLR85] (veja apêndice A.2.6).

A transformação de Legendre aplicada ao $(2 k-1)$-jato de uma curva $h$ será dada por

$$
\operatorname{Leg}\left(h, \dot{h}, \ldots, h^{(k-1)}, h^{(k)}, \ldots, h^{(2 k-1)}\right)=(y, z)
$$

com

$$
y=\left(\begin{array}{c}
h \\
\dot{h} \\
\ddot{h} \\
\vdots \\
h^{(k-1)}
\end{array}\right) \quad \text { e } \quad z=\left(\begin{array}{c}
z_{1} \\
z_{2} \\
z_{3} \\
\vdots \\
z_{k}
\end{array}\right)
$$

onde

$$
z_{i}=\sum_{j=i}^{k}(-1)^{j-i}\left(\frac{d}{d t}\right)^{j-i}\left(\frac{\partial \tilde{L}}{\partial q_{j}}\right)
$$

com $\tilde{L}=\tilde{L}\left(h, \dot{h}, \ldots, h^{(k)}\right)$

Não é difícil verificar que Leg será invertível, se e somente se, $M_{k k}=L_{k k}$ for invertível. Na 
verdade a transformação de Legendre aplicada ao $(2 k-1)$-jato de $h$ pode ser escrita como

$$
\operatorname{Leg}\left(h, \ldots, h^{(2 k-1)}\right)=\left(\begin{array}{c}
y \\
z
\end{array}\right)=\left(\begin{array}{cc}
\text { Id } & 0 \\
B_{1} & B_{2}
\end{array}\right)\left(\begin{array}{c}
h \\
\vdots \\
h^{(2 k-1)}
\end{array}\right)
$$

onde os blocos Id, $0, B_{1}$ e $B_{2}$ são matrizes $k n \times k n$. O bloco $B_{1}$ será uma matriz triangular superior por blocos, e o bloco $B_{2}$ terá como blocos $M_{k k}$ ou $-M_{k k}$ na diagonal secundária e zeros abaixo desta diagonal, isto é, $B_{2}$ é da forma

$$
B_{2}=\left(\begin{array}{ccccc}
* & * & \cdots & * & (-1)^{k-1} M_{k k} \\
* & * & \cdots & (-1)^{k-2} M_{k k} & 0 \\
\vdots & \vdots & . & \vdots & \vdots \\
* & -M_{k k} & 0 & \cdots & 0 \\
M_{k k} & 0 & 0 & \cdots & 0
\end{array}\right)
$$

Disto tiramos que cada coordenada $z_{i}$ vai depender (linearmente) somente de $h^{(i-1)}, h^{(i)}, \ldots, h^{(2 k-i)}$, para $i=1, \ldots, k$.

A transformação de Legendre levará soluções $h$ da equação (4.8) em soluções $(y, z)$ do sistema Hamiltoniano abaixo

$$
\left(\begin{array}{l}
\dot{y} \\
\dot{z}
\end{array}\right)=\left(\begin{array}{cc}
A(t) & B(t) \\
C(t) & -A(t)^{T}
\end{array}\right)\left(\begin{array}{l}
y \\
z
\end{array}\right)
$$

onde $C(t)$ é a mesma matriz encontrada em (4.9), $A(t)$ é dada por

$$
A(t)=\left(\begin{array}{cccccc}
0 & \mathrm{Id} & 0 & \cdots & 0 & 0 \\
0 & 0 & \mathrm{Id} & \cdots & 0 & 0 \\
\vdots & \vdots & \vdots & \ddots & \vdots & \vdots \\
0 & 0 & 0 & \cdots & \mathrm{Id} & 0 \\
0 & 0 & 0 & \cdots & 0 & \mathrm{Id} \\
0 & 0 & 0 & \cdots & 0 & -M_{k k}^{-1} M_{(k-1) k}^{T}
\end{array}\right)
$$

onde os blocos Id tem tamanho $n \times n$, e $B(t)$ é dada por

$$
B(t)=\left(\begin{array}{cccccc}
0 & 0 & 0 & \cdots & 0 & 0 \\
0 & 0 & 0 & \cdots & 0 & 0 \\
\vdots & \vdots & \vdots & \vdots & \vdots & \vdots \\
0 & 0 & 0 & \cdots & 0 & 0 \\
0 & 0 & 0 & \cdots & 0 & 0 \\
0 & 0 & 0 & \cdots & 0 & \left(M_{k k}\right)^{-1}
\end{array}\right)
$$

Pelas expressões acima teremos que $B(t)^{T}=B(t)$ e $C(t)^{T}=C(t)$, e então a matriz que tem os blocos $A, B$ e $C$ na expressão (4.12) estará na álgebra de Lie simplética.

Uma outra observação encontrada em [Cop71], e que será útil adiante, é que se tivermos uma função $h \in C^{k}\left([a, b], \mathbb{R}^{n}\right)$ e definirmos o par $(y, z)$ por

$$
y=\left(\begin{array}{c}
h \\
\dot{h} \\
\vdots \\
h^{(k-2)} \\
h^{(k-1)}
\end{array}\right) \quad \text { e } \quad z=\left(\begin{array}{c}
z_{1} \\
z_{2} \\
\vdots \\
z_{k-1} \\
z_{k}
\end{array}\right)=\left(\begin{array}{c}
0 \\
0 \\
\vdots \\
0 \\
M_{k k} h^{(k)}+M_{(k-1) k}^{T} h^{(k-1)}
\end{array}\right) \text {, }
$$


então a relação abaixo é uma identidade

$$
\dot{y}=A(t) y(t)+B(t) z(t) .
$$

Isto pode ser provado diretamente através das definições das matrizes $A(t)$ e $B(t)$ acima.

Para terminar esta seção, novamente seguindo as ideias de [Cop71], vamos deduzir duas expressões que serão úteis para escrever o funcional $\mathcal{Q}$ de outra maneira. Seja o par $(y, z)$ definido por (4.13) para uma função $h \in C^{k}\left([a, b], \mathbb{R}^{n}\right)$. Teremos

$$
\begin{aligned}
z^{T} B z & =\left(\begin{array}{lll}
z_{1}^{T} & \cdots & z_{k}^{T}
\end{array}\right)\left(\begin{array}{cccccc}
0 & 0 & 0 & \cdots & 0 & 0 \\
0 & 0 & 0 & \cdots & 0 & 0 \\
\vdots & \vdots & \vdots & \vdots & \vdots & \vdots \\
0 & 0 & 0 & \cdots & 0 & 0 \\
0 & 0 & 0 & \cdots & 0 & 0 \\
0 & 0 & 0 & \cdots & 0 & \left(M_{k k}\right)^{-1}
\end{array}\right)\left(\begin{array}{c}
z_{1} \\
\vdots \\
z_{k}
\end{array}\right)=z_{k}^{T} M_{k k}^{-1} z_{k}= \\
& =\left(\begin{array}{ll}
h^{(k)^{T}} M_{k k}+h^{(k-1)^{T}} M_{(k-1) k}
\end{array}\right) M_{k k}^{-1}\left(M_{k k} h^{(k)}+M_{(k-1) k}^{T} h^{(k-1)}\right)= \\
& =h^{(k)^{T}} M_{k k} h^{(k)}+2 h^{(k-1)^{T}} M_{(k-1) k} h^{(k)}+h^{(k-1)^{T}} M_{(k-1) k} M_{k k}^{-1} M_{(k-1) k}^{T} h^{(k-1)},
\end{aligned}
$$

e

$$
\begin{aligned}
y^{T} C y & =y^{T}\left(\left(\begin{array}{c}
\frac{\partial L}{\partial q_{0}} \\
\vdots \\
\frac{\partial L}{\partial q_{k-1}}
\end{array}\right)-\left(\begin{array}{c}
0 \\
\vdots \\
M_{(k-1) k} M_{k k}^{-1} \frac{\partial L}{\partial q_{k}}
\end{array}\right)\right)=y^{T}\left(\begin{array}{c}
\frac{\partial L}{\partial q_{0}} \\
\vdots \\
\frac{\partial L}{\partial q_{k-1}}
\end{array}\right)-y^{T}\left(\begin{array}{c}
0 \\
\vdots \\
M_{(k-1) k} M_{k k}^{-1} \frac{\partial L}{\partial q_{k}}
\end{array}\right)= \\
& =\sum_{i=0}^{k-1} h^{(i)^{T}} M_{i i} h^{(i)}+2 \sum_{i=0}^{k-2} h^{(i)^{T}} M_{i(i+1)}+h^{(k-1)^{T}} M_{(k-1) k} h^{(k)}-h^{(k-1)^{T}} M_{(k-1) k} M_{k k}^{-1} \frac{\partial L}{\partial q_{k}}= \\
& =\sum_{i=0}^{k-1} h^{(i)^{T}} M_{i i} h^{(i)}+2 \sum_{i=0}^{k-2} h^{(i)^{T}} M_{i(i+1)} h^{(i+1)}+h^{(k-1)^{T}} M_{(k-1) k} h^{(k)} \\
& -\left(h^{(k-1)^{T}} M_{(k-1) k} h^{(k)}+h^{(k-1)^{T}} M_{(k-1) k} M_{k k}^{-1} M_{(k-1) k}^{T} h^{(k-1)}\right)= \\
& =\sum_{i=0}^{k-1} h^{(i)^{T}} M_{i i} h^{(i)}+2 \sum_{i=0}^{k-2} h^{(i)^{T}} M_{i(i+1)} h^{(i+1)}-h^{(k-1)} M_{(k-1) k} M_{k k}^{-1} M_{(k-1) k}^{T} h^{(k-1)} .
\end{aligned}
$$

Somando $z^{T} B z \operatorname{com} y^{T} C y$ nos leva à

$$
z^{T} B z+y^{T} C y=\sum_{i=0}^{k} h^{(i)^{T}} M_{i i} h^{(i)}+2 \sum_{i=0}^{k-1}{h^{(i)^{T}}} M_{i(i+1)} h^{(i+1)}=2 \tilde{L} .
$$

Pelas contas acima, teremos a seguinte igualdade dos funcionais quadráticos para funções $h \in$ $C^{k}\left([a, b], \mathbb{R}^{n}\right)$ :

$$
\tilde{\mathcal{Q}}=\int_{a}^{b} z^{T} B z+y^{T} C y d t=2 \mathcal{Q}=2 \int_{a}^{b} \tilde{L}\left(t, \dot{h}(t), \ddot{h}(t), \ldots h^{(k)}(t)\right) d t .
$$

Podemos resumir esta seção com os teoremas 11 e 12 abaixo. A demonstração do teorema 11 se dá através da transformação de Legendre, substituindo (4.10) na equação (4.12). A demonstração do teorema 12 é feita pelas equações e identidades (4.13), (4.14), (4.15), (4.16) e (4.17)

Teorema 11. Uma função $h \in C^{2 k}\left([a, b], \mathbb{R}^{n}\right)$ é solução de (4.8) se, e somente se, a imagem do $(2 k-1)$-jato de $h$ pela transformação de Legendre é solução de (4.12). 
Teorema 12. Seja $h \in C^{k}\left([a, b], \mathbb{R}^{n}\right)$ e considere o par $(y, z)$ dado por

$$
y=\left(\begin{array}{c}
h \\
\dot{h} \\
\vdots \\
h^{(k-2)} \\
h^{(k-1)}
\end{array}\right) \quad e \quad z=\left(\begin{array}{c}
z_{1} \\
z_{2} \\
\vdots \\
z_{k-1} \\
z_{k}
\end{array}\right)=\left(\begin{array}{c}
0 \\
0 \\
\vdots \\
0 \\
M_{k k} h^{(k)}+M_{(k-1) k}^{T} h^{(k-1)}
\end{array}\right) .
$$

Então a seguinte identidade se verifica

$$
\dot{y}=A(t) y+B(t) z(t),
$$

e temos a seguinte igualdade de funcionais quadráticos

$$
\tilde{\mathcal{Q}}=\int_{a}^{b} z^{T} B z+y^{T} C y d t=2 \mathcal{Q}=2 \int_{a}^{b} \tilde{L}\left(t, \dot{h}(t), \ddot{h}(t), \ldots h^{(k)}(t)\right) d t
$$

onde $A, B$ e $C$ são curvas de matrizes descritas anteriormente.

\subsection{Positividade de $\mathcal{Q}$}

Antes de demonstrarmos o teorema principal do nosso trabalho, que irá dar condições suficientes para $\mathcal{Q}$ ser positivo, vamos definir o conceito de pontos conjugados para problemas de ordem superior.

Seja $h_{1}, \ldots, h_{k n}$ um conjunto l.i. de soluções de (4.8) tal que todas as derivadas até ordem $(k-1)$ se anulam no ponto $t=a$. Considere o seguinte sub-Wronskiano

$$
W\left[h_{1}, \ldots, h_{k n}\right](t)=\operatorname{det}\left(\begin{array}{cccc}
h_{1}^{T}(t) & {\dot{h_{1}}}^{T}(t) & \cdots & h_{1}^{(k-1)^{T}}(t) \\
h_{2}^{T}(t) & {\dot{h_{2}}}^{T}(t) & \cdots & h_{2}^{(k-1)^{T}}(t) \\
\vdots & \vdots & \vdots & \vdots \\
h_{k n}^{T}(t) & \dot{h}_{k n}^{T}(t) & \cdots & h_{k n}^{(k-1)^{T}}(t)
\end{array}\right)
$$

Definição 14. Um ponto $t^{*} \in(a, b]$ é dito ponto conjugado à $a$ da equação (4.8) se

$$
W\left[h_{1}, \ldots, h_{k n}\right]\left(t^{*}\right)=0 .
$$

De forma equivalente, podemos caracterizar pontos conjugados através do lema

Lema 5. Um ponto $t^{*}$ é conjugado à a se, e somente se, existir uma solução não nula $h_{0}$ de (4.8) tal que $h_{0}^{(i)}(a)=h_{0}^{(i)}\left(t^{*}\right)=0$, para $i=0,1, \ldots, k-1$.

Agora, como no caso unidimensional, temos nosso resultado principal, dado pelo seguinte teorema

Teorema 13. Supondo a condição de Legendre estrita $\left(L_{k k}>0\right)$ e que não existem pontos conjugados à a em $(a, b]$, então o funcional $\mathcal{Q}$ é positivo definido para todo $h \in C^{k}\left([a, b], \mathbb{R}^{n}\right)$ com $h^{(i)}(a)=h^{(i)}(b)=0$ para $i=0,1, \ldots, k-1$. Isto é, $\mathcal{Q}(h) \geq 0$, e $\mathcal{Q}(h)=0$ somente quando $h \equiv 0$.

Demonstração. Para provar usaremos a identidade de Picone generalizada (4.3) para o sistema (4.12).

Antes, considere os coeficientes da E.D.O. (4.8), de ordem $2 k$, definidos em um intervalo um pouco maior, digamos, $[a-\epsilon, b]$, de modo que:

- $M_{k k}=L_{k k}$ é positiva definida em $[a-\epsilon, b]$, e 
- não existem pontos conjugados à $a-\epsilon$ em $(a-\epsilon, b]$.

Podemos supor isso pela dependência contínua de E.D.Os. com o parâmetro inicial e porque as duas condições acima são abertas.

Fixe agora um conjunto $\left\{h_{1}, \ldots, h_{k n}\right\}$ de soluções l.i. de (4.8) satisfazendo $h_{j}^{(i)}(a-\epsilon)=0$ para $i=0,1, \ldots, k-1$ e $j=1, \ldots k n$, e então considere a imagem pela transformação de Legendre de cada $(2 k-1)$-jato de $h_{j}$

$$
\operatorname{Leg}\left(h_{j}, \dot{h_{j}}, \ldots, h_{j}^{(2 k-1)}\right)=\left(\begin{array}{c}
\mu_{j} \\
\zeta_{j}
\end{array}\right)
$$

e construa a matriz $2 k n \times k n$ onde as colunas são as imagens acima

$$
\left(\begin{array}{c}
Y(t) \\
Z(t)
\end{array}\right)=\left(\begin{array}{ccc}
\mu_{1} & \cdots & \mu_{k n} \\
\zeta_{1} & \cdots & \zeta_{k n}
\end{array}\right)
$$

O bloco $Y$ de tamanho $k n \times k n$ acima corresponde à projeção nas primeiras $k n$ coordenadas da transformação de Legendre, e o bloco $Z$ de tamanho $k n \times k n$ corresponde à projeção nas últimas $k n$ coordenadas da transformação de Legendre, isto é

$$
Y(t)=\left(\begin{array}{lll}
\mu_{1} & \cdots & \mu_{k n}
\end{array}\right) \text { and } Z(t)=\left(\begin{array}{lll}
\zeta_{1} & \cdots & \zeta_{k n}
\end{array}\right) .
$$

Pelo fato que a condição inicial é satisfeita $Y(a-\epsilon)=0$, teremos pelo lema 1

$$
Y^{T}(t) Z(t)-Z(t)^{T} Y(t)=0, \forall t \in[a-\epsilon, b]
$$

E, como não existem pontos conjugados à $a-\epsilon$ em $[a, b]$, teremos também que $Y(t)$ é invertível para todo $t \in[a, b]$. Logo $(Y, Z)$ é uma solução isotrópica do sistema matricial associado à (4.8) com $Y(t)$ invertível para todo $t \in[a ; b]$.

Pelo teorema 12, estamos nas hipóteses do teorema 10. Portanto usando a identidade (4.3) com

$$
y=\left(\begin{array}{c}
h \\
\dot{h} \\
\vdots \\
h^{(k-2)} \\
h^{(k-1)}
\end{array}\right) \quad \text { e } \quad z=\left(\begin{array}{c}
z_{1} \\
z_{2} \\
\vdots \\
z_{k-1} \\
z_{k}
\end{array}\right)=\left(\begin{array}{c}
0 \\
0 \\
\vdots \\
0 \\
M_{k k} h^{(k)}+M_{(k-1) k}^{T} h^{(k-1)}
\end{array}\right)
$$

teremos

$$
\begin{aligned}
2 \mathcal{Q}=\tilde{\mathcal{Q}} & =\int_{a}^{b} z^{T} B z+y^{T} C y d t=\int_{a}^{b} \frac{d}{d t}\left(y^{T} Z Y^{-1} y\right)+\left(z-Z Y^{-1} y\right)^{T} B\left(z-Z Y^{-1} y\right) d t= \\
& =\int_{a}^{b}\left(z-Z Y^{-1} y\right)^{T} B\left(z-Z Y^{-1} y\right) d t \geq 0 .
\end{aligned}
$$

Usando as condições iniciais

$$
h^{(i)}(a)=h^{(i)}(b)=0 \quad \text { para } i=0,1, \ldots, k-1 \Leftrightarrow y(a)=y(b)=0,
$$

vai implicar que

$$
\begin{gathered}
2 \mathcal{Q}=\tilde{\mathcal{Q}}=0 \Leftrightarrow \int_{a}^{b}\left(z-Z Y^{-1} y\right)^{T} B\left(z-Z Y^{-1} y\right) d t=0 \Leftrightarrow B\left(z-Z Y^{-1} y\right)=0 \Leftrightarrow \\
\dot{y}-A y-B Z Y^{-1} y=0 \Leftrightarrow \dot{y}=\left(A+B Z Y^{-1}\right) y \Leftrightarrow y \equiv 0 .
\end{gathered}
$$

Portanto o teorema segue. 


\subsection{Curvas de Jacobi}

Da mesma forma que no caso unidimensional, o desenvolvimento acima nos motiva a definir o seguinte referencial de tamanho $2 k n \times n$

$$
\mathcal{A}(t)=\left(\begin{array}{c}
h_{1}^{T} \\
\vdots \\
h_{k n}^{T} \\
h_{k n+1}^{T} \\
\vdots \\
h_{2 k n}^{T}
\end{array}\right),
$$

onde $\left\{h_{1}, \ldots, h_{2 k n}\right\}$ é um conjunto fundamental (isto é, são l.i.) de soluções de (4.8) tais que, para $i=1, \ldots, k n$, cada $h_{i}$ tem todas as derivadas até ordem $k-1$ iguais à zero em $t=a$.

Novamente, considerando o espaço $p(t)$ gerado pelas colunas de $\mathcal{A}$ para cada $t$, teremos uma curva de subespaços que vai ser espalhante no sentido generalizado de [DdAP14]. Para verificarmos isso, considere a prolongamento ao $(2 k-1)$-jato de $p$ escrito em termos do referencial $\mathcal{A}$. E então o posto deste prologamento será máximo, isto é, a matriz $2 k n \times 2 k n$ abaixo será não-degenerada

$$
\left(\mathcal{A}(t)|\dot{\mathcal{A}}(t)| \cdots \mid \mathcal{A}^{(2 k-1)}(t)\right)
$$

para cada $t$.

Teorema 14. A curva $p(t):[a, b] \rightarrow G r(n, 2 k n)$ é espalhante.

Considerando agora o prolongamento do $(k-1)$-jato de $p$, teremos uma curva de subespaços $\ell$ gerada pelas colunas da matriz $2 k n \times k n$ abaixo

$$
\left(\mathcal{A}(t)|\dot{\mathcal{A}}(t)| \cdots \mid \mathcal{A}^{(k-1)}(t)\right) .
$$

Definição 15. A curva de Jacobi $\ell:[a, b] \rightarrow \operatorname{Gr}(k n, 2 k n)$ é a curva de subespaços gerada pelas colunas de $\mathcal{A}(t)$.

Agora se definirmos o espaço vertical $\mathcal{V}_{k n}^{2 k n} \subset \mathbb{R}^{2 k}$ como sendo o subespaço de vetores que possuem as $k n$ primeiras coordenadas nulas, teremos que $\mathcal{V}_{k n}^{2 k n}=\ell(a)$ e o teorema 13 se traduz em

Teorema 15. Suponha que $L_{k k}(t)>0$ para todo $t \in[a, b]$. Se $\ell\left(t^{*}\right) \cap \mathcal{V}_{k n}^{2 k n}=\{0\}$ para todo $t^{*} \in(a, b]$, então o funcional $\mathcal{Q}(t)$ é positivo definido.

\subsection{Prolongamentos Simpléticos}

Outra propriedade que a curva espalhante $p:[a, b] \rightarrow \operatorname{Gr}(n, 2 k n)$ irá satisfazer é

- o prolongamento $j^{s} p, s=0, \ldots, k-2$, será uma curva de subespaços isotrópicos com relação à $\left(\mathbb{R}^{2 k n}, \omega_{\text {can }}\right)$

- o prolongamento $\ell=j^{(k-1)} p$, será uma curva de Lagrangeanos com relação à $\left(\mathbb{R}^{2 k n}, \omega_{\text {can }}\right)$

- o prolongamento $j^{s} p, s=k, \ldots, 2 k-1$, será uma curva de subespaços coisotrópicos com relação à $\left(\mathbb{R}^{2 k n}, \omega_{\text {can }}\right)$

Para justificarmos isto considere a transformação de Legendre aplicada ao $(2 k-1)$-jato de $p$. Pela equação (4.11) teremos

$$
\operatorname{Leg}\left(h, \ldots, h^{(2 k-1)}\right)=\left(\begin{array}{c}
y \\
z
\end{array}\right)=\left(\begin{array}{cc}
\text { Id } & 0 \\
B_{1} & B_{2}
\end{array}\right)\left(\begin{array}{c}
h \\
\vdots \\
h^{(2 k-1)}
\end{array}\right)
$$


onde $B_{1}$ é triangular superior e $B_{2}$ é da forma

$$
B_{2}=\left(\begin{array}{ccccc}
* & * & \ldots & * & (-1)^{k-1} M_{k k} \\
* & * & \cdots & (-1)^{k-2} M_{k k} & 0 \\
\vdots & \vdots & . & \vdots & \vdots \\
* & -M_{k k} & 0 & \cdots & 0 \\
M_{k k} & 0 & 0 & \cdots & 0
\end{array}\right)
$$

Agora para cada $h_{i}$ em (4.18) considere a imagem $\left(\mu_{i}, \zeta_{i}\right)$ pela transformação de Legendre do $(2 k-1)$-jato de $h_{i}$

$$
\operatorname{Leg}\left(h_{i}, \ldots, h_{i}^{(2 k-1)}\right)=\left(\begin{array}{c}
\mu_{i} \\
\zeta_{i}
\end{array}\right)
$$

e construa a seguinte matriz

$$
\left(\begin{array}{ccc}
\mu_{1} & \cdots & \mu_{2 k n} \\
\zeta_{1} & \cdots & \zeta_{2 k n}
\end{array}\right)=\left(\begin{array}{cc}
\operatorname{Id} & 0 \\
B_{1} & B_{2}
\end{array}\right)\left(\begin{array}{ccc}
h_{1} & \cdots & h_{2 k n} \\
\vdots & \vdots & \vdots \\
h_{1}^{(2 k-1)} & \cdots & h_{2 k n}^{(2 k-1)}
\end{array}\right) .
$$

Calculando a transposta na relação acima teremos

$$
\begin{aligned}
\left(\begin{array}{cc}
\mu_{1}^{T} & \zeta_{1}^{T} \\
\vdots & \vdots \\
\mu_{2 k n}^{T} & \zeta_{2 k n}^{T}
\end{array}\right) & =\left(\mathcal{A}(t)|\dot{\mathcal{A}}(t)| \cdots \mid \mathcal{A}^{(2 k-1)}(t)\right)\left(\begin{array}{cc}
\text { Id } & B_{1}^{T} \\
0 & B_{2}^{T}
\end{array}\right) \\
& =\left(\mathcal{A}(t)|\dot{\mathcal{A}}(t)| \cdots\left|\mathcal{A}^{(k-1)}(t)\right| \mathcal{C}^{1}(t)|\cdots| \mathcal{C}^{k}(t)\right)
\end{aligned}
$$

onde os blocos $\mathcal{C}^{i}$ de tamanho $2 k n \times n$ terão como colunas combinações lineares das colunas de $\mathcal{A}^{(i-1)}, \ldots, \mathcal{A}^{(2 k-i)}$, para $i=1, \ldots, k$, e podem ser escritos como

$$
\mathcal{C}^{i}=\mathcal{A}^{(i-1)} Q_{i-1}^{i}+\ldots+\mathcal{A}^{(2 k-i)} Q_{2 k-i}^{i},
$$

onde $Q_{j}^{i}$ são matrizes $n \times n$ e, o mais importante, $Q_{2 k-i}^{i}= \pm M_{k k}$.

A matriz do lado esquerdo da igualdade (4.19) estará na grupo de Lie das matrizes simpléticas supondo a condição inicial

$$
\left(\begin{array}{cc}
\mu_{1}^{T}(a) & \zeta_{1}^{T}(a) \\
\vdots & \vdots \\
\mu_{2 k n}^{T}(a) & \zeta_{2 k n}^{T}(a)
\end{array}\right)=\left(\begin{array}{cc}
0_{k n} & \operatorname{Id}_{k n} \\
-\operatorname{Id}_{k n} & 0_{k n}
\end{array}\right)
$$

que pode ser imposta, e vai condizer com as condições iniciais de $h_{i}$, pelo fato da transformação de Legendre ser invertível. Teremos então que as matrizes em (4.19) são simpléticas para cada $t$. Denotando por $J$ a matriz da forma simplética canônica $\omega_{\text {can }}$ em $\mathbb{R}^{2 k n}$, segue que

$$
\left(\mathcal{A}(t)|\cdots| \mathcal{C}^{k}(t)\right)^{T} J\left(\mathcal{A}(t)|\cdots| \mathcal{C}^{k}(t)\right)=J=\left(\begin{array}{cc}
0_{k n} & \operatorname{Id}_{k n} \\
-\operatorname{Id}_{k n} & 0_{k n}
\end{array}\right) .
$$

A relação (4.20) acima implica

$$
\mathcal{A}^{(i-1)^{T}} J \mathcal{A}^{(j-1)}=0
$$

para $i, j=1, \ldots, k, \mathrm{e}$

$$
\mathcal{C}^{i T} J \mathcal{A}^{(j-1)}=0
$$


para $2 \leq i \leq k$ e $1 \leq j \leq i-1$. Desenvolvendo a segunda expressão e usando que $\mathcal{C}^{i}$ é escrito como

$$
\mathcal{C}^{i}=\mathcal{A}^{(i-1)} Q_{i-1}^{i}+\ldots+\mathcal{A}^{(2 k-i)} Q_{2 k-i}^{i},
$$

$\operatorname{com} Q_{2 k-i}^{i}= \pm M_{k k}$ (que é não-degenerado), chegamos em

$$
\mathcal{A}^{(i)^{T}} J \mathcal{A}^{(j)}=0
$$

para $k \leq i \leq 2 k-2$ e $0 \leq j \leq 2 k-i-2$. Isso prova nosso segundo resultado principal, dado pelo teorema abaixo

Teorema 16. Considerando o espaço $\left(\mathbb{R}^{2 k n}, \omega_{\text {can }}\right)$, a curva $p:[a, b] \rightarrow G r(n, 2 k n)$ definida na seção anterior satisfaz

- $j^{i} p:[a, b] \rightarrow \operatorname{Gr}((i+1) n, 2 k n)$ é uma curva de subespaços isotrópicos $i=0, \ldots, k-2$,

- $\ell=j^{k-1} p:[a, b] \rightarrow G r(k n, 2 k n)$ é uma curva de subespaços Lagrangeanos,

- $j^{i} p:[a, b] \rightarrow G r((i+1) n, 2 k n)$ é uma curva de subespaços coisotrópicos $i=k, \ldots, 2 k-1$.

\subsection{Casos Particulares}

Nesta seção daremos dois exemplos das contas acima nos casos $k=1$ e $k=2$

\subsubsection{Caso $k=1$}

Neste caso temos o funcional quadrático

$$
\mathcal{Q}(h)=\int_{a}^{b} \tilde{L}(t, h, \dot{h}) d t
$$

com

$$
\tilde{L}=\frac{1}{2}\left(h^{T} M_{00} h+\dot{h}^{T} M_{11} \dot{h}\right)+h^{T} M_{01} \dot{h}, \quad M_{00}=M_{00}^{T} \quad \text { e } \quad M_{11}=L_{11} .
$$

A equação de Jacobi neste caso fica

$$
\frac{\partial \tilde{L}}{\partial q_{0}}\left(j^{1} h(t)\right)-\frac{d}{d t}\left(\frac{\partial \tilde{L}}{\partial q_{1}}\left(j^{1} h(t)\right)\right)=0
$$

com as derivadas parciais dadas por

$$
\begin{aligned}
& \frac{\partial \tilde{L}}{\partial q_{0}}=M_{00} h+M_{01} \dot{h}, \\
& \frac{\partial \tilde{L}}{\partial q_{1}}=M_{11} \dot{h}+M_{01}^{T} h .
\end{aligned}
$$

Definição 16. Um ponto $t^{*} \in(a ; b]$ é dito um ponto conjugado à $a$ da equação (4.21) se existir solução não-trivial $h$ de $(4.21) \operatorname{com} h(a)=h\left(t^{*}\right)=0$.

A transformação de Legendre Leg $: \mathbb{R}^{2 n} \rightarrow \mathbb{R}^{2 n}$ aplicada à 1-jatos de curvas $h$ fica sendo $\operatorname{Leg}(h, \dot{h})=(y, z) \operatorname{com}$

$$
y=h \quad \text { e } \quad z=\frac{\partial \tilde{L}}{\partial q_{1}}=M_{11} \dot{h}+M_{01}^{T} h .
$$

Outra maneira de escrever

$$
\operatorname{Leg}(h, \dot{h})=\left(\begin{array}{l}
y \\
z
\end{array}\right)=\left(\begin{array}{cc}
\operatorname{Id}_{n} & 0 \\
M_{01} & M_{11}
\end{array}\right)\left(\begin{array}{l}
h \\
\dot{h}
\end{array}\right) .
$$


Pelo teorema 11 teremos que soluções $h$ de $(4.21)$ serão levadas em soluções $(y, z)=\operatorname{Leg}(h, \dot{h})$ do sistema abaixo, e vice-versa,

$$
\left(\begin{array}{c}
\dot{y} \\
\dot{z}
\end{array}\right)=\left(\begin{array}{cc}
-M_{11}^{-1} M_{01}^{T} & M_{11}^{-1} \\
M_{00}-M_{01} M_{11}^{-1} M_{01}^{T} & M_{01} M_{11}^{-1}
\end{array}\right)\left(\begin{array}{l}
y \\
z
\end{array}\right) .
$$

Este sistema é Hamiltoniano com

$$
A=-M_{11}^{-1} M_{01}^{T}, \quad B=M_{11}^{-1}, \quad C=M_{00}-M_{01} M_{11}^{-1} M_{01}^{T} .
$$

Considere um referencial da curva $p:[a, b] \rightarrow \operatorname{Gr}(n, 2 n)$ dado por

$$
\mathcal{A}=\left(\begin{array}{c}
h_{1}^{T} \\
h_{2}^{T} \\
\vdots \\
h_{2 n}^{T}
\end{array}\right)
$$

com $h_{i}$ sendo um conjunto fundamental de soluções de (4.21), e com as $n$ primeiras soluções $h_{i}$ se anulando em $t=a$.

Para cada solução $h_{i}$ considere imagem do 1-jato pela transformação de Legendre

$$
\operatorname{Leg}\left(h_{i}, \dot{h_{i}}\right)=\left(\begin{array}{c}
\mu_{i} \\
\zeta_{i}
\end{array}\right)=\left(\begin{array}{cc}
\operatorname{Id}_{n} & 0 \\
M_{01} & M_{11}
\end{array}\right)\left(\begin{array}{c}
h_{i} \\
\dot{h}_{i}
\end{array}\right)
$$

e construa a seguinte matriz

$$
\left(\begin{array}{ccc}
\mu_{1} & \cdots & \mu_{n} \\
\zeta_{1} & \cdots & \zeta_{n}
\end{array}\right)=\left(\begin{array}{cc}
\operatorname{Id}_{n} & 0 \\
M_{01} & M_{11}
\end{array}\right)\left(\begin{array}{ccc}
h_{1} & \cdots & h_{2 n} \\
\dot{h_{1}} & \cdots & \dot{h_{2 n}}
\end{array}\right)
$$

Com isso teremos que

$$
\left(\begin{array}{cc}
\mu_{1}^{T} & \zeta_{1}^{T} \\
\vdots & \vdots \\
\mu_{n}^{T} & \zeta_{n}^{T}
\end{array}\right)^{T}=(\mathcal{A} \mid \dot{\mathcal{A}})\left(\begin{array}{cc}
\operatorname{Id}_{n} & M_{01}^{T} \\
0 & M_{11}
\end{array}\right)=\left(\mathcal{A} \mid \mathcal{A} M_{01}^{T}+\dot{\mathcal{A}} M_{11}\right)
$$

Note que as linhas da matriz à esquerda acima satisfazem a equação (4.23), portanto

$$
\frac{d}{d t}\left(\mathcal{A} \mid \mathcal{A} M_{01}^{T}+\dot{\mathcal{A}} M_{11}\right)=\left(\mathcal{A} \mid \mathcal{A} M_{01}^{T}+\dot{\mathcal{A}} M_{11}\right)\left(\begin{array}{cc}
A^{T} & C \\
B & -A
\end{array}\right) .
$$

Supondo que em $t=a$ que a matriz $\left(\mathcal{A} \mid \mathcal{A} M_{01}^{T}+\dot{\mathcal{A}} M_{11}\right)(a)$ é simplética, e condizente com a condição inicial em $t=a$, por exemplo,

$$
\left(\mathcal{A} \mid \mathcal{A} M_{01}^{T}+\dot{\mathcal{A}} M_{11}\right)(a)=\left(\begin{array}{cc}
0 & \mathrm{Id}_{n} \\
-\mathrm{Id}_{n} & 0
\end{array}\right)
$$

teremos que $\left(\mathcal{A} \mid \mathcal{A} M_{01}^{T}+\dot{\mathcal{A}} M_{11}\right)$ será simplética para todo $t$, já que $\left(\begin{array}{cc}A^{T} & C \\ B & -A\end{array}\right)$ está na álgebra de 
Lie simplética. Logo, considerando $J$ a matriz da forma simplética canônica $\omega_{\text {can }}$ em $\mathbb{R}^{2 n}$, teremos

$$
\begin{gathered}
\left(\mathcal{A} \mid \mathcal{A} M_{01}^{T}+\dot{\mathcal{A}} M_{11}\right)^{T} J\left(\mathcal{A} \mid \mathcal{A} M_{01}^{T}+\dot{\mathcal{A}} M_{11}\right)=J \\
\left(\begin{array}{c}
\mathcal{A}^{T} \\
M_{01} \mathcal{A}^{T}+M_{11} \dot{\mathcal{A}}^{T}
\end{array}\right)\left(J \mathcal{A} \mid J \mathcal{A} M_{01}^{T}+J \dot{\mathcal{A}} M_{11}\right)=J \\
\left(\begin{array}{cc}
\mathcal{A}^{T} J \mathcal{A} & \mathcal{A}^{T} J \mathcal{A} M_{01}^{T}+\mathcal{A}^{T} J \dot{\mathcal{A}} M_{11} \\
M_{01} \mathcal{A}^{T} J \mathcal{A}+M_{11} \dot{\mathcal{A}}^{T} J \mathcal{A} & *
\end{array}\right)\left(\begin{array}{cc}
0 & \mathrm{Id}_{n} \\
-\mathrm{Id}_{n} & 0
\end{array}\right) .
\end{gathered}
$$

Então isso vai implicar que $\mathcal{A}^{T} J \mathcal{A}=0$, portanto $\ell=p:[a ; b] \rightarrow \operatorname{Gr}(n ; 2 n)$ será uma curva espalhante de subespaços Lagrangeanos.

\subsubsection{Caso $k=2$}

Neste caso teremos o funcional quadrático

$$
\mathcal{Q}(h)=\int_{a}^{b} \tilde{L}(t, h, \dot{h}, \ddot{h}) d t
$$

com

$\tilde{L}=\frac{1}{2}\left(h^{T} M_{00} h+\dot{h}^{T} M_{11} \dot{h}+\ddot{h}^{T} M_{22} \ddot{h}\right)+h^{T} M_{01} \dot{h}+\dot{h}^{T} M_{12} \ddot{h}, \quad M_{00}=M_{00}^{T}, M_{11}=M_{11}^{T}$, e $M_{22}=L_{22}$.

A equação de Jacobi fica

$$
\frac{\partial \tilde{L}}{\partial q_{0}}\left(j^{2} h(t)\right)-\frac{d}{d t}\left(\frac{\partial \tilde{L}}{\partial q_{1}}\left(j^{2} h(t)\right)\right)+\frac{d^{2}}{d t^{2}}\left(\frac{\partial \tilde{L}}{\partial q_{2}}\left(j^{2} h(t)\right)\right)=0,
$$

com as derivadas parciais dadas por

$$
\begin{gathered}
\frac{\partial \tilde{L}}{\partial q_{0}}=M_{00} h+M_{01} \dot{h}, \\
\frac{\partial \tilde{L}}{\partial q_{1}}=M_{11} \dot{h}+M_{01}^{T} h+M_{12} \ddot{h} \\
\frac{\partial \tilde{L}}{\partial q_{2}}=M_{22} \ddot{h}+M_{12}^{T} \dot{h}
\end{gathered}
$$

Definição 17. Um ponto $t^{*} \in(a ; b]$ é dito um ponto conjugado à $a$ da equação (4.24) se existir solução não-trivial $h$ de (4.24) $\operatorname{com} h(a)=h\left(t^{*}\right)=\dot{h}(a)=\dot{h}\left(t^{*}\right)=0$.

A transformação de Legendre Leg $: \mathbb{R}^{4 n} \rightarrow \mathbb{R}^{4 n}$ aplicada à 3-jatos de curvas $h$ fica sendo $\operatorname{Leg}\left(h, \dot{h}, \ddot{h}, h^{(3)}\right)=(y, z) \operatorname{com}$

$$
y=\left(\begin{array}{l}
h \\
\dot{h}
\end{array}\right) \quad \text { e } \quad z=\left(\begin{array}{c}
\frac{\partial \tilde{L}}{\partial q_{1}}-\frac{d}{d t}\left(\frac{\partial \tilde{L}}{\partial q_{2}}\right) \\
\frac{\partial \tilde{L}}{\partial q_{2}}
\end{array}\right)=\left(\begin{array}{c}
M_{11} \dot{h}+M_{01}^{T} h+M_{12} \ddot{h}-\frac{d}{d t}\left(M_{22} \ddot{h}+M_{12}^{T} \dot{h}\right) \\
M_{22} \ddot{h}+M_{12}^{T} \dot{h}
\end{array}\right) .
$$

Outra maneira de escrever

$$
\operatorname{Leg}\left(h, \dot{h}, \ddot{h}, h^{(3)}\right)=\left(\begin{array}{c}
y \\
z
\end{array}\right)=\left(\begin{array}{cccc}
\operatorname{Id}_{n} & 0 & 0 & 0 \\
0 & \operatorname{Id}_{n} & 0 & 0 \\
M_{01}^{T} & M_{11}-\dot{M}_{12}{ }^{T} & M_{12}-\dot{M}_{22}-M_{12}^{T} & -M_{22} \\
0 & M_{12}^{T} & M_{22} & 0
\end{array}\right)\left(\begin{array}{c}
h \\
\dot{h} \\
\ddot{h} \\
h^{(3)}
\end{array}\right) .
$$

Pelo teorema 11 teremos que soluções $h$ de (4.24) serão levadas em soluções $(y, z)=\operatorname{Leg}\left(h, \dot{h}, \ddot{h}, h^{(3)}\right)$ 
do sistema abaixo, e vice-versa,

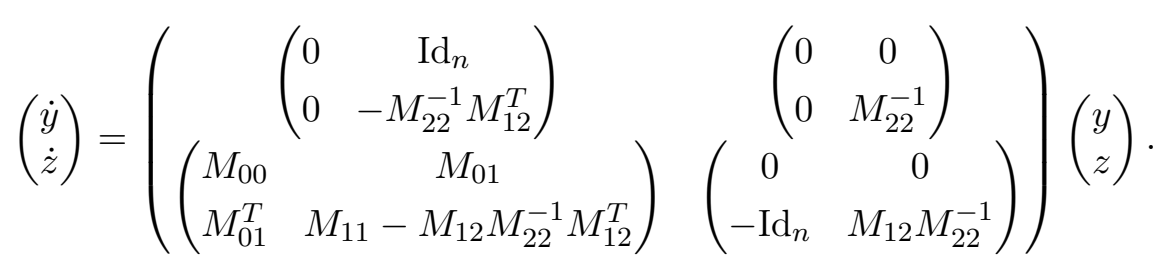

Este sistema é Hamiltoniano com

$$
A=\left(\begin{array}{cc}
0 & \mathrm{Id}_{n} \\
0 & -M_{22}^{-1} M_{12}^{T}
\end{array}\right), \quad B=\left(\begin{array}{cc}
0 & 0 \\
0 & M_{22}^{-1}
\end{array}\right), \quad C=\left(\begin{array}{cc}
M_{00} & M_{01} \\
M_{01}^{T} & M_{11}-M_{12} M_{22}^{-1} M_{12}^{T}
\end{array}\right) .
$$

Considere um referencial da curva $p:[a, b] \rightarrow \operatorname{Gr}(n, 4 n)$ dado por

$$
\mathcal{A}=\left(\begin{array}{c}
h_{1}^{T} \\
h_{2}^{T} \\
\vdots \\
h_{4 n}^{T}
\end{array}\right)
$$

com $h_{i}$ sendo um conjunto fundamental de soluções de (4.24), e as $2 n$ primeiras soluções $h_{i}$ se anulando até a primeira derivada em $t=a$.

Para cada solução $h_{i}$ considere sua imagem pela transformação de Legendre

$$
\operatorname{Leg}\left(h_{i}, \dot{h_{i}}, \ddot{h_{i}}, h_{i}^{(3)}\right)=\left(\begin{array}{c}
\mu_{i} \\
\zeta_{i}
\end{array}\right)=\left(\begin{array}{cccc}
\operatorname{Id}_{n} & 0 & 0 & 0 \\
0 & \operatorname{Id}_{n} & 0 & 0 \\
M_{01}^{T} & M_{11}-\dot{M}_{12}{ }^{T} & M_{12}-\dot{M}_{22}-M_{12}^{T} & -M_{22} \\
0 & M_{12}^{T} & M_{22} & 0
\end{array}\right)\left(\begin{array}{c}
h_{i} \\
\dot{h_{i}} \\
\ddot{h_{i}} \\
h_{i}^{(3)}
\end{array}\right) .
$$

Com isso teremos que

$$
\begin{aligned}
\left(\begin{array}{cc}
\mu_{1}^{T} & \zeta_{1}^{T} \\
\vdots & \vdots \\
\mu_{4 n}^{T} & \zeta_{4 n}^{T}
\end{array}\right) & =\left(\mathcal{A}|\dot{\mathcal{A}}| \ddot{\mathcal{A}} \mid \mathcal{A}^{(3)}\right)\left(\begin{array}{cccc}
\mathrm{Id} & 0 & M_{01} & 0 \\
0 & \text { Id } & M_{11}^{T}-M_{12} & M_{12} \\
0 & 0 & M_{12}^{T}-\dot{M}_{22}-M_{12} & M_{22} \\
0 & 0 & -M_{22} & 0
\end{array}\right)= \\
& =\left(\mathcal{A}|\dot{\mathcal{A}}| \mathcal{C}^{1} \mid \mathcal{C}^{2}\right),
\end{aligned}
$$

onde

$$
\begin{gathered}
\mathcal{C}^{1}=\mathcal{A} M_{01}+\dot{\mathcal{A}}\left(M_{11}^{T}-\dot{M}_{12}\right)+\ddot{\mathcal{A}}\left(M_{12}^{T}-\dot{M}_{22}-M_{12}\right)+\mathcal{A}^{(3)}\left(-M_{22}\right), \mathrm{e} \\
\mathcal{C}^{2}=\dot{\mathcal{A}} M_{12}+\ddot{\mathcal{A}} M_{22}
\end{gathered}
$$

Note que as linhas da matriz à esquerda acima satisfazem a equação (4.26), portanto

$$
\frac{d}{d t}\left(\mathcal{A}|\dot{\mathcal{A}}| \mathcal{C}^{1} \mid \mathcal{C}^{2}\right)=\left(\mathcal{A}|\dot{\mathcal{A}}| \mathcal{C}^{1} \mid \mathcal{C}^{2}\right)\left(\begin{array}{cc}
A^{T} & C \\
B & -A
\end{array}\right)
$$

Supondo que em $t=a$ a matriz $\left(\mathcal{A}|\dot{\mathcal{A}}| \mathcal{C}^{1} \mid \mathcal{C}^{2}\right)(a)$ é simplética, e condizente com a condição inicial em $t=a$, por exemplo, por exemplo,

$$
\left(\mathcal{A}|\dot{\mathcal{A}}| \mathcal{C}^{1} \mid \mathcal{C}^{2}\right)(a)=\left(\begin{array}{cccc}
0 & 0 & 0 & \operatorname{Id}_{n} \\
0 & 0 & \operatorname{Id}_{n} & 0 \\
0 & -\mathrm{Id}_{n} & 0 & 0 \\
-\mathrm{Id}_{n} & 0 & 0 & 0
\end{array}\right)
$$


teremos que $\left(\mathcal{A}|\dot{\mathcal{A}}| \mathcal{C}^{1} \mid \mathcal{C}^{2}\right)$ será simplética para todo $t$, já que $\left(\begin{array}{cc}A^{T} & C \\ B & -A\end{array}\right)$ está na álgebra de Lie simplética. Logo considerando $J$ a matriz da forma simplética canônica $\omega_{\text {can }}$ em $\mathbb{R}^{4 n}$ teremos

$$
\begin{aligned}
& \left(\mathcal{A}|\dot{\mathcal{A}}| \mathcal{C}^{1} \mid \mathcal{C}^{2}\right)^{T} J\left(\mathcal{A}|\dot{\mathcal{A}}| \mathcal{C}^{1} \mid \mathcal{C}^{2}\right)=J \\
& \left(\begin{array}{cccc}
\mathcal{A}^{T} J \mathcal{A} & \mathcal{A}^{T} J \dot{\mathcal{A}} & \mathcal{A}^{T} J \mathcal{C}^{1} & \mathcal{A}^{T} J \mathcal{C}^{2} \\
\dot{\mathcal{A}}^{T} J \mathcal{A} & \dot{\mathcal{A}}^{T} J \dot{\mathcal{A}} & \dot{\mathcal{A}}^{T} J \mathcal{C}^{1} & \dot{\mathcal{A}}^{T} J \mathcal{C}^{2} \\
\mathcal{C}^{1^{T} J \mathcal{A}} & \mathcal{C}^{1^{T} J \dot{\mathcal{A}}} & \mathcal{C}^{1^{T}} J \mathcal{C}^{1} & \mathcal{C}^{1^{T} J \mathcal{C}^{2}} \\
\mathcal{C}^{2^{T}} J \mathcal{A} & \mathcal{C}^{2^{T}} J \dot{\mathcal{A}} & \mathcal{C}^{2^{T}} J \mathcal{C}^{1} & \mathcal{C}^{2^{T}} J \mathcal{C}^{2}
\end{array}\right)=\left(\begin{array}{cccc}
0 & 0 & \mathrm{Id}_{n} & 0 \\
0 & 0 & 0 & \mathrm{Id}_{n} \\
-\operatorname{Id}_{n} & 0 & 0 & 0 \\
0 & -\mathrm{Id}_{n} & 0 & 0 .
\end{array}\right)
\end{aligned}
$$

A equação acima implica que

$$
\mathcal{A}^{T} J \mathcal{A}=\mathcal{A}^{T} J \dot{\mathcal{A}}=\dot{\mathcal{A}}^{T} J \dot{\mathcal{A}}=\mathcal{C}^{2^{T}} J \mathcal{A}=0 .
$$

Desenvolvendo a última igualdade teremos

$$
\mathcal{C}^{2^{T}} J \mathcal{A}=0 \Rightarrow\left(\dot{\mathcal{A}} M_{12}+\ddot{\mathcal{A}} M_{22}\right)^{T} J \mathcal{A}=0 \Rightarrow M_{22} \ddot{\mathcal{A}}^{T} J \mathcal{A}=0 \Rightarrow \ddot{\mathcal{A}}^{T} J \mathcal{A}=0 .
$$

Portanto

- $p:[a, b] \rightarrow \operatorname{Gr}(n, 4 n)$ é curva de subespaços isotrópicos,

- $\ell=j^{1} p:[a, b] \rightarrow \operatorname{Gr}(2 n, 4 n)$ é curva de subespaços Lagrangeanos, e

- $j^{2} p:[a, b] \rightarrow \operatorname{Gr}(3 n, 4 n)$ é curva de subespaços coisotrópicos. 


\section{Capítulo 5}

\section{Problema Inverso Linear}

O problema inverso (clássico) do cálculo das variações pode ser enunciado da seguinte forma: dado um sistema de equações diferenciais de ordem 2 quando existe um Lagrangeano $L$ tal que o sistema original é dado pelas equações de Euler-Lagrange de $L$ ? Este problema foi estudado inicialmente por Helmholtz em 1887. No trabalho de Douglas ([Dou41]) são apresentadas e desenvolvidas tais condições necessárias e suficientes, que levam o nome de condições de Helmholtz. Na linha deste estudo, Henneaux ([Hen82]) e Sarlet-Engels-Bahar ([SEB82]) melhoraram estas condições de modo a torná-las mais "calculáveis".

A partir desses trabalhos, seguindo a linha de linearização de projetivização, usando o conceito de curvas espalhantes em [ÁPD09] (veja seção 1.2) e o desenvolvimento de Sarlet-Engels-Bahar, nós encontramos condições necessárias e suficientes para tais curvas provirem de um problema variacional. Um resultado interessante que obtivemos é que a propriedade de vir de um problema variacional apenas irá depender da curva projetiva associada à equação diferencial.

\subsection{Condições de Helmholtz}

Considere o seguinte sistema de ordem 2

$$
\ddot{x}+2 A(t) \dot{x}+B(t) x=0,
$$

onde $A(t)$ e $B(t)$ são curvas de matrizes $n \times n$. As condições necessárias e suficientes para que (5.1) seja dada pelas equações de Euler-Lagrange de algum $L: \mathbb{R}^{n} \times \mathbb{R}^{n} \rightarrow \mathbb{R}$ são conhecidas como condições de Helmholtz, e são elas: existir uma curva $Z(t)$ de matrizes $n \times n$ (chamado de matriz múltipla) tal que $Z(t)$ seja invertível para todo $t$ e satisfaça as seguintes condições

$$
\begin{gathered}
Z_{0}=Z_{0}^{T} \\
\dot{Z}=A^{T} Z+Z A, \\
Z \Phi^{(0)}=\left(Z \Phi^{(0)}\right)^{T}
\end{gathered}
$$

onde $Z_{0}=Z\left(t_{0}\right)$ para algum $t_{0}$ e $\Phi^{(0)}(t)=B(t)-A(t)^{2}-\dot{A}(t)$. Note que tal $Z(t)$ será simétrica para todo $t$ : a função $V=Z-Z^{T}$ vai satisfazer a equação diferencial linear $\dot{V}=A^{T} V+V A$ com condição inicial $V\left(t_{0}\right)=0$.

\subsection{Referenciais Normais e Schwarziana}

Considere $\ell(t) \in \operatorname{Gr}(n, 2 n)$ uma curva espalhante e seja um referencial $\mathcal{A}$ de $\ell$. Teremos que o referencial $\mathcal{A}$ vai satisfazer a seguinte relação diferencial

$$
\ddot{\mathcal{A}}+2 \dot{\mathcal{A}} P(t)+\mathcal{A} Q(t)=0 .
$$


Neste caso as $2 n$ linhas de $\mathcal{A}$ irão satisfazer a seguinte equação diferencial em $x=x(t) \in \mathbb{R}^{n}$ :

$$
\ddot{x}+2 P(t)^{T} \dot{x}+Q(t)^{T} x=0 .
$$

Os seguintes lemas indicam o que acontece com a equação diferencial quando mudamos de referencial e quando temos referenciais congruentes:

Lema 6. Seja $\tilde{\mathcal{A}}$ outro referencial de $\ell$ que satisfaz $\tilde{\mathcal{A}}(t)=\mathcal{A}(t) X(t)$ com $X(t)$ matriz $n \times n$ invertivel para todo t. Então $\tilde{\mathcal{A}}(t)$ irá satisfazer

$$
\ddot{\widetilde{\mathcal{A}}}+2 \dot{\tilde{\mathcal{A}}} \tilde{P}(t)+\tilde{\mathcal{A}} \tilde{Q}(t)=0,
$$

com $\tilde{P}$ e $\tilde{Q}$ dados por

$$
\begin{gathered}
\tilde{P}=X^{-1} P X-X^{-1} \dot{X} \\
\tilde{Q}=X^{-1} Q X-X^{-1} \ddot{X}-2 X^{-1} \dot{X} \tilde{P} .
\end{gathered}
$$

Ainda mais, as equações diferenciais relacionadas aos referencias $\mathcal{A}$ e $\tilde{\mathcal{A}}$ ficam sendo respectivamente

$$
\begin{aligned}
& \ddot{x}+2 P(t)^{T} \dot{x}+Q(t)^{T} x=0 \\
& \ddot{\tilde{x}}+2 \tilde{P}(t)^{T} \dot{\tilde{x}}+\tilde{Q}(t)^{T} \tilde{x}=0,
\end{aligned}
$$

e teremos que $x(t)$ é solução de (5.4) se e somente se $\tilde{x}(t)=(X(t))^{T} x(t)$ for solução de (5.5).

Lema 7. Seja $\mathcal{B}(t)$ um referencial congruente à $\mathcal{A}(t)$, isto é, $\mathcal{B}(t)=\mathbf{T} \mathcal{A}(t)$ com $\mathbf{T}$ matriz $2 n \times 2 n$ invertivel. Então $\mathcal{B}(t)$ irá satisfazer

$$
\ddot{\mathcal{B}}+2 \dot{\mathcal{B}} P(t)+\mathcal{B} Q(t)=0,
$$

e a equação diferencial relacionada à $\mathcal{B}$ será igual à equação diferencial relacionada à $\mathcal{A}$.

Resolver o problema inverso do cálculo das variações para o referencial $\mathcal{A}$ neste caso seria encontrar um múltiplo $Z(t)$ tal que a equação diferencial

$$
Z(t) \ddot{x}+2 Z(t) P(t)^{T} \dot{x}+Z(t) Q(t)^{T} x=0
$$

seja a equação de Euler-Lagrange para algum Lagrangeano $L$.

A Schwarziana do referencial $\mathcal{A}$ acima (veja 1.3) é dada por

$$
S_{\mathcal{A}}(t)=Q(t)-P(t)^{2}-\dot{P}(t)
$$

e note que com a definição de $\Phi^{(0)}$ acima, para este referencial teremos

$$
\Phi^{(0)}(t)=Q(t)^{T}-\left(P(t)^{2}\right)^{T}-\dot{P}(t)^{T}=S_{\mathcal{A}}(t)^{T} .
$$

Note também que pelo teorema 1 podemos associar cada curva espalhante à uma curva de transformações lineares $S_{\ell}: I \rightarrow \operatorname{Lin}(\ell(t) ; \ell(t))$.

Um referencial normal $\mathcal{B}$ de $\ell$ é um referencial de $\ell$ tal que as colunas de $\ddot{\mathcal{B}}$ são combinações lineares apenas das colunas de $\mathcal{B}$ (veja definição 5 ). Através de (5.3) podemos construir um referencial normal $\mathcal{B}$ a partir de qualquer referencial $\mathcal{A}$ de $\ell$. Para isso considere $X$ satisfazendo a seguinte equação diferencial linear de primeira ordem

$$
\dot{X}=P(t) X, \quad X\left(t_{0}\right)=\mathrm{Id} .
$$


Com isso $\tilde{P}$ e $\tilde{Q}$ serão dadas por

$$
\begin{gathered}
\tilde{P}=0 \\
\tilde{Q}=X^{-1} Q X-X^{-1} \ddot{X}=X^{-1} Q X-X^{-1} \dot{P} X-X^{-1} P^{2} X=X^{-1} S_{\mathcal{A}} X=S_{\mathcal{B}} .
\end{gathered}
$$

E o referencial $\mathcal{B}=\mathcal{A} X(t)$ irá satisfazer

$$
\ddot{\mathcal{B}}+\mathcal{B} S_{\mathcal{B}}(t)=0
$$

com equação diferencial associada

$$
\ddot{\tilde{x}}+S_{\mathcal{B}}(t)^{T} \tilde{x}=0 .
$$

Isso prova parte da proposição 3, e teremos o seguinte resultado:

Teorema 17. O sistema (5.2) virá de um problema variacional se e somente se o sistema (5.8) vir de um problema variacional.

Demonstração. Observe que o sistema (5.2) virá de um problema variacional se e somente se existir um múltiplo $Z=Z(t)$ com

$$
\begin{gathered}
Z_{0}=Z_{0}^{T} \\
\dot{Z}=P Z+Z P^{T} \\
Z(t) S_{\mathcal{A}}(t)^{T}=S_{\mathcal{A}}(t) Z(t),
\end{gathered}
$$

onde $Z_{0}=Z\left(t_{0}\right)$.

Da mesma forma, o sistema (5.8) virá de um problema variacional se existir um múltiplo constante $Z_{0}$ tal que

$$
\begin{gathered}
Z_{0}=Z_{0}^{T} \\
Z_{0} S_{\mathcal{B}}(t)^{T}=S_{\mathcal{B}}(t) Z_{0} .
\end{gathered}
$$

Suponha que (5.9) vale. Então o múltiplo $Z$ neste caso vai ser dado por

$$
Z=X Z_{0} X^{T}
$$

com $X$ satisfazendo (5.6). De fato, calculando a derivada de $Z$

$$
\dot{Z}=\dot{X} Z_{0} X^{T}+X Z_{0} \dot{X}^{T}=P X Z_{0} X^{T}+X Z_{0}(P X)^{T}=P Z+Z P^{T},
$$

e como para este $Z$ temos $Z\left(t_{0}\right)=Z_{0}$ (pois $X\left(t_{0}\right)=$ Id), pelo teorema de unicidade de E.D.O.s teremos que $Z$ vai ser dada pela igualdade acima. Ainda pelo fato de $Z$ satisfazer (5.9) teremos

$$
\begin{gathered}
X Z_{0} X^{T} S_{\mathcal{A}}(t)^{T}=S_{\mathcal{A}}(t) X Z_{0} X^{T} \\
Z_{0} X^{T} S_{\mathcal{A}}(t)^{T} X^{-1^{T}}=X^{-1} S_{\mathcal{A}}(t) X Z_{0} \\
Z_{0} S_{\mathcal{B}}(t)^{T}=S_{\mathcal{B}}(t) Z_{0},
\end{gathered}
$$

logo $Z_{0}$ será múltiplo do sistema (5.8).

Reciprocamente, se (5.10) for satisfeita então tome $Z$ como sendo $Z=X Z_{0} X^{T}$. E, da mesma forma que acima, teremos que $Z$ irá satisfazer (5.9), e então (5.2) virá de um problema variacional.

Um resultado novo que obtivemos, dado pelo teorema a seguir, é o seguinte: se algum referencial $\mathcal{A}$ de $\ell$ vier de um problema variacional então qualquer outro referencial $\tilde{\mathcal{A}}$ de $\ell$ também virá de um problema variacional. Isto é, a propriedade de vir de um problema variacional depende apenas de curva espalhante $\ell$.

Teorema 18. Sejam $\mathcal{A}$ e $\tilde{\mathcal{A}}$ dois referenciais da curva $\ell$. Então $\mathcal{A}$ virá de um problema variacional se e somente se $\widetilde{\mathcal{A}}$ vir de um problema variacional. 
Demonstração. Tome referenciais normais $\mathcal{B}$ e $\tilde{\mathcal{B}}$ de $\ell$ construídos como acima a partir de $\mathcal{A}$ e $\tilde{\mathcal{A}}$, respectivamente (veja proposição 3). Assim pelo teorema acima o problema ficará reduzido em mostrar que $\mathcal{B}$ vem de um problema variacional se, e somente se, $\tilde{\mathcal{B}}$ vem de um problema variacional.

Sabemos da proposição 3 que existe uma matriz invertível fixa $X_{0} n \times n$ tal que $\tilde{\mathcal{B}}(t)=\mathcal{B}(t) X_{0}$ para todo $t$, e com isso $S_{\tilde{\mathcal{B}}}=X_{0}^{-1} S_{\mathcal{B}} X_{0}$.

Supondo que o referencial $\mathcal{B}$ admita um múltiplo (constante) $Z_{0}$ teremos

$$
\begin{gathered}
Z_{0}=Z_{0}^{T} \\
Z_{0} S_{\mathcal{B}}(t)^{T}=S_{\mathcal{B}}(t) Z_{0} .
\end{gathered}
$$

Logo teremos

$$
\begin{gathered}
Z_{0}\left(X_{0} S_{\tilde{\mathcal{B}}} X_{0}^{-1}\right)^{T}=\left(X_{0} S_{\tilde{\mathcal{B}}} X_{0}^{-1}\right) Z_{0} \\
Z_{0}\left(X_{0}^{-1}\right)^{T} S_{\tilde{\mathcal{B}}}^{T} X_{0}^{T}=X_{0} S_{\tilde{\mathcal{B}}} X_{0}^{-1} Z_{0} \\
\left(X_{0}^{-1} Z_{0}\left(X_{0}^{-1}\right)^{T}\right) S_{\tilde{\mathcal{B}}}^{T}=S_{\tilde{\mathcal{B}}}\left(X_{0}^{-1} Z_{0}\left(X_{0}^{-1}\right)^{T}\right),
\end{gathered}
$$

e então $\tilde{\mathcal{B}}$ terá um múltiplo $\tilde{Z}_{0}=X_{0}^{-1} Z_{0}\left(X_{0}^{-1}\right)^{T}$, ou seja, $\tilde{\mathcal{B}}$ virá de um problema variacional.

A recíproca é inteiramente análoga.

Isso nos leva à seguinte definição natural:

Definição 18. Uma curva espalhante $\ell: I \rightarrow \operatorname{Gr}(n ; 2 n)$ é dita variacional (Jacobi) se para algum referencial $\mathcal{A}$ tivermos que a equação diferencial associada à $\mathcal{A}$ vem de um problema variacional.

Para um referencial normal $\mathcal{B}$ de $\ell$ trocaremos (5.10) por uma condição equivalente

$$
\begin{gathered}
W_{0}=W_{0}^{T} \\
S_{\mathcal{B}}(t)^{T} W_{0}=W_{0} S_{\mathcal{B}}(t),
\end{gathered}
$$

onde $W_{0}=Z_{0}^{-1}$. Note que com isso se, $\tilde{\mathcal{B}}(t)=\mathcal{B}(t) X_{0}$, então pelas contas acima o múltiplo de $\tilde{\mathcal{B}}$ será $\tilde{Z}_{0}=X_{0}^{-1} Z_{0}\left(X_{0}^{-1}\right)^{T}$, ou $\tilde{W}_{0}=\left(\tilde{Z}_{0}\right)^{-1}=X_{0}^{T} W_{0} X_{0}$. Note também que, pela equação (5.11), o múltiplo do referencial normal $\mathcal{B}=\mathcal{A} X(t)$ construído a partir de $\mathcal{A}$ será dado por $Z_{0}=$ $X^{-1} Z(t)\left(X^{-1}\right)^{T}$ com $X$ satisfazendo (5.6) ou, escrevendo de outra forma, $W_{0}=X^{T} W(t) X$ com $W(t)=(Z(t))^{-1}$. Uma condição que o múltiplo (inverso) $W(t)$ relacionado ao referencial $\mathcal{A}$ deverá satisfazer é

$$
S_{\mathcal{A}}(t)^{T} W(t)=W(t) S_{\mathcal{A}}(t) .
$$

Com as considerações acima, se tivermos dois referenciais arbitrários de uma curva espalhante variacional $\ell, \tilde{\mathcal{A}}$ e $\mathcal{A}$, relacionados por $\tilde{\mathcal{A}}=\mathcal{A} X(t)$, com $X(t)$ invertível para todo $t$, então os múltiplos (inversas) $\tilde{W}(t)$ e $W(t)$ estarão relacionados por:

$$
\tilde{W}(t)=X(t)^{T} W(t) X(t)
$$

para todo $t$. Logo para uma curva espalhante variacional $\ell$ fica bem definido uma curva de formas bilineares simétricas $W_{\ell}: I \rightarrow \mathrm{B}_{\mathrm{sym}}(\ell(t))$.

Fica claro agora o porquê de escrever a condição ter múltiplo utilizando a inversa: ao trocar de referencial notamos uma transformação do tipo de formas bilineares, e também a relação abaixo

$$
S_{\mathcal{A}}(t)^{T} W(t)=W(t) S_{\mathcal{A}}(t)
$$

é independe do referencial escolhido. Logo para uma curva espalhante variacional $\ell$ fica bem definido uma curva de formas bilineares simétricas $W_{\ell}: I \rightarrow \mathrm{B}_{\text {sym }}(\ell(t))$. E a condição acima fica traduzido que a Schwarziana $S_{\ell}$ deve ter auto-adjunta com relação à $W_{\ell}$ para todo $t$, isto é,

$$
W_{\ell(t)}\left(S_{\ell(t)} v_{1}(t), v_{2}(t)\right)=W_{\ell(t)}\left(v_{1}(t), S_{\ell(t)} v_{2}(t)\right)
$$


para todo $t$ e para todo $v_{1}(t), v_{2}(t) \in \ell(t)$.

Se $\mathcal{B}$ é um referencial normal da curva espalhante variacional $\ell$ então uma função Lagrangeana $L$ para o sistema

$$
Z_{0} \ddot{\tilde{x}}+Z_{0} S_{\mathcal{B}}^{T} \tilde{x}=0
$$

onde $Z_{0}$ é múltiplo de $\mathcal{B}$, será dada por

$$
L=\frac{1}{2}\left(\dot{\tilde{x}}^{T} Z_{0} \dot{\tilde{x}}-\tilde{x}^{T} Z_{0} S_{\mathcal{B}}^{T} \tilde{x}\right)
$$

Caso $\mathcal{A}$ seja um referencial arbitrário de $\ell$ então a função Lagrangeana fica escrita como

$$
L=\frac{1}{2} \dot{x}^{T} Z \dot{x}+x^{T} P Z \dot{x}+\frac{1}{2} x^{T}\left(P Z P^{T}-Z S_{\mathcal{A}}^{T}\right) x,
$$

usando que $\tilde{x}=X(t) x, Z=X Z_{0} X^{T}$ e $\dot{X}=P X$.

\subsection{Wronskiano e Curva de Lagrangeanos}

Uma outra forma equivalente que encontramos de dizer se uma curva espalhante é variacional é dada pelo seguinte teorema

Teorema 19. Seja $\ell$ uma curva espalhante. Então $\ell$ é variacional se e somente se $\ell$ é uma curva de Lagrangeanos para alguma forma simplética $\omega$.

Para a prova deste teorema precisamos do conceito de Wronskiano para curvas de Lagrangeanos encontrado em Alvarez-Durán ([ÁPD09]).

Seja $\left(\mathbb{R}^{2 n}, \omega\right)$ onde $\omega$ é uma forma simplética em $\mathbb{R}^{2 n}$. O conjunto $\Lambda_{n}$ é o conjunto de todos os subespaços Lagrangeanos de $\left(\mathbb{R}^{2 n}, \omega\right)$.

Se $\ell: I \rightarrow \Lambda_{n}$ é uma curva de subespaços Lagrangeanos então dizemos que $\mathcal{A}: I \rightarrow \mathbb{R}^{2 n \times n}$ é uma curva de referenciais Lagrangeanos se para todo $t \in I$ as colunas de $\mathcal{A}(t)$ gerarem $\ell(t)$.

Denotando também por $\omega$ a matriz $2 n \times 2 n$ de $\omega$ em alguma base $\beta$ de $\mathbb{R}^{2 n}$, a condição de $\mathcal{A}$ ser uma curva de referencias Lagrangeanos se exprime em

$$
\mathcal{A}(t)^{T} \omega \mathcal{A}(t)=0 \quad \text { e } \quad \operatorname{posto}(\mathcal{A}(t))=n
$$

para todo $t \in I$. Derivando a primeira dessas condições temos

$$
\dot{\mathcal{A}}(t)^{T} \omega \mathcal{A}(t)+\mathcal{A}(t)^{T} \omega \dot{\mathcal{A}}(t)=0 .
$$

Definição 19. O Wronskiano da curva de referenciais Lagrangeanos $\mathcal{A}(t)$ é a curva de matrizes $n \times n$ definida por $W(t)=-\mathcal{A}(t)^{T} \omega \dot{\mathcal{A}}(t)$.

Proposição 8 (Álvarez-Durán (2009) [ÁPD09]). O Wronskiano $W(t)$ da curva de referenciais Lagrangeanos $\mathcal{A}(t)$ satisfaz as seguintes propriedades

- $W(t)$ é simétrica para todo $t$.

- Se $X(t)$ é uma curva de matrizes $n \times n$ invertiveis, então o Wronskiano da curva de referenciais Lagrangeanos $\mathcal{A}(t) X(t)$ é dado por $X(t)^{T} W(t) X(t)$.

- Se $\mathbf{S}$ é um simplectomorfismo linear de $\left(\mathbb{R}^{2 n}, \omega\right)$, o Wronskiano de $\mathbf{S} \mathcal{A}(t)$ é também $W(t)$.

- A curva de referenciais Lagrangeanos $\mathcal{A}(t)$ é espalhante se, e somente se, $W(t)$ é invertivel para todo t.

Note pela proposição anterior que para uma curva espalhante de Lagrangeanos $\ell$ vai ficar bem definida uma forma bilinear simétrica $W_{\ell}: I \rightarrow \mathrm{B}_{\mathrm{sym}}(\ell(t))$. 
Para curvas de Jacobi o Wronskiano será essencialmente a "métrica" do problema variacional. Considere um referencial $\mathcal{A}$ de $\ell$ formado por linhas que são soluções l.i. da equação abaixo

$$
\frac{d}{d t}(P(t) \dot{x})-Q(t) x=0,
$$

com $P(t)$ e $Q(t)$ simétricas e $P(t)$ não-degenerada para todo $t$.

Note que a equação acima é a equação de Euler-Lagrange do funcional quadrático

$$
\int_{a}^{b}\langle P \dot{x}, \dot{x}\rangle+\langle Q x, x\rangle d t
$$

e teremos

Proposição 9 (Álvarez-Durán (2009) [ÁPD09]). Nas condições do parágrafo anterior, se a matriz $(\mathcal{A}(a) \mid \dot{\mathcal{A}}(a) P(a))$ na condição inicial $t=$ a for simplética, então $\mathcal{A}$ é um referencial de subespaços Lagrangeanos tal que o Wronskiano deste referencial é $W(t)=P(t)^{-1}$.

Supondo que o funcional acima é, a Hessiana de um certo funcional com Lagrangeano ("métrica") $L$, como desenvolvido em 1.1, teremos que a matriz $P(t)$ corresponderá à Hessiana de $L$ como relação às velocidades. Disto seguirá que, por exemplo, no caso que de métricas $L$ semi-Riemannianas, a assinatura de $L$ será a mesma assinatura do Wronskiano obtido pela forma simplética pela transformação de Legendre associada à $L$.

Com a proposição 8 podemos demonstrar o teorema anterior:

Demonstração do Teorema 19. $(\Rightarrow)$ Suponha que $\ell$ seja variacional. Seja então $\mathcal{B}$ um referencial normal e $W_{0}$ um múltiplo com relação à este referencial. Como $W_{0}$ é simétrica e não-degenerada, existe uma matriz $X_{0}$ invertível fixa tal que

$$
X_{0}^{T} W_{0} X_{0}=I_{n, k}=\left(\begin{array}{cc}
-I_{k} & 0 \\
0 & I_{n-k}
\end{array}\right),
$$

onde $I_{j}$ é a matriz identidade $j \times j$. Então, sem perda de generalidade, podemos supor $W_{0}=I_{n, k}$ já que a condição de múltiplo para referenciais normais é invariante por mudanças da forma acima. Note que neste caso teremos $W_{0}^{-1}=W_{0}$ e $W_{0}^{2}=I_{n}$.

Considere a base $\beta$ de $\mathbb{R}^{n}$ formada pelas colunas de $\left(\mathcal{B}\left(t_{0}\right) \mid \dot{\mathcal{B}}\left(t_{0}\right) W_{0}\right)$. Defina uma forma simplética $\omega$ tal que a base $\beta$ seja simplética com relação à $\omega$ ou, de outro modo, tal que a matriz de $\omega$ na base $\beta$ seja dada por

$$
\left(\begin{array}{cc}
0 & -I_{n} \\
I_{n} & 0
\end{array}\right)
$$

Como $\mathcal{B}$ é normal temos $\ddot{\mathcal{B}}(t)+\mathcal{B}(t) S_{\mathcal{B}}(t)=0$, e então temos a identidade matricial

$$
\frac{d}{d t}\left(\mathcal{B}(t) \mid \dot{\mathcal{B}}(t) W_{0}\right)=\left(\mathcal{B}(t) \mid \dot{\mathcal{B}}(t) W_{0}\right)\left(\begin{array}{cc}
0 & -S_{\mathcal{B}}(t) W_{0} \\
W_{0} & 0
\end{array}\right) .
$$

Pelo fato que $S_{\mathcal{B}}^{T} W_{0}=W_{0} S_{\mathcal{B}}$ ou, equivalentemente, $W_{0} S_{\mathcal{B}}^{T}=S_{\mathcal{B}} W_{0}$, teremos que

$$
\begin{gathered}
\omega\left(\begin{array}{cc}
0 & -S_{\mathcal{B}}(t) W_{0} \\
W_{0} & 0
\end{array}\right)+\left(\begin{array}{cc}
0 & -S_{\mathcal{B}}(t) W_{0} \\
W_{0} & 0
\end{array}\right)^{T} \omega= \\
=\left(\begin{array}{cc}
0 & -I_{n} \\
I_{n} & 0
\end{array}\right)\left(\begin{array}{cc}
0 & -S_{\mathcal{B}}(t) W_{0} \\
W_{0} & 0
\end{array}\right)+\left(\begin{array}{cc}
0 & -S_{\mathcal{B}}(t) W_{0} \\
W_{0} & 0
\end{array}\right)^{T}\left(\begin{array}{cc}
0 & -I_{n} \\
I_{n} & 0
\end{array}\right)=0 .
\end{gathered}
$$

Logo a curva de matrizes acima estará na álgebra de Lie simplética, isto é,

$$
\left(\begin{array}{cc}
0 & -S_{\mathcal{B}}(t) W_{0} \\
W_{0} & 0
\end{array}\right) \in \operatorname{sp}(2 n)
$$


Pela identidade e observação acima teremos

$$
\frac{d}{d t}\left(\mathcal{B}(t) \mid \dot{\mathcal{B}}(t) W_{0}\right)\left(\mathcal{B}(t) \mid \dot{\mathcal{B}}(t) W_{0}\right)^{-1}=\left(\mathcal{B}(t) \mid \dot{\mathcal{B}}(t) W_{0}\right)\left(\begin{array}{cc}
0 & -S_{\mathcal{B}}(t) W_{0} \\
W_{0} & 0
\end{array}\right)\left(\mathcal{B}(t) \mid \dot{\mathcal{B}}(t) W_{0}\right)^{-1} \in \operatorname{sp}(2 n)
$$

Logo

$$
\begin{gathered}
\omega\left(\frac{d}{d t}\left(\mathcal{B}(t) \mid \dot{\mathcal{B}}(t) W_{0}\right)\left(\mathcal{B}(t) \mid \dot{\mathcal{B}}(t) W_{0}\right)^{-1}\right)+\left(\frac{d}{d t}\left(\mathcal{B}(t) \mid \dot{\mathcal{B}}(t) W_{0}\right)\left(\mathcal{B}(t) \mid \dot{\mathcal{B}}(t) W_{0}\right)^{-1}\right)^{T} \omega=0 \\
\left(\mathcal{B}(t) \mid \dot{\mathcal{B}}(t) W_{0}\right)^{T} \omega\left(\frac{d}{d t}\left(\mathcal{B}(t) \mid \dot{\mathcal{B}}(t) W_{0}\right)\right)+\left(\frac{d}{d t}\left(\mathcal{B}(t) \mid \dot{\mathcal{B}}(t) W_{0}\right)\right)^{T} \omega\left(\mathcal{B}(t) \mid \dot{\mathcal{B}}(t) W_{0}\right)=0 \\
\frac{d}{d t}\left(\left(\mathcal{B}(t) \mid \dot{\mathcal{B}}(t) W_{0}\right)^{T} \omega\left(\mathcal{B}(t) \mid \dot{\mathcal{B}}(t) W_{0}\right)\right)=0 .
\end{gathered}
$$

Como a derivada da matriz acima é zero, e em $t_{0}$ temos

$$
\left(\mathcal{B}\left(t_{0}\right) \mid \mathcal{B}\left(t_{0}\right) W_{0}\right)^{T} \omega\left(\mathcal{B}\left(t_{0}\right) \mid \mathcal{B}\left(t_{0}\right) W_{0}\right)=\omega
$$

chegamos que

$$
\left(\mathcal{B}(t) \mid \mathcal{B}(t) W_{0}\right)^{T} \omega\left(\mathcal{B}(t) \mid \mathcal{B}(t) W_{0}\right)=\omega
$$

para todo $t \in I$. $\operatorname{Logo}\left(\mathcal{B}(t) \mid \mathcal{B}(t) W_{0}\right)$ é simplética para todo $t$ e em particular $\mathcal{B}(t)^{T} \omega \mathcal{B}(t)=0$. Logo $\mathcal{B}(t)$ é uma curva de referenciais Lagrangeanos para $\omega$, e então $\ell$ é curva de Lagrangeanos.

$(\Leftarrow)$ Se a curva espalhante $\ell$ é uma curva de subespaços Lagrangeanos para alguma forma $\omega$, escolha uma base $\beta$ de $\mathbb{R}^{2 n}$ tal que $\omega$ nesta base $\beta$ se escreve como

$$
\omega=\left(\begin{array}{cc}
0 & -I_{n} \\
I_{n} & 0
\end{array}\right)
$$

Seja $\mathcal{B}$ um referencial normal de $\ell$ escrito na base $\beta$. Como $\ell$ é uma curva de Lagrangeanos teremos $\mathcal{B}^{T} \omega \mathcal{B}=0$. Derivando duas vezes esta expressão teremos

$$
\begin{gathered}
\dot{\mathcal{B}}^{T} \omega \mathcal{B}+\mathcal{B}^{T} \omega \dot{\mathcal{B}}=0 \\
\ddot{\mathcal{B}}^{T} \omega \mathcal{B}+2 \dot{\mathcal{B}}^{T} \omega \dot{\mathcal{B}}+\mathcal{B}^{T} \omega \ddot{\mathcal{B}}=0 .
\end{gathered}
$$

Como $\mathcal{B}$ é normal teremos $\ddot{\mathcal{B}}=-\mathcal{B} S_{\mathcal{B}}$. Usando isso, e que $\mathcal{B}^{T} \omega \mathcal{B}=0$, na segunda expressão acima teremos $\dot{\mathcal{B}}^{T} \omega \dot{\mathcal{B}}=0$.

O Wronskiano do referencial $\mathcal{B}$ é definido por $W(t)=-\mathcal{B}(t)^{T} \omega \dot{\mathcal{B}}(t)$ (veja Definição 19). Calculando a derivada de $W(t)$

$$
\frac{d}{d t} W(t)=-\dot{\mathcal{B}}(t)^{T} \omega \dot{\mathcal{B}}(t)-\mathcal{B}(t)^{T} \omega \ddot{\mathcal{B}}(t)=\mathcal{B}(t)^{T} \omega \mathcal{B}(t) S_{\mathcal{B}}=0
$$

Logo $W(t)=W_{0}$, onde $W_{0}$ é uma matriz fixa (simétrica e invertível pela Proposição 8). Da mesma forma que acima podemos supor que

$$
W_{0}=I_{n, k}=\left(\begin{array}{cc}
-I_{k} & 0 \\
0 & I_{n-k}
\end{array}\right) .
$$

Calculando

$\left(\mathcal{B}(t) \mid \dot{\mathcal{B}}(t) W_{0}\right)^{T} \omega\left(\mathcal{B}(t) \mid \dot{\mathcal{B}}(t) W_{0}\right)=\left(\begin{array}{cc}\mathcal{B}(t)^{T} \omega \mathcal{B}(t) & \mathcal{B}(t)^{T} \omega \dot{\mathcal{B}}(t) W_{0} \\ W_{0}^{T} \dot{\mathcal{B}}(t)^{T} \omega \mathcal{B}(t) & W_{0}^{T} \dot{\mathcal{B}}(t)^{T} \omega \dot{\mathcal{B}}(t) W_{0}\end{array}\right)=\left(\begin{array}{cc}0 & -W_{0}^{2} \\ \left(W_{0}^{T}\right)^{2} & 0\end{array}\right)=\omega$

Portanto $\left(\mathcal{B}(t) \mid \dot{\mathcal{B}}(t) W_{0}\right)$ é simplética. 
Como $\mathcal{B}$ é normal teremos

$$
\frac{d}{d t}\left(\mathcal{B}(t) \mid \dot{\mathcal{B}}(t) W_{0}\right)=\left(\mathcal{B}(t) \mid \dot{\mathcal{B}}(t) W_{0}\right)\left(\begin{array}{cc}
0 & -S_{\mathcal{B}}(t) W_{0} \\
W_{0} & 0
\end{array}\right) .
$$

Pelo fato que $\left(\mathcal{B}(t) \mid \dot{\mathcal{B}}(t) W_{0}\right)$ é simplética, as contas acima mostram que a última matriz à direita acima tem que estar na álgebra de Lie simplética, e isso implica que $S_{\mathcal{B}}(t) W_{0}$ é simétrica. Equivalentemente

$$
W_{0} S_{\mathcal{B}}(t)^{T}=S_{\mathcal{B}}(t) W_{0} \Leftrightarrow S_{\mathcal{B}}(t)^{T} W_{0}=W_{0} S_{\mathcal{B}}(t) .
$$

E então $W_{0}$ é múltiplo do referencial $\mathcal{B}$, e $\ell$ é variacional.

\subsection{Condição de Comutatividade Generalizada}

Além dessas condições teóricas para uma curva admitir um múltiplo, Sarlet-Engels-Bahar ([SEB82]) desenvolveram um método algébrico para decidir se um dado sistema é variacional ou não. Vamos enunciar este método e obteremos como consequência que: apenas com os invariantes da curva espalhante $\ell$ poderemos decidir se tal curva vem de um problema variacional

Usando a notação da seção anterior temos

$$
\Phi^{(0)}(t)=Q(t)^{T}-\left(P(t)^{2}\right)^{T}-\dot{P}(t)^{T}=S_{\mathcal{A}}(t)^{T} .
$$

Definimos então indutivamente as seguintes matrizes

$$
\Phi^{(j)}(t)=\left[P(t)^{T}, \Phi^{(j-1)}\right]+\frac{d}{d t} \Phi^{(j-1)} .
$$

Escolhendo o mesmo $X(t)$ que em (5.6) (isto é, $X(t)$ satisfaz $\dot{X}=P(t) X$ e $X\left(t_{0}\right)=\mathrm{Id}$ ) para tornar o referencial $\mathcal{B}(t)=\mathcal{A}(t) X(t)$ normal, teremos que $S_{\mathcal{B}}(t)^{T}=\left(X^{-1} S_{\mathcal{A}}(t) X\right)^{T}=$ $X^{T} \Phi^{(0)}(t)\left(X^{-1}\right)^{T}$ e também

$$
\frac{d^{j}}{d t^{j}} S_{\mathcal{B}}(t)^{T}=U^{T} \Phi^{(j)}\left(U^{-1}\right)^{T}
$$

Calculando a expressão acima em $t_{0}$ ficamos com

$$
\left.\frac{d^{j}}{d t^{j}} S_{\mathcal{B}}(t)^{T}\right|_{t=t_{0}}=\Phi^{(j)}\left(t_{0}\right)
$$

isto é, as matrizes $\Phi^{(j)}$ determinam os coeficientes da expansão em Taylor de $S_{\mathcal{B}}^{T}$ em $t_{0}$.

E assim temos o teorema de Sarlet-Engels-Bahar

Teorema 20 (Sarlet-Engels-Bahar(1982)). Assumindo que as matrizes $P$ e $Q$ são analíticas perto de $t_{0}$ então o sistema acima virá de um problema variacional se e somente se existir uma matriz múltipla constante $n \times n$ invertivel e simétrica $W_{0}$, tal que

$$
\Phi^{(j)}\left(t_{0}\right) W_{0}=W_{0} \Phi^{(j)}\left(t_{0}\right)^{T}, \quad j=0,1,2, \ldots
$$

Demonstração. Sabemos que o sistema vem de um problema variacional se, e somente se, existir uma matriz múltipla $n \times n$ invertível e simétrica $W_{0}$ tal que

$$
S_{\mathcal{B}}(t)^{T} W_{0}=W_{0} S_{\mathcal{B}}(t) .
$$

Supondo $P$ e $Q$ analíticas perto de $t_{0}$ a matriz $S_{\mathcal{B}}(t)$ vai ser analítica perto de $t_{0}$. A condição acima é verificada se, e somente se,

$$
\left.\frac{d^{j}}{d t^{j}} S_{\mathcal{B}}(t)^{T}\right|_{t=t_{0}} W_{0}=\left.W_{0} \frac{d^{j}}{d t^{j}} S_{\mathcal{B}}(t)\right|_{t=t_{0}}
$$


ou então,

$$
\Phi^{(j)}\left(t_{0}\right) W_{0}=W_{0} \Phi^{(j)}\left(t_{0}\right)^{T}
$$

As condições acima são de fato mais simples de serem verificadas: é simples de se calcular $\Phi^{(j)}\left(t_{0}\right)$ no ponto $t_{0}$ através $P(t)$ e $Q(t)$ do que resolver a equação diferencial das condições de Helmholtz. Com isso testamos as condições algébricas acima e, se em algum instante essa condição falhar, o sistema não virá de um problema variacional.

Os invariantes da curva espalhante na Grassmanniana permitem reconhecer se a equação linear veio de um problema variacional. Ainda mais: apenas com a primeira condição de comutatividade generalizada (caso $j=0$ no Teorema 20), os invariantes permitirão obter informações dos possíveis Lagrangeanos do problema inverso. Ilustraremos essa ideia abaixo.

Seja $S=S_{\mathcal{A}}\left(t_{0}\right)$ a Schwarziana calculada em $t_{0}$ e $W$ a matriz fixa (candidata à) múltipla. Temos que estudar a equação

$$
S^{T} W=W S,
$$

e para isso vamos separar em alguns casos:

$1^{\circ}$ Caso Todos os autovalores de $S$ reais, diferentes de zero e distintos entre si (condição genérica). Como a equação (5.15) é invariante por mudanças do tipo $X_{0}^{-1} S X_{0}$ e $X_{0} W^{T} X_{0}$, sem perda de generalidade, podemos supor $S$ diagonal tal que cada elemento da diagonal é número real diferente de zero e todos estes números distintos um do outro. Neste caso, esta condição força $W$ a ser do seguinte tipo

$$
W=\left(\begin{array}{cccc}
w_{11} & 0 & \cdots & 0 \\
0 & w_{22} & \cdots & 0 \\
\vdots & \vdots & \ddots & 0 \\
0 & 0 & \cdots & w_{n n}
\end{array}\right),
$$

com $w_{i i}$ sendo números reais não-nulos ( $W$ é invertível).

Neste caso não obtemos nenhuma informação à respeito do Lagrangeano. Porém ao calcular as outras condições de comutatividade poderemos obter mais informação.

$2^{\circ}$ Caso Todos os autovalores de $S$ reais diferentes de zero. Novamente, sem perda de generalidade, podemos supor $S$ diagonal tal que cada elemento da diagonal é um número real diferente de zero. Neste caso, a condição (5.15) força $W$ a ser do seguinte tipo

$$
W=\left(\begin{array}{cccc}
W_{1} & 0 & \cdots & 0 \\
0 & W_{2} & \cdots & 0 \\
\vdots & \vdots & \ddots & 0 \\
0 & 0 & \cdots & W_{l}
\end{array}\right)
$$

com $W_{i}$ matrizes quadradas e invertíveis.

Neste caso também não ganhamos nenhuma informação do Lagrangeano.

$3^{\circ}$ Caso Seja $S$ matriz $4 \times 4$ com 4 autovalores não nulos: 2 autovalores reais e 2 autovalores complexos (com parte imaginária não-nula). Sem perda de generalidade, podemos supor que $S$ se escreve como

$$
S=\left(\begin{array}{cccc}
\lambda_{1} & 0 & 0 & 0 \\
0 & \lambda_{2} & 0 & 0 \\
0 & 0 & a & -b \\
0 & 0 & b & a
\end{array}\right),
$$

com $\lambda_{1}, \lambda_{2}, a, b$ números reais, $\lambda_{1} \neq 0, \lambda_{2} \neq 0$, e $b \neq 0$. Vamos escrever a matriz $W$ em blocos 
para facilitar as contas:

$$
W=\left(\begin{array}{llll}
w_{11} & w_{12} & w_{13} & w_{14} \\
w_{12} & w_{22} & w_{23} & w_{24} \\
w_{13} & w_{23} & w_{33} & w_{34} \\
w_{14} & w_{24} & w_{34} & w_{44}
\end{array}\right)=\left(\begin{array}{c|c|c}
\multicolumn{2}{|c|}{B_{1}} & u^{T} \\
& v^{T} \\
\hline u & v & B_{2}
\end{array}\right),
$$

onde

$$
u=\left(\begin{array}{l}
w_{13} \\
w_{14}
\end{array}\right), \quad v=\left(\begin{array}{l}
w_{23} \\
w_{24}
\end{array}\right) \quad B_{1}=\left(\begin{array}{ll}
w_{11} & w_{12} \\
w_{12} & w_{22}
\end{array}\right) \quad \text { e } \quad B_{2}=\left(\begin{array}{ll}
w_{33} & w_{34} \\
w_{34} & w_{44}
\end{array}\right)
$$

Com isso podemos desenvolver mais facilmente a condição (5.15):

$$
\begin{aligned}
& S^{T} W=W S
\end{aligned}
$$

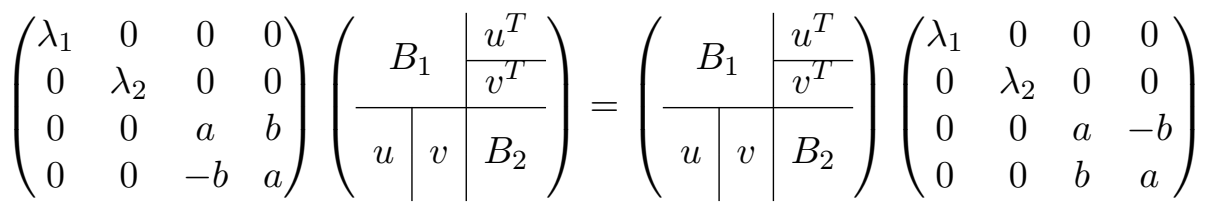

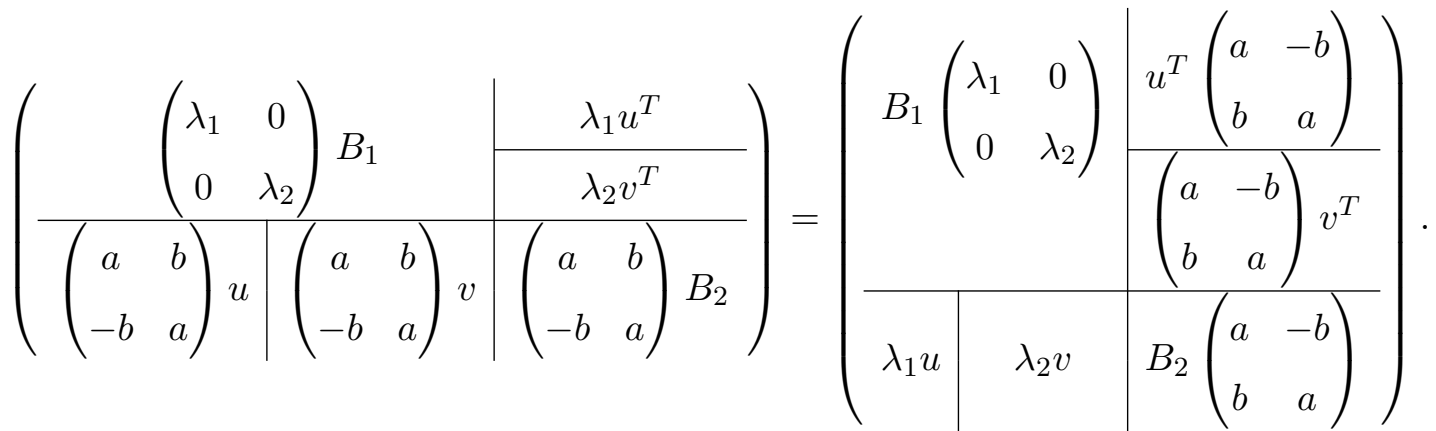

Da igualdade acima temos que ter

$$
\left(\begin{array}{cc}
a & b \\
-b & a
\end{array}\right) u=\lambda_{1} u \quad \text { e } \quad\left(\begin{array}{cc}
a & b \\
-b & a
\end{array}\right) v=\lambda_{2} v
$$

o que implica que $u=v=0$ já que $b \neq 0$. Ou seja, teremos $w_{13}=w_{14}=w_{23}=w_{24}=0$.

Logo a matriz $W$ fica

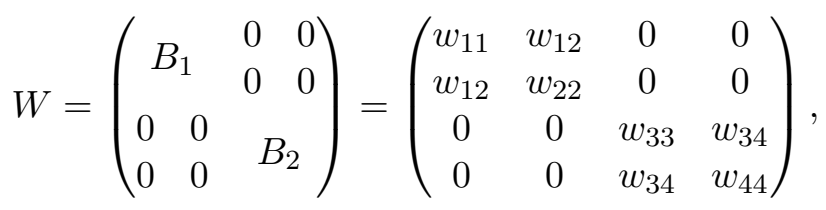

com $B_{1}$ e $B_{2}$ invertíveis.

Ao comparar as matrizes acima teremos que ter também que

$$
\left(\begin{array}{cc}
a & b \\
-b & a
\end{array}\right) B_{2}=B_{2}\left(\begin{array}{cc}
a & -b \\
b & a
\end{array}\right) \Leftrightarrow B_{2}=\left(\begin{array}{cc}
w_{33} & w_{34} \\
w_{34} & -w_{33}
\end{array}\right)
$$

Como $\operatorname{tr}\left(B_{2}\right)=0$ e $B_{2}$ é invertível, a matriz $B_{2}$ possui um autovalor negativo, e o mesmo vale para a matriz $W$. 
Então pelas deduções acima teremos de acima que

$$
W=\left(\begin{array}{ccc}
B_{1} & 0 & 0 \\
0 & 0 & 0 \\
0 & 0 & B_{2}
\end{array}\right)=\left(\begin{array}{cccc}
w_{11} & w_{12} & 0 & 0 \\
w_{12} & w_{22} & 0 & 0 \\
0 & 0 & w_{33} & w_{34} \\
0 & 0 & w_{34} & -w_{33}
\end{array}\right)
$$

e o índice de $W$ será pelo menos 1 .

Neste caso sabemos informação do índice de $W$.

$4^{\circ}$ Caso Suponha que $S$ é matriz $5 \times 5$ e $S$ tem 1 autovalor não-nulo real e 2 autovalores complexos (com parte imaginária não nula) distintos. Sem perda de generalidade podemos supor que $S$ se escreve como

$$
S=\left(\begin{array}{ccccc}
\lambda & 0 & 0 & 0 & 0 \\
0 & a & -b & 0 & 0 \\
0 & b & a & 0 & 0 \\
0 & 0 & 0 & c & -d \\
0 & 0 & 0 & d & c
\end{array}\right)
$$

Da mesma forma que o caso anterior vamos escrever a matriz $W$ em blocos para facilitar as contas:

$$
W=\left(\begin{array}{lllll}
w_{11} & w_{12} & w_{13} & w_{14} & w_{15} \\
w_{12} & w_{22} & w_{23} & w_{24} & w_{25} \\
w_{13} & w_{23} & w_{33} & w_{34} & w_{35} \\
w_{14} & w_{24} & w_{34} & w_{44} & w_{45} \\
w_{15} & w_{25} & w_{35} & w_{45} & w_{55}
\end{array}\right)=\left(\begin{array}{l|l|l|l}
A_{1} & v_{1}^{T} & v_{2}^{T} \\
\hline v_{1} & B_{1} & B_{3}^{T} \\
\hline v_{2} & B_{3} & B_{2}
\end{array}\right)
$$

onde

$$
\begin{gathered}
A_{1}=w_{11}, \quad v_{1}=\left(\begin{array}{l}
w_{12} \\
w_{13}
\end{array}\right), \quad v_{2}=\left(\begin{array}{l}
w_{14} \\
w_{15}
\end{array}\right) \\
B_{1}=\left(\begin{array}{ll}
w_{22} & w_{23} \\
w_{23} & w_{33}
\end{array}\right), \quad B_{2}=\left(\begin{array}{ll}
w_{44} & w_{45} \\
w_{45} & w_{55}
\end{array}\right) \quad \text { e } \quad B_{3}=\left(\begin{array}{ll}
w_{24} & w_{34} \\
w_{25} & w_{35}
\end{array}\right)
\end{gathered}
$$

Com isso, desenvolvendo (5.15) teremos:

$$
S^{T} W=W S
$$

$$
\left(\begin{array}{ccccc}
\lambda & 0 & 0 & 0 & 0 \\
0 & a & b & 0 & 0 \\
0 & -b & a & 0 & 0 \\
0 & 0 & 0 & c & d \\
0 & 0 & 0 & -d & c
\end{array}\right)\left(\begin{array}{c|c|c}
A_{1} & v_{1}^{T} & v_{2}^{T} \\
\hline v_{1} & B_{1} & B_{3}^{T} \\
\hline v_{2} & B_{3} & B_{2}
\end{array}\right)=\left(\begin{array}{c|c|c}
A_{1} & v_{1}^{T} & v_{2}^{T} \\
\hline v_{1} & B_{1} & B_{3}^{T} \\
\hline v_{2} & B_{3} & B_{2}
\end{array}\right)\left(\begin{array}{ccccc}
\lambda & 0 & 0 & 0 & 0 \\
0 & a & -b & 0 & 0 \\
0 & b & a & 0 & 0 \\
0 & 0 & 0 & c & -d \\
0 & 0 & 0 & d & c
\end{array}\right)
$$

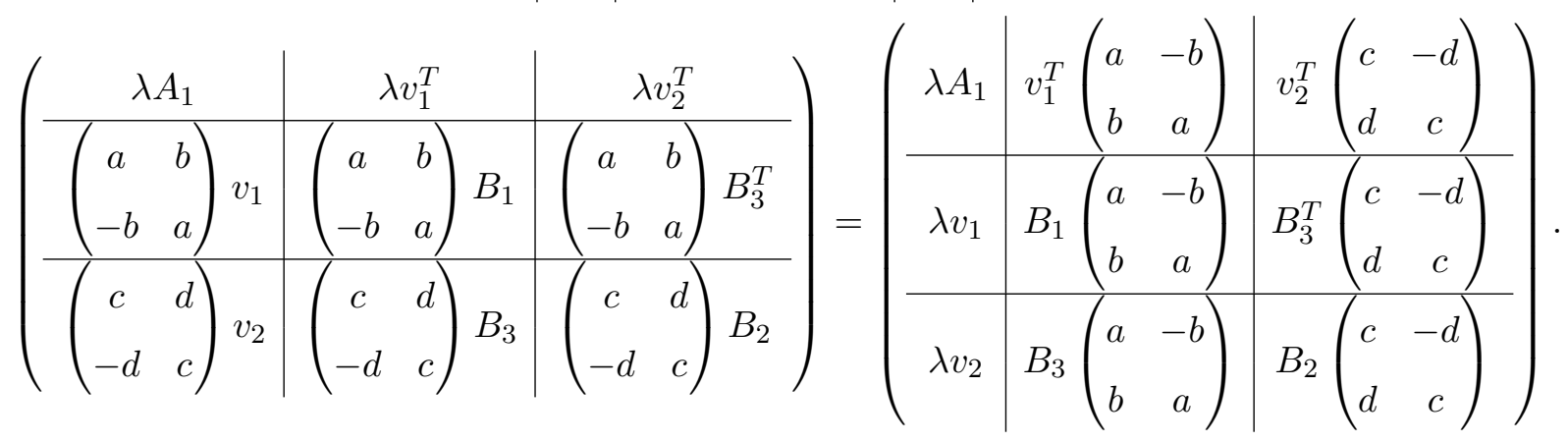


Pela igualdade acima devemos ter

$$
\begin{gathered}
\left(\begin{array}{cc}
a & b \\
-b & a
\end{array}\right) v_{1}=\lambda v_{1}, \quad\left(\begin{array}{cc}
c & d \\
-d & c
\end{array}\right) v_{2}=\lambda v_{2} \\
\left(\begin{array}{cc}
a & b \\
-b & a
\end{array}\right) B_{1}=B_{1}\left(\begin{array}{cc}
a & -b \\
b & a
\end{array}\right), \quad\left(\begin{array}{cc}
c & d \\
-d & c
\end{array}\right) B_{2}=B_{2}\left(\begin{array}{cc}
c & -d \\
d & c
\end{array}\right) \text { e }\left(\begin{array}{cc}
c & d \\
-d & c
\end{array}\right) B_{3}=B_{3}\left(\begin{array}{cc}
a & -b \\
b & a
\end{array}\right) .
\end{gathered}
$$

Como $b \neq 0$ e $d \neq 0$ temos que ter $v_{1}=0$ e $v_{2}=0$, isto é, $w_{12}=w_{13}=w_{14}=w_{15}=0$. Para a segunda linha basta analisar uma equação matricial do seguinte tipo

$$
\left(\begin{array}{cc}
C & D \\
-D & C
\end{array}\right) G=G\left(\begin{array}{cc}
A & -B \\
B & A
\end{array}\right)
$$

onde

$$
G=\left(\begin{array}{cc}
x & y \\
z & w
\end{array}\right)
$$

A equação (5.16) acima é equivalente ao seguinte sistema linear homogêneo

$$
\underbrace{\left(\begin{array}{cccc}
C-A & -B & D & 0 \\
B & C-A & 0 & D \\
-D & 0 & C-A & -B \\
0 & -D & B & C-A
\end{array}\right)}_{H}\left(\begin{array}{l}
x \\
y \\
z \\
w
\end{array}\right)=\left(\begin{array}{l}
0 \\
0 \\
0 \\
0
\end{array}\right) \text {. }
$$

A condição equivalente para que o sistema acima possua mais de uma solução é $\operatorname{det} H=0$, isto é,

$\operatorname{det} H=(C-A)^{4}+2(C-A)^{2} B^{2}+2(C-A)^{2} D^{2}+\left(B^{2}-D^{2}\right)^{2}=0 \Leftrightarrow C=A$ e $D= \pm B$.

E neste caso as soluções serão dadas por

$$
\left(\begin{array}{c}
x \\
y \\
y \\
-x
\end{array}\right) \text { se } C=A \text { e } D=B, \text { ou }\left(\begin{array}{c}
x \\
y \\
-y \\
x
\end{array}\right) \text { se } C=A \text { e } D=-B
$$

isto é, $G$ é dado por

$$
G=\left(\begin{array}{cc}
x & y \\
y & -x
\end{array}\right) \text { se } C=A \text { e } D=B, \text { ou } G=\left(\begin{array}{cc}
x & y \\
-y & x
\end{array}\right) \text { se } C=A \text { e } D=-B
$$

Este desenvolvimento implica que as matrizes $B_{1}, B_{2}$ e $B_{3}$ são dadas por

$$
B_{1}=\left(\begin{array}{cc}
w_{22} & w_{23} \\
w_{23} & -w_{22}
\end{array}\right), \quad B_{2}=\left(\begin{array}{cc}
w_{44} & w_{45} \\
w_{45} & -w_{44}
\end{array}\right) \text { e } \quad B_{3}=\left(\begin{array}{cc}
w_{24} & w_{34} \\
w_{25} & w_{35}
\end{array}\right)=\left(\begin{array}{ll}
0 & 0 \\
0 & 0
\end{array}\right) .
$$

E então a matriz $W$ vai ficar sendo escrita como

$$
\left(\begin{array}{ccccc}
w_{11} & 0 & 0 & 0 & 0 \\
0 & w_{22} & w_{23} & 0 & 0 \\
0 & w_{23} & -w_{22} & 0 & 0 \\
0 & 0 & 0 & w_{44} & w_{45} \\
0 & 0 & 0 & w_{45} & -w_{44}
\end{array}\right)
$$

Note que neste caso o índice de $W$ será pelo menos 2. 
$5^{\circ}$ Caso Suponha que $S$ é matriz $5 \times 5$ e $S$ tem 1 autovalor não-nulo real e 2 autovalores complexos iguais (com parte imaginária não nula). Sem perda de generalidade, podemos supor que $S$ se escreve como

$$
S=\left(\begin{array}{ccccc}
\lambda & 0 & 0 & 0 & 0 \\
0 & a & -b & 0 & 0 \\
0 & b & a & 0 & 0 \\
0 & 0 & 0 & a & -b \\
0 & 0 & 0 & b & a
\end{array}\right)
$$

Da mesma forma que o caso anterior vamos escrever $W$ como :

$$
W=\left(\begin{array}{lllll}
w_{11} & w_{12} & w_{13} & w_{14} & w_{15} \\
w_{12} & w_{22} & w_{23} & w_{24} & w_{25} \\
w_{13} & w_{23} & w_{33} & w_{34} & w_{35} \\
w_{14} & w_{24} & w_{34} & w_{44} & w_{45} \\
w_{15} & w_{25} & w_{35} & w_{45} & w_{55}
\end{array}\right)=\left(\begin{array}{c|c|c|c}
A_{1} & v_{1}^{T} & v_{2}^{T} \\
\hline v_{1} & B_{1} & B_{3}^{T} \\
\hline v_{2} & B_{3} & B_{2}
\end{array}\right),
$$

onde a divisão em blocos é a mesma do caso anterior.

Da mesma forma, desenvolvendo (5.15) teremos:

$$
S^{T} W=W S
$$

$$
\begin{aligned}
& \left(\begin{array}{ccccc}
\lambda & 0 & 0 & 0 & 0 \\
0 & a & -b & 0 & 0 \\
0 & b & a & 0 & 0 \\
0 & 0 & 0 & a & -b \\
0 & 0 & 0 & b & a
\end{array}\right)\left(\begin{array}{c|c|c}
A_{1} & v_{1}^{T} & v_{2}^{T} \\
\hline v_{1} & B_{1} & B_{3}^{T} \\
\hline & & \\
v_{2} & B_{3} & B_{2}
\end{array}\right)=\left(\begin{array}{c|c|c}
A_{1} & v_{1}^{T} & v_{2}^{T} \\
\hline v_{1} & B_{1} & B_{3}^{T} \\
\hline v_{2} & B_{3} & B_{2}
\end{array}\right)\left(\begin{array}{ccccc}
\lambda & 0 & 0 & 0 & 0 \\
0 & a & -b & 0 & 0 \\
0 & b & a & 0 & 0 \\
0 & 0 & 0 & a & -b \\
0 & 0 & 0 & b & a
\end{array}\right)
\end{aligned}
$$

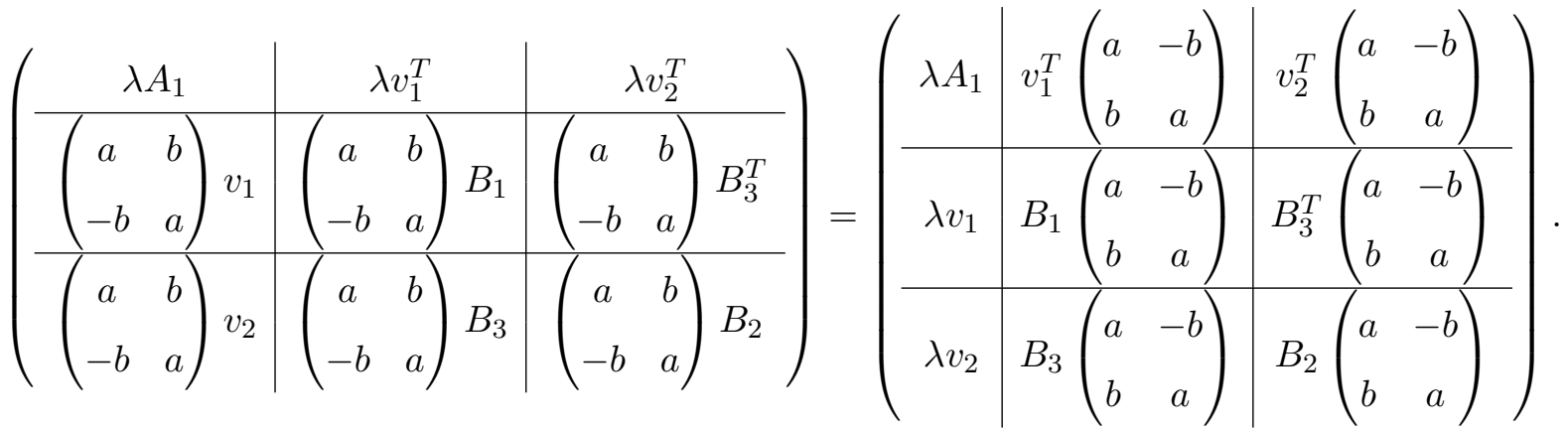

Como no caso anterior temos

$$
\begin{gathered}
\left(\begin{array}{cc}
a & b \\
-b & a
\end{array}\right) v_{1}=\lambda v_{1}, \quad\left(\begin{array}{cc}
a & b \\
-b & a
\end{array}\right) v_{2}=\lambda v_{2}, \\
\left(\begin{array}{cc}
a & b \\
-b & a
\end{array}\right) B_{1}=B_{1}\left(\begin{array}{cc}
a & -b \\
b & a
\end{array}\right), \quad\left(\begin{array}{cc}
a & b \\
-b & a
\end{array}\right) B_{2}=B_{2}\left(\begin{array}{cc}
a & -b \\
b & a
\end{array}\right) \text { e } \quad\left(\begin{array}{cc}
a & b \\
-b & a
\end{array}\right) B_{3}=B_{3}\left(\begin{array}{cc}
a & -b \\
b & a
\end{array}\right),
\end{gathered}
$$

e então

$$
\begin{gathered}
v_{1}=\left(\begin{array}{l}
w_{12} \\
w_{13}
\end{array}\right)=\left(\begin{array}{l}
0 \\
0
\end{array}\right), \quad v_{2}=\left(\begin{array}{l}
w_{14} \\
w_{15}
\end{array}\right)=\left(\begin{array}{l}
0 \\
0
\end{array}\right) \\
B_{1}=\left(\begin{array}{cc}
w_{22} & w_{23} \\
w_{23} & -w_{22}
\end{array}\right), \quad B_{2}=\left(\begin{array}{cc}
w_{44} & w_{45} \\
w_{45} & -w_{44}
\end{array}\right) \text { e } \quad B_{3}=\left(\begin{array}{cc}
w_{24} & w_{25} \\
w_{25} & -w_{24}
\end{array}\right) .
\end{gathered}
$$


Logo $W$ será escrita da seguinte forma

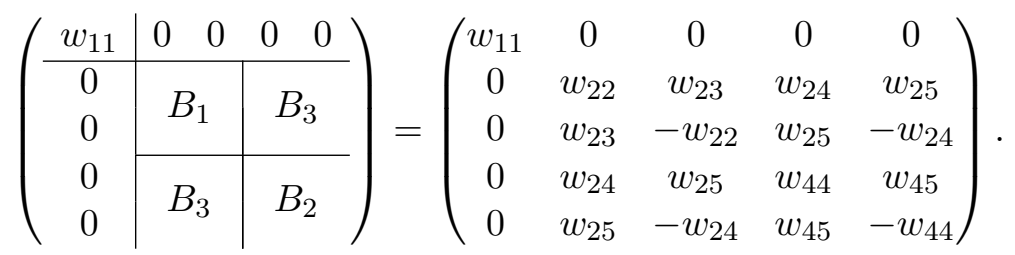

Note que, como a matriz

$$
Z=\left(\begin{array}{cccc}
w_{22} & w_{23} & w_{24} & w_{25} \\
w_{23} & -w_{22} & w_{25} & -w_{24} \\
w_{24} & w_{25} & w_{44} & w_{45} \\
w_{25} & -w_{24} & w_{45} & -w_{44}
\end{array}\right)
$$

é não degenerada e $\operatorname{tr}(Z)=0$, a matriz $Z$ admite pelo menos 1 autovalor negativo, e o mesmo vale para a matriz $W$.

$6^{\circ}$ Caso Caso geral e genérico: suponha $S$ matriz $n \times n$ tal que todos os autovalores de $S$ são não-nulos. Contas análogas aos casos acima podem ser feitas para concluir que $W$ pode ser escrita da seguinte forma

$$
W=\left(\begin{array}{cccccc}
W_{1} & & & & & \\
& \ddots & & & & \\
& & W_{s} & & & \\
& & & Z_{1} & & \\
& & & & \ddots & \\
& & & & & Z_{r}
\end{array}\right),
$$

onde $s$ é o número de autovalores reais distintos, $r$ é o número de autovalores complexos (com parte imaginária diferente de zero) distintos e $W_{i}$ e $Z_{j}$ são matrizes não degeneradas com $\operatorname{tr}\left(Z_{j}\right)=0$ para todo $j$. Note que nesse caso o índice de $W$ será no mínimo o número de auto valores complexos distintos de $S$.

Com essa teoria desenvolvida por Sarlet-Engels-Bahar temos tiramos algumas conclusões a respeito do problema inverso que resumem este último capítulo:

Princípio 1. Sendo $\ell$ uma curva espalhante na Grassmanniana, através dos invariantes em [ÁPD09] é possível

- dizer se tal curva é de Jacobi, isto é, se vem de um problema variacional,

- descobrir o índice mínimo de um possível Lagrangeano. Por exemplo, é possível responder de forma negativa se existe uma métrica Riemanniana ou Lorentz tal que a curva dada na Grassmanniana é a curva de Jacobi de uma tal métrica com índice pequeno, e

- genericamente, com informações da comutatividade generalizada no ponto, é possível resolver o problema inverso. 


\section{Apêndice A}

\section{Problemas Variacionais de Ordem Superior}

Seja $Q$ uma variedade de dimensão $n$ e um intervalo compacto $[a, b]$ que serão fixos ao longo do texto. Um Lagrangeano de ordem $k$ é uma função suave $L: T^{k} Q \rightarrow \mathbb{R}$. O problema variacional de ordem $k$ para o Lagrangeano $L$ no intervalo $[a, b]$ consiste em encontrar curvas $\gamma:[a, b] \rightarrow Q$ que sejam pontos críticos do seguinte funcional

$$
c \mapsto \mathcal{F}(c)=\int_{a}^{b} L\left(j^{k} c(t)\right) d t
$$

onde $c:[a, b] \rightarrow Q$ são curvas na variedade $Q$ e $j^{k} c(t):[a, b] \rightarrow J^{k} Q$ é a prolongação ao $k$ jato da curva $c$ satisfazendo $j^{(k-1)} c(a)=j^{(k-1)} q_{a}$, e $j^{(k-1)} c(b)=j^{(k-1)} q_{b}$ para toda curva $c$, com $j^{(k-1)} q_{a}, j^{(k-1)} q_{b} \in J^{(k-1)} Q$, pontos pré-fixados.

Assumindo uma certa regularidade do Lagrangeano $L$, o objetivo deste capítulo será desenvolver a geometria de tais problemas variacionais. Para mais detalhes consulte [GF00] e [dLR92].

\section{A.1 Problemas Variacionais de Primeira Ordem}

No caso clássico, isto é, quando tivermos um Lagrangeano regular do tipo $L: T Q \rightarrow \mathbb{R}$ a geometria de tais problemas será a geometria simplética dada pela transformação de Legendre ([Cra83])

O problema consiste em encontrar curvas $\gamma$ na variedade $Q$ que são pontos críticos do funcional

$$
c \mapsto \mathcal{F}(c)=\int_{a}^{b} L\left(j^{1} c(t)\right) d t
$$

onde $c:[a ; b] \rightarrow Q$ é uma curva na variedade $Q$ e $j^{1} c(t):[a ; b] \rightarrow T^{1} Q$ é a prolongação ao 1-jato da curva $c$. Neste caso a prolongação será simplesmente a derivada da curva $c$ com relação à $t$, isto é, $j^{1} c(t)=\dot{c}(t)$.

Uma curva $\gamma:[a, b] \rightarrow Q$ será um ponto crítico $\mathcal{F}$ se, e somente se, a primeira variação $\delta^{1} \mathcal{F}$ for identicamente nula no ponto $\gamma$

$$
\delta^{1} \mathcal{F}(\gamma) \equiv 0
$$

A aplicação $\delta^{1} \mathcal{F}(\gamma)$ será um funcional linear definido nos campos ao longo de $\gamma$.

Os campos ao longo de $\gamma$ podem ser identificados com o que chamaremos de variações da curva $\gamma$. Uma variação $\Gamma$ da curva $\gamma$ é uma aplicação do tipo $\Gamma:(-\epsilon, \epsilon) \times \rightarrow Q$ que satisfaz

$$
\Gamma(0, t)=\gamma(t), \quad \Gamma(s, a)=0, \quad \Gamma(s, b)=0,
$$

para todo $s \in(-\epsilon, \epsilon)$. A condição nos extremos $a$ e $b$ é para garantir que a família de curvas 
$\Gamma_{s}=\Gamma(s, \cdot)$ quando variamos o parâmetro $s$ satisfaz a condição das curvas consideradas no problema variacional.

A condição de $\gamma$ ser ponto crítico poderá então ser expressa como

$$
\delta^{1} \mathcal{F}(\gamma)\left(\left.\frac{d}{d s} \dot{\Gamma}_{s}\right|_{s=0}\right)=\left.\frac{d}{d s}\left(\mathcal{F}\left(\dot{\Gamma}_{s}\right)\right)\right|_{s=0}=0
$$

onde $\dot{\Gamma}_{s}$ representa a derivada com relação ao parâmento $t, \dot{\Gamma}_{s}=\frac{d}{d t} \Gamma(s, t)$

Escrevendo em coordenadas locais naturais a variação $\Gamma$ e sua derivada teremos

$$
\Gamma(s, t)=\left(\Gamma_{(0)}(s, t)\right), \quad \dot{\Gamma}(s, t)=\left(\Gamma_{(0)}(s, t), \frac{d}{d t} \Gamma_{(0)}(s, t)\right) .
$$

Denotando por $\left.\frac{d}{d s}\left(\Gamma_{(0)}(s, t)\right)\right|_{s=0}=(h(t))$ temos

$$
\left.\frac{d}{d s} \dot{\Gamma}(s, t)\right|_{s=0}=\left.\frac{d}{d s}\left(\Gamma_{(0)}(s, t), \frac{d}{d t} \Gamma_{(0)}(s, t)\right)\right|_{s=0}=\left(h(t), \frac{d}{d t}(h)(t)\right)
$$

e assim a condição (A.1) fica

$0=\left.\frac{d}{d s} \mathcal{F}\left(\frac{d}{d t} \Gamma_{s}\right)\right|_{s=0}=\int_{a}^{b} d F\left(\left.\frac{d}{d s} \frac{d}{d t} \Gamma(s, t)\right|_{s=0}\right) d t=\int_{a}^{b} \frac{\partial F}{\partial q_{(0)}^{A}}(\dot{\gamma}(t)) h^{A}(t)+\frac{\partial F}{\partial q_{(1)}^{A}}(\dot{\gamma}(t)) \frac{d}{d t}\left(h^{A}(t)\right) d t$.

Usando integrações por partes e observando que $\left(h^{A}\right)(a)=\left(h^{A}\right)(b)=0$ pela definição da variação $\Gamma$, a equação acima fica

$$
0=\left.\frac{d}{d s} \mathcal{F}\left(\frac{d}{d t} \Gamma_{s}\right)\right|_{s=0}=\int_{a}^{b}\left(\frac{\partial L}{\partial q_{(0)}^{A}}(\dot{\gamma}(t))-\frac{d}{d t}\left(\frac{\partial L}{\partial q_{(1)}^{A}}(\dot{\gamma}(t))\right)\right) h^{A}(t) d t .
$$

Como a variação $\Gamma(s, t)$ é arbitrária temos que $\gamma$ deverá satisfazer a seguinte relação diferencial, que é chamada de equação de Euler-Lagrange

$$
\frac{\partial L}{\partial q_{(0)}^{A}}(\dot{\gamma}(t))-\frac{d}{d t}\left(\frac{\partial L}{\partial q_{(1)}^{A}}(\dot{\gamma}(t))\right)=0
$$

para $A=1, \ldots, n$.

A equação acima será de fato uma equação diferencial (de ordem 2) se supormos que a matriz Hessiana de $L$ com relação as velocidades for não-degenerada,

$$
\operatorname{det}\left(\frac{\partial^{2} L}{\partial q_{1}^{A} \partial q_{1}^{B}}\right)_{A B} \neq 0
$$

Supondo isso a equação (A.2) pode ser escrita como

$$
\ddot{\gamma}^{A}(t)=\Gamma^{A}(\gamma(t), \dot{\gamma}(t)) \quad \text { para } A=1 \ldots n .
$$

Com isso podemos definir um campo em $T Q$ cuja a projeção da curva integral será $\gamma$. Este campo será chamado de campo de Euler-Lagrange e será denotado por $\xi_{L}$. O campo de Euler-Lagrange é definido localmente por

$$
\xi_{L}\left(q_{(0)}, q_{(1)}\right)=\left(q_{(0)}, q_{(1)}, q_{(1)}, \xi^{A}\left(q_{(0)}, q_{(1)}\right)\right) .
$$

Se $\phi_{t}$ denotar o fluxo campo $\xi_{L}$, seja $v \in T Q$ e considere a curva $\phi_{t}(v)$ em $T Q$. Esta curva 
satisfaz a seguinte equação diferencial

$$
\frac{d}{d t} \phi_{t}(v)=\xi_{L}\left(\phi_{t}(v)\right)
$$

que localmente fica

$$
\left(\dot{q_{(0)}}(t), \dot{q_{(1)}}(t)\right)=\left(q_{(1)}(t), \xi^{A}\left(q_{(0)}(t), q_{(1)}(t)\right)\right),
$$

e assim

$$
\left\{\begin{array}{l}
q_{(0)}^{\dot{A}}(t)=q_{(1)}^{A}(t) \\
q_{(1)}^{A}(t)=\xi^{A}\left(q_{(0)}(t), q_{(1)}(t)\right)
\end{array} .\right.
$$

Note que as equações (A.2) e (A.3) são as mesmas.

Campos como o que definimos acima que dão origem à equações diferenciais na variedade base são chamados de semi-sprays cuja definição é

Definição 20. Um campo de vetores $\xi: T Q \rightarrow T(T Q)$ é dito um semi spray se qualquer curva integral $\sigma$ de $\xi$ satisfizer

$$
\frac{d}{d t}\left(p_{Q} \circ \sigma\right)(t)=\sigma(t)
$$

Assim uma expressão local do campo $\xi: T Q \rightarrow T(T Q)$ será

$$
\xi=q_{(1)}^{A} \frac{\partial}{\partial q_{(0)}^{A}}+\xi^{A} \frac{\partial}{\partial q_{(1)}^{A}},
$$

e para toda curva $\sigma$ que é uma curva integral de $\xi$, a curva projetada $p_{Q} \circ \sigma$ na variedade $Q$ vai satisfazer uma equação diferencial em coordenadas locais

$$
\frac{d^{2}}{d t^{2}}\left(q_{(0)}^{A}\right)=\xi^{A}\left(q_{(0)}, \frac{d}{d t} q_{(0)}\right)
$$

onde $\sigma(t)=\left(q_{(0)}, q_{(1)}\right)$ é uma expressão local para $\sigma$.

Para mostrar que o campo $\xi_{L}$ pode ser dado de maneira intrínseca precisamos desenvolver um pouco a geometria do espaço tangente $T Q$.

Definição 21. Seja $E$ uma variedade de dimensão $2 m$. Se $E$ admitir um endomorfismo $J: T E \rightarrow$ $T E$ tal que

$$
J^{2}=0, \quad \text { e posto } J=m,
$$

então $J$ é chamada de estrutura quase tangente em $E$.

O espaço tangente $T Q$ admite uma estrutura quase tangente canônica. Considere a seguinte aplicação $J: T(T Q) \rightarrow T(T Q)$ dada pela composição para cada $X \in T(T Q)$

$$
J(X)=i_{v} \circ d\left(p_{Q}\right),
$$

onde $v=p_{T Q}(X)$, com $p_{T Q}: T(T Q) \rightarrow T Q$ a projeção canônica em $T Q, i_{v}$ é a inclusão canônica da fibra $T_{x} Q$ em $T_{v}(T Q)$, com $x=p_{Q}(v)$ e $p_{Q}: T Q \rightarrow Q$ a projeção canônica em $Q$.

Em coordenadas locais teremos que $J$ é dado por

$$
J\left(\frac{\partial}{\partial q_{(0)}^{A}}\right)=\frac{\partial}{\partial q_{(1)}^{A}} \quad \text { e } \quad J\left(\frac{\partial}{\partial q_{(1)}^{A}}\right)=0 .
$$

Portanto $J$ é uma estrutura quase tangente em $Q$.

Temos também o que é chamado de campo de Liouville $C: T Q \rightarrow T(T Q)$ do espaço tangente $T Q$. Ele é definido através da inclusão canônica

$$
C(v)=i_{v}(v)
$$


e em coordenadas locais naturais

$$
C=q_{(1)}^{A} \frac{\partial}{\partial q_{(1)}^{A}} .
$$

Neste caso ainda é importante mencionar o único fibrado vertical $\mathcal{V}(Q)$ que teremos, dado por $\mathcal{V}(Q)=\operatorname{ker} d\left(p_{Q}\right)$. Desta forma teremos que, em coordenadas locais naturais, $\frac{\partial}{\partial q_{(1)}^{A}}$ gera $\mathcal{V}(Q)$, a inclusão canônica $i_{v}: T_{x} Q \rightarrow \mathcal{V}_{v}(Q)$ é um isomorfismo no vertical $\mathcal{V}_{v}(Q)$, e $C(v) \in \mathcal{V}_{v}(Q)$.

O seguinte lema relaciona estes dois conceitos com o conceito de semi-spray

Lema 8. Um campo $\xi: T Q \rightarrow T(T Q)$ será um semi-spray se, e somente se, for satisfeito

$$
J(\xi)=C .
$$

Seguindo [Cra83], para o Lagrangeano $L$ vamos definir a seguinte 2-forma em $T Q$

$$
\omega_{L}=-d(d L \circ J) .
$$

Teremos que $\omega_{L}$ é fechada (pois $\omega_{L}=-d \theta_{L}$, onde $\theta_{L}=(d L \circ J)$ ) e $\omega_{L}$ vai ser não-degenerada se, e somente se, o Lagrangeano $L$ for regular, isto é, se

$$
\operatorname{det}\left(\frac{\partial^{2} L}{\partial q_{(1)}^{A} \partial q_{(1)}^{B}}\right) \neq 0 .
$$

Isso pode ser verificado pela expressão local de $\omega_{L}$,

$$
\omega_{L}=\frac{\partial^{2} L}{\partial q_{(1)}^{A} \partial q_{(0)}^{B}} d q_{(0)}^{A} \wedge d q_{(0)}^{B}+\frac{\partial^{2} L}{\partial q_{(1)}^{A} \partial q_{(1)}^{B}} d q_{(0)}^{A} \wedge d q_{(1)}^{B} .
$$

Vamos definir também a função de energia associada à $L$ dada por

$$
E_{L}=d_{L}(C)-L
$$

que localmente pode ser expressa por

$$
E_{L}=\frac{\partial L}{\partial q_{(1)}^{A}} q_{(1)}^{A}-L
$$

Com estes dois elementos podemos definir o campo $\xi_{L}$ no caso que $L$ é regular

Teorema 21. Suponha o Lagrangeano L regular. Então a única solução que satifaz a equação abaixo para $\xi$

$$
i_{\xi} \omega=E_{L}
$$

vai satisfazer

- $\xi$ é um semi-spray, isto é, $J(\xi)=C$

- as curvas integrais de $\xi$ vão satisfazer Euler-Lagrange, isto é, $\xi=\xi_{L}$.

Antes de passarmos para o caso geral, vamos introduzir o formalismo Hamiltoniano da mecânica clássica. Para isso considere a seguinte função $\operatorname{Leg}_{L}: T Q \rightarrow T^{*} Q$ dada pela seguinte relação

$$
\theta_{L}(X)=\operatorname{Leg}_{L}(v)\left(d\left(p_{Q}\right) X\right)
$$

onde $v=p_{T Q}(X)$. Note que $\operatorname{Leg}_{L}$ está bem definida pela expressão local de $\theta_{L}$.

A função $\operatorname{Leg}_{L}$ acima é chamada de transformação de Legendre da função $L$, e vai satisfazer as seguintes propriedades: 
- $\tau_{Q} \circ \operatorname{Leg}_{L}=p_{Q}$, onde $\tau_{Q}: T^{*} Q \rightarrow Q$ é a projeção canônica,

- $\left(\operatorname{Leg}_{L}\right)^{*} \theta_{0}=\theta_{L}$ e $\left(\operatorname{Leg}_{L}\right)^{*} \omega_{L}=\omega_{0}$, onde $\theta_{0} \in \Omega^{1}\left(T^{*} Q\right)$ e $\omega_{0} \in \Omega^{2}\left(T^{*} Q\right)$ são as 1-forma e 2 -forma canônicas de $T^{*} Q$, respectivamente, e

- a expressão local para a transformação de Legendre é dada por

$$
\operatorname{Leg}_{L}\left(q_{(0)}^{A}, q_{(1)}^{A}\right)=\left(q_{(0)}^{A}, p_{A}^{(0)}\right)
$$

onde

$$
p_{A}^{(0)}=\frac{\partial L}{\partial q_{(1)}^{A}} .
$$

Com isso o seguinte resultado vale

Teorema 22. As seguintes afirmações são equivalentes

- L é regular

- $\omega_{L}$ é simplética

- $\operatorname{Leg}_{L}$ é um difeomorfismo local.

\section{A.2 Geometria dos Espaços Tangentes de Ordem Superior}

Para enunciar o problema do cálculo das variações de ordem superior precisamos da generalização do conceito de velocidade de curvas em uma variedade. Isso é feito utilizando a ideia de jatos.

Definição 22. Considere uma variedade diferenciável $Q$ de dimensão $n$ e um ponto $q \in Q$. Diremos que duas curvas $c_{1}, c_{2}:(-\epsilon, \epsilon) \rightarrow Q$ são $k$-relacionadas em $q$ se $c_{1}(0)=c_{2}(0)=q$ e se para toda função $f: Q \rightarrow \mathbb{R}$ tivermos que todas as $s$ derivadas da função real $f \circ c_{1}-f \circ c_{2}$ em $t=0$ são iguais à 0 , para $s=0,1, \ldots k$.

Esta relação define uma relação de equivalência nas curvas que passam por $q$. Para uma curva $c:(-\epsilon, \epsilon) \rightarrow Q \operatorname{com} c(0)=q$, a classe de equivalência das curvas $k$-relacionadas em $q$ com $c$ será denotada por $j^{k} c(0)$. Chamaremos tal classe de $k$-jato de $c$ no ponto $q$.

A coleção de todos os $k$-jatos em $q$ será denotada por $J_{q}^{k} Q$. E enfim chamamos de espaço tangente de ordem $k$ a seguinte união $T^{k} Q=\cup_{q \in Q} J_{q}^{k} Q$.

É possível mostrar que todos os espaços tangentes de ordem superior admitem uma estrutura diferencial de forma que as seguintes projeções são todas suaves

$$
\rho_{r}^{k}: T^{k} Q \rightarrow T^{r} Q, \quad \rho_{r}^{k}\left(j^{k} c(0)\right)=j^{r} c(0), \quad s=0,1, \ldots k .
$$

Lema 9. O espaço tangente de ordem superior $T^{k} Q$ admite uma estrutura $C^{\infty}$ de variedade diferenciável de modo que para cada ponto $j^{k} c(0)$ de $T^{k} Q$, se denotarmos por $(U, \phi)$ uma carta local no ponto c(0) em $Q$, teremos cartas locais $(\tilde{U}, \tilde{\phi})$ em $T^{k} Q$, onde $\tilde{U}=\left(\rho_{0}^{k}\right)^{-1}(U)$ e

$$
\tilde{\phi}\left(j^{k} \tilde{c}(0)\right)=\left(q_{(0)}, q_{(1)}, \ldots, q_{(k)}\right)=\left(q_{(0)}^{A}, q_{(1)}^{A}, \ldots, q_{(k)}^{A}\right),
$$

$\operatorname{para} j^{k} \tilde{c}(0) \in \tilde{U}, \operatorname{com}_{(s)}=\left(q_{(s)}^{A}\right), A=1, \ldots n, e q_{(s)}=\left.\frac{d^{s}}{d t^{s}}(\phi \circ \tilde{c}(t))\right|_{t=0}$.

Note que $\operatorname{dim} T^{k} Q=(k+1) n$ e teremos ainda que as projeções $\rho_{r}^{k}$ serão todas diferenciáveis com a estrutura descrita acima.

Com a notação do lema anterior as projeções $\rho_{r}^{k}$ nas coordenadas descritas ficam

$$
\rho_{r}^{k}\left(q_{(0)}, \ldots, q_{(k)}\right)=\left(q_{(0)}, \ldots, q_{(r)}\right),
$$


e suas diferenciais

$$
d \rho_{r}^{k}\left(q_{(0)}, \ldots, q_{(k)}, X^{(0)}, \ldots, X^{(k)}\right)=\left(q_{(0)}, \ldots, q_{(r)}, X^{(0)}, \ldots, X^{(r)}\right),
$$

onde $\left(q_{(0)}, \ldots, q_{(r)}, X^{(0)}, \ldots, X^{(r)}\right)$ representa um elemento de $T\left(T^{r} Q\right)$ em coordenadas locais, $r=$ $0,1, \ldots k$.

Os fibrados verticais de ordem superior serão dados por

$$
\mathcal{V}_{r}^{k}(Q)=\operatorname{ker} d \rho_{r}^{k} \subset T\left(T^{k} Q\right),
$$

e localmente podem ser expressos por

$$
\left(q_{(0)}, \ldots, q_{(k)}, 0, \ldots, 0, X^{(r+1)}, \ldots, X^{(k)}\right) .
$$

Além disso podemos levantar diversos objetos de $Q$ ao espaço $T^{k} Q$. Dentre eles citaremos o levantamento de curvas e funções. Considere então uma curva $c$ em $Q$. Existe um levantamento natural para uma curva $j^{k} c$ em $T^{k} Q$ que descreveremos: a curva $j^{k} c(t)$ é definida por

$$
j^{k} c(t)=j^{k} c_{t}(0)
$$

onde $c_{t}$ é uma curva em $Q$ para cada $t$ dada por $c_{t}(s)=c(t+s)$. Se $c(t)=(q(t))$ em coordenadas locais de $Q$, então $j^{k} c(t)$ fica escrito em coordenadas locais de $T^{k} Q$ como

$$
j^{k} c(t)=\left(q(t), \frac{d}{d t} q(t), \ldots, \frac{d^{k}}{d t^{k}} q(t)\right) .
$$

Seja agora uma função real $f: Q \rightarrow \mathbb{R}$. Definimos o $r$-levantamento de $f$ para $T^{k} Q$ como sendo uma função real $f^{(r)}: T^{k} Q \rightarrow \mathbb{R}$ dada por

$$
f^{(r)}\left(j^{k} c(0)\right)=\frac{d^{r}}{d t^{r}}(f \circ c)(0), \quad r=0, \ldots, k .
$$

Note que $f^{(r)}$ está bem definida pela definição de $k$-jatos.

Teremos também as seguintes inclusões canônicas $j_{r}: T^{k} Q \rightarrow T\left(T^{r-1} Q\right), r=1, \ldots k$ dadas por

$$
j_{r}\left(j^{k} c(0)\right)=\left.\frac{d}{d t} j^{r-1} c_{t}(0)\right|_{t=0},
$$

onde $c_{t}(s)=c(t+s)$. Localmente

$$
j_{r}\left(q_{(0)}, \ldots, q_{(k)}\right)=\left(q_{(0)}, \ldots, q_{(r-1)}, q_{(1)}, \ldots, q_{(r)}\right) .
$$

\section{A.2.1 Problema Variacional de Ordem Superior}

Analogamente ao caso de ordem 1, considere agora um Lagrangeano $L: T^{k} Q \rightarrow \mathbb{R}$ de ordem $k$. O problema do cálculo das variações para este Lagrangeano consiste em encontrar pontos críticos do seguinte funcional

$$
c \mapsto \mathcal{F}(c)=\int_{a}^{b} L\left(j^{k} c(t)\right) d t
$$

onde todas as curvas $c:[a, b] \rightarrow Q$ satisfazem $j^{k-1} c(a)=j^{k-1} q_{a}$, e $j^{k-1} c(b)=j^{k-1} q_{b}$, onde $j^{k-1} q_{a}, j^{k-1} q_{b} \in T^{k-1} Q$ são pontos pré-fixados.

Sendo $c:[a, b] \rightarrow Q$ uma curva em $Q$ considere uma variação $\Gamma:(-\epsilon, \epsilon) \times[a, b] \rightarrow Q$ de $c$, isto é

$$
\Gamma(0, t)=c(t), \quad j^{k-1} \Gamma(s, a)=j^{k-1} q_{a}, \quad j^{k-1} \Gamma(s, b)=j^{k-1} q_{b}
$$

onde a prolongação em $\Gamma=\Gamma(s, t)$ refere-se à prolongação ao tangente de ordem $k-1$ com relação 
à variável $t$.

Uma curva $\gamma:[a, b] \rightarrow Q$ será um ponto crítico $\mathcal{F}$ se, e somente se, a primeira variação $\delta^{1} \mathcal{F}$ for identicamente nula no ponto $\gamma$

$$
\delta^{1} \mathcal{F}(\gamma) \equiv 0
$$

A aplicação $\delta^{1} \mathcal{F}(\gamma)$ será um funcional linear definido nos campos ao longo de $\gamma$, que podemos identificar com a derivada das variações $\Gamma(s, t)$ com relação ao parâmetro $s$ em 0 . Assim, uma curva $\gamma$ será ponto crítico de $\mathcal{F}$ se, e somente se, para toda variação $\Gamma$ de $\gamma$ tivermos

$$
\delta^{1} \mathcal{F}(\gamma)\left(\left.\frac{d}{d s} j^{k} \Gamma_{s}\right|_{s=0}\right)=\left.\frac{d}{d s}\left(\mathcal{F}\left(j^{k} \Gamma_{s}\right)\right)\right|_{s=0}=0
$$

onde $j^{k} \Gamma_{s}$ é a uma família de curvas no parâmetro $s$ construída pela prolongação ao espaço tangente de ordem $k$ com relação à varável $t$ de $\Gamma(s, t)$.

Escrevendo em coordenadas locais naturais a variação $\Gamma$ e sua prolongação teremos

$$
\Gamma(s, t)=\left(\Gamma_{(0)}(s, t)\right), \quad j^{k} \Gamma(s, t)=\left(\Gamma_{(0)}(s, t), \frac{d}{d t} \Gamma_{(0)}(s, t), \ldots, \frac{d^{k}}{d t^{k}} \Gamma_{(0)}(s, t)\right) .
$$

Denotando por $\left.\frac{d}{d s}\left(\Gamma_{(0)}(s, t)\right)\right|_{s=0}=(h(t))$ temos

$$
\left.\frac{d}{d s} j^{k} \Gamma(s, t)\right|_{s=0}=\left.\frac{d}{d s}\left(\Gamma_{(0)}(s, t), \frac{d}{d t} \Gamma_{(0)}(s, t), \ldots, \frac{d^{k}}{d t^{k}} \Gamma_{(0)}(s, t)\right)\right|_{s=0}=\left(h(t), \frac{d}{d t}(h)(t), \ldots, \frac{d^{k}}{d t^{k}}(h)(t)\right)
$$

e assim a condição (A.4) fica

$$
0=\left.\frac{d}{d s} \mathcal{F}\left(j^{k} \Gamma_{s}\right)\right|_{s=0}=\int_{a}^{b} d F\left(\left.\frac{d}{d s} j^{k} \Gamma(s, t)\right|_{s=0}\right) d t=\int_{a}^{b} \sum_{i=0}^{k} \frac{\partial F}{\partial q_{(i)}^{A}}\left(j^{k} \gamma(t)\right) \frac{d^{i}}{d t^{i}}\left(h^{A}\right)(t) d t .
$$

Usando diversas integrações por partes e observando que $\frac{d^{i}}{d t^{i}}\left(h^{A}\right)(a)=\frac{d^{i}}{d t^{i}}\left(h^{A}\right)(b)=0, i=$ $0,1, \ldots, k-1$, a equação acima fica

$$
0=\left.\frac{d}{d s} \mathcal{F}\left(j^{k} \Gamma_{s}\right)\right|_{s=0}=\int_{a}^{b}\left(\sum_{i=0}^{k}(-1)^{i} \frac{d^{i}}{d t^{i}}\left(\frac{\partial L}{\partial q_{(i)}^{A}}\left(j^{k} \gamma(t)\right)\right)\right) h^{A}(t) d t .
$$

Como a variação $\Gamma(s, t)$ é arbitrária temos que $\gamma$ deverá satisfazer a seguinte relação diferencial, que é chamada de equação de Euler-Lagrange

$$
\sum_{i=0}^{k}(-1)^{i} \frac{d^{i}}{d t^{i}}\left(\frac{\partial L}{\partial q_{(i)}^{A}}\left(j^{k} \gamma(t)\right)\right)=0
$$

para $A=1, \ldots, n$.

No decorrer das seções desenvolveremos a formulação intrínseca do cálculo das variações.

\section{A.2.2 Equações Diferenciais e Estrutura Quase Tangente}

Antes de prosseguir definindo a generalização da estrutura quase tangente canônica para espaços tangentes de ordem superior, vamos definir o que seria uma equação diferencial de ordem $k$ na variedade $Q$. Uma vez que a solução do problema variacional irá satisfazer uma certa equação diferencial, este conceito será importante para enunciarmos de maneira intrínseca as equações de Euler-Lagrange.

Definição 23. Um campo de vetores $\xi: T^{k} Q \rightarrow T\left(T^{k} Q\right)$ é dito um semi spray se qualquer curva integral $\sigma$ satisfizer

$$
j^{k}\left(\rho_{0}^{k} \circ \sigma\right)(t)=\sigma(t)
$$


Em coordenadas locais, a definição acima significa que cada coordenada de $\sigma$ será a derivada da coordenada anterior, isto é, teremos

$$
\sigma(t)=\left(q_{(0)}(t), q_{(1)}(t), q_{(2)}(t), \ldots, q_{(k)}(t)\right)=\left(q_{(0)}(t), \frac{d}{d t}\left(q_{(0)}(t)\right), \frac{d}{d t}\left(q_{(1)}(t)\right), \ldots, \frac{d}{d t}\left(q_{(k-1)}(t)\right)\right)
$$

Portanto o campo $\xi$ fica escrito localmente por

$$
\xi=\sum_{i=0}^{k-1} q_{(i+1)}^{A} \frac{\partial}{\partial q_{(i)}^{A}}+\xi^{A} \frac{\partial}{\partial q_{(k)}^{A}},
$$

onde $\xi^{A}$ são funções em $T^{k} Q$.

Vamos usar as projeções $\rho_{r-1}^{k}$ e os espaços verticais para construir o que são chamados de endomorfismos verticais em $T\left(T^{k} Q\right)$ e uma estrutura quase tangente generalizada.

Primeiramente note que $\mathcal{V}_{r-1}^{k} Q$ é um fibrado sobre $T^{k} Q$ de modo que um elemento $X \in$ $\mathcal{V}_{r-1}^{k}(Q) \subset T\left(T^{k} Q\right)$ pode ser escrito localmente por

$$
\left(q_{(0)}, \ldots, q_{(k)}, X^{(r)}, \ldots, X^{(k)}\right)
$$

onde a expressão local de $X$ em $T\left(T^{k} Q\right)$ é

$$
\left(q_{(0)}, \ldots, q_{(k)}, 0, \ldots, 0, X^{(r)}, \ldots, X^{(k)}\right) .
$$

Assim a inclusão canônica $i_{k-r+1}: \mathcal{V}_{r-1}^{k} Q \rightarrow T\left(T^{k} Q\right)$ com relação à estas coordenadas locais fica

$$
i_{k-r+1}\left(q_{(0)}, \ldots, q_{(k)}, X^{(r)}, \ldots, X^{(k)}\right)=\left(q_{(0)}, \ldots, q_{(k)}, 0, \ldots, 0, X^{(r)}, \ldots, X^{(k)}\right) .
$$

Seja a seguinte projeção canônica $p_{T^{r-1} Q}: T\left(T^{r-1} Q\right) \rightarrow T^{r-1} Q$ e a projeção $\rho_{r-1}^{k}: T^{k} Q \rightarrow$ $T^{r-1} Q$ definida acima. Considere o fibrado pullback de $T\left(T^{r-1} Q\right)$ por $\rho_{r-1}^{k}$, que iremos denotar por $T^{k} Q \times{ }_{T^{r-1} Q} T\left(T^{r-1} Q\right)$. Este fibrado pullback será dado pelos pares ordenados $\left(j^{k} c(0), X\right) \in$ $T^{k} Q \times T\left(T^{r-1} Q\right)$ tais que $\rho_{r-1}^{k}\left(j^{k} c(0)\right)=p_{T^{r-1} Q}(X)$.

O fibrado $T^{k} Q \times_{T^{r-1} Q} T\left(T^{r-1} Q\right)$ será um fibrado vetorial sobre $T^{k} Q$ de forma que o seguinte diagrama comuta

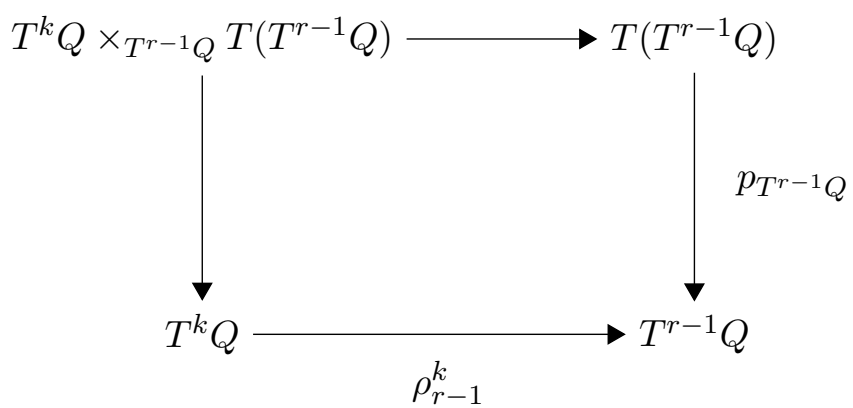

Um elemento $\left(j^{k} c(0), X\right) \in T^{k} Q \times_{T^{r-1} Q} T\left(T^{r-1} Q\right)$ pode ser expresso localmente por

$$
\left(j^{k} c(0), X\right)=\left(q_{(0)}, \ldots, q_{(k)}, q_{(0)}, \ldots, q_{(r-1)}, X^{(0)}, \ldots, X^{(r-1)}\right)
$$

Além disso teremos os homomorfismos de fibrados vetoriais $s_{k-r+1}: T\left(T^{k} Q\right) \rightarrow T^{k} Q \times_{T^{r-1} Q}$ $T\left(T^{r-1}\right) Q$ sobre $T^{k} Q$ dados por $s_{k-r+1}(X)=\left(p_{T^{k} Q}(X), d \rho_{r-1}^{k}(X)\right)$, e localmente por

$$
s_{k-r+1}\left(q_{(0)}, \ldots, q_{(k)}, X^{(0)}, \ldots, X^{(k)}\right)=\left(q_{(0)}, \ldots, q_{(k)}, q_{(0)}, \ldots, q_{(r-1)}, X^{(0)}, \ldots, X^{(r-1)}\right)
$$

Assim o seguinte diagrama também irá comutar 


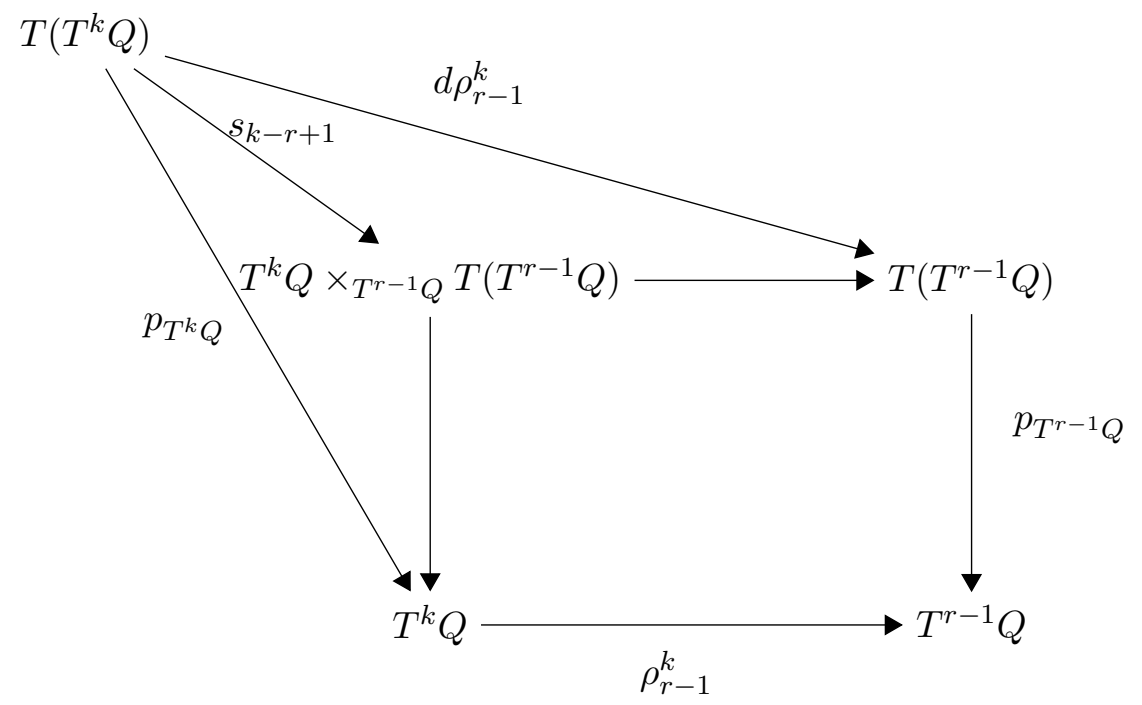

Todas essas considerações foram feitas para enunciar a seguinte definição da estrutura geométrica de $T^{k} Q$ e suas projeções

Definição 24. Temos $k$ sequências de fibrados vetoriais dadas por

$$
0 \rightarrow \mathcal{V}_{r-1}^{k} Q \stackrel{i_{k-r+1}}{\longrightarrow} T\left(T^{k} Q\right) \stackrel{s_{k-r+1}}{\longrightarrow} T^{k} Q \times_{T^{r-1} Q} T\left(T^{r-1} Q\right) \rightarrow 0,
$$

$r=1, \ldots, k$, de modo que cada uma delas será exata.

Chamamos a sequência acima de $(k-r+1)$-éssima sequência exata fundamental

Com um pouco mais de detalhes as $k$ sequências fundamentais são

$$
\begin{array}{ll}
\left(1^{\mathrm{a}}\right) \quad 0 \rightarrow \mathcal{V}_{k-1}^{k} Q \stackrel{i_{1}}{\rightarrow} T\left(T^{k} Q\right) \stackrel{s_{1}}{\rightarrow} T^{k} Q \times_{T^{k-1} Q} T\left(T^{k-1} Q\right) \rightarrow 0 \\
\quad \vdots \\
\left(\mathrm{r}^{\mathrm{a}}\right) \quad 0 \rightarrow \mathcal{V}_{k-r}^{k} Q \stackrel{i_{r}}{\longrightarrow} T\left(T^{k} Q\right) \stackrel{s_{r}}{\longrightarrow} T^{k} Q \times_{T^{k-r} Q} T\left(T^{k-1} Q\right) \rightarrow 0 \\
\quad \vdots \\
\left(\mathrm{k}^{\mathrm{a}} \quad \quad 0 \rightarrow \mathcal{V}_{0}^{k} Q \stackrel{i_{k}}{\rightarrow} T\left(T^{k} Q\right) \stackrel{s_{k}}{\rightarrow} T^{k} Q \times_{Q} T Q \rightarrow 0\right.
\end{array}
$$

Para cada sequência acima temos um isomorfismo de fibrados vetoriais

$$
h_{r}: T^{k} Q \times_{T^{r-1} Q} T\left(T^{r-1} Q\right) \rightarrow \mathcal{V}_{k-r}^{k} Q,
$$

de modo que as sequências fundamentais ficam conectadas pelos diagramas

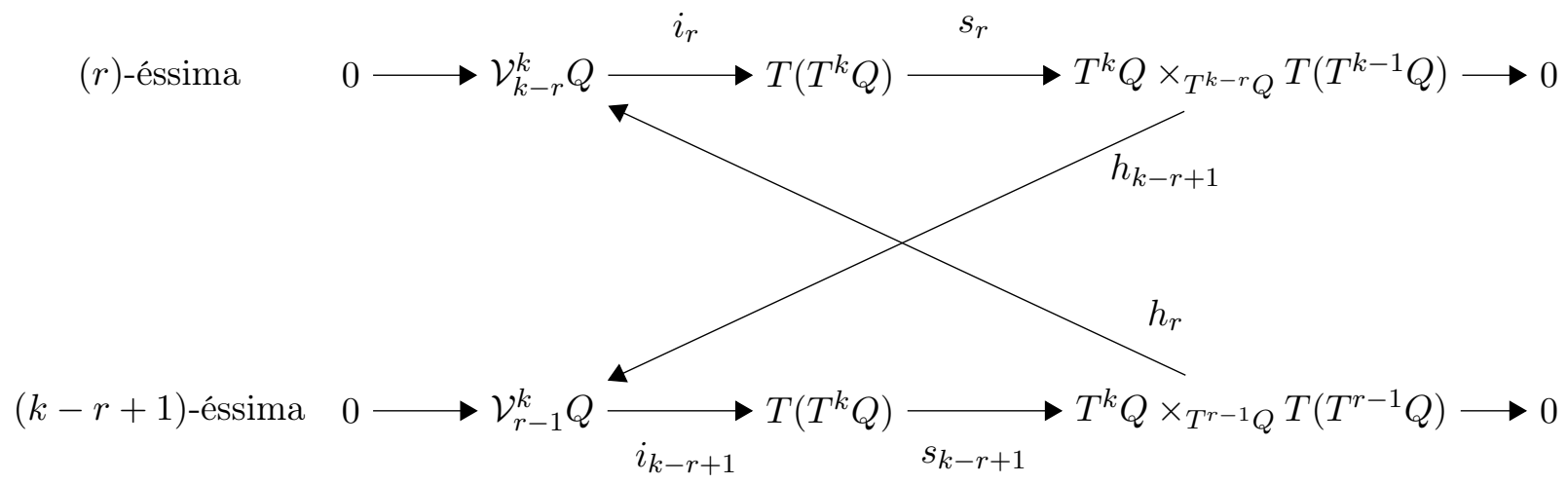


A expressão local de $h_{r}$ é dada por

$h_{r}\left(q_{(0)}, \ldots, q_{(k)}, q_{(0)}, \ldots, q_{(r-1)}, X^{(0)}, \ldots, X^{(r-1)}\right)=\left(q_{(0)}, \ldots, q_{(k)}, 0, \ldots, 0, X^{(0)}, \ldots, X^{(r-1)}\right)$

Através dessas sequências teremos os endomorfismos verticais

Definição 25. O r-éssimo endomorfismo vertical $J_{r}: T\left(T^{k} Q\right) \rightarrow T\left(T^{k} Q\right)$ é dado por

$$
J_{r}=i_{k-r+1} \circ h_{k-r+1} \circ s_{r}, \quad r=1, \ldots, k .
$$

Pelas expressões locais de $i_{k-r+1}, h_{k-r+1}$ e $s_{r}, J_{r}$ será dado por

$$
J_{r}\left(q_{(0)}, \ldots, q_{(k)}, X^{(0)}, \ldots, X^{(k)}\right)=\left(q_{(0)}, \ldots, q_{(k)}, 0, \ldots, 0, X^{(0)}, \ldots, X^{(k-r)}\right),
$$

e teremos a seguinte proposição

Proposição 10. O r-éssimo endomorfismo vertical $J_{r}$ tem posto $r m$ e satisfaz

$$
\left(J_{r}\right)^{s}= \begin{cases}0, & \text { se } r s \geq k+1 \\ J_{r s}, & \text { se } r s<k+1\end{cases}
$$

- $\operatorname{ker} J_{r}=\mathcal{V}_{k-r}^{k} Q=\operatorname{Im} J_{k-r+1}$

- $\operatorname{Im} J_{k} \subset \ldots \subset \operatorname{Im} J_{2} \subset \operatorname{Im} J_{1}$.

Com isso fica claro que $J_{1}$ será uma estrutura quase tangente de ordem superior em $T^{k} Q$.

Definição 26. Seja $E$ uma variedade de dimensão $(k+1) m$. Se $E$ admitir um endomorfismo $J: T E \rightarrow T E$ tal que

$$
J^{k+1}=0 \quad \text { e } \quad \operatorname{dim} \operatorname{Im} J=k m,
$$

então $J$ é dita uma estrutura quase tangente de ordem $k$ em $E$.

Proposição 11. O primeiro endomorfismo vertical $J_{1}$ será uma estrutura quase tangente de ordem k em $T^{k} Q$.

$J_{1}$ é chamado de estrutura quase tangente canônica de ordem $k$ em $T^{k} Q$.

Antes de encerrar esta seção vamos definir os campos de Liouville neste contexto mais geral.

Definição 27. Considere o seguinte campo vetorial $C_{r}$ em $T^{k} Q$ dado pela composição

$$
C_{r}=h_{k-r+1} \circ\left(\mathrm{Id} \times j_{k-r+1}\right),
$$

para $r=1, \ldots k$.

Pela definição, $C_{r}$ é de fato um campo vetorial em $T^{k} Q$, e é chamado de $r$-éssimo campo vetorial canônico em $T^{k} Q$. O campo vetorial $C_{1}$ também é chamado de campo de Lioville de ordem $k$.

Pelas expressões locais de $h_{k-r+1}$ e $j_{k-r+1}$, o $r$-éssimo campo vetorial canônico $C_{r}$ fica escrito localmente como

$$
C_{r}=\sum_{i=1}^{k-r+1} q_{(i)}^{A} \frac{\partial}{\partial q_{(r+i-1)}},
$$

e o campo de Liouville fica

$$
C_{1}=\sum_{i=1}^{k} q_{(i)}^{A} \frac{\partial}{\partial q_{(i)}} .
$$

Através do campo de Liouville e da estrutura quase tangente conseguimos caracterizar os semisprays da mesma forma que em ordem 1 
Lema 10. Um campo $\xi: T^{k} Q \rightarrow T\left(T^{k} Q\right)$ será um semi-spray se, e somente se, for satisfeito

$$
J_{1}(\xi)=C_{1} .
$$

\section{A.2.3 Derivações Verticais}

Nesta seção enunciaremos resultados desenvolvidos por Leon-Rodrigues ([dLR92]) sobre o cálculo exterior nos espaços tangente de ordem superior. Usaremos os conceitos de derivação de álgebra exterior que podem ser encontrados em [KN69].

Definição 28. Seja $\omega$ uma $p$-forma em $T^{k} Q, p \geq 1$. Definimos o produto interno de $J_{r}$ por $\omega$ como sendo a $p$-forma em $T^{k} Q$ dada por

$$
i_{J_{r}} \omega\left(X_{1}, \ldots, X_{p}\right)=\sum_{i=1}^{p} \omega\left(X_{1}, \ldots, J_{r} X_{i}, \ldots, X_{p}\right)
$$

Também definimos $i_{J_{r}} f=0$ para qualquer função real $f: T^{k} Q \rightarrow \mathbb{R}$.

O mapa $\omega \mapsto i_{J_{r}} \omega$ é chamado de $r$-éssima derivação em $\Lambda\left(T^{k} Q\right)$.

A $r$-éssima derivação $i_{J_{r}}$ estará completamente determinada pela ação nas funções e nas 1-formas exatas de $T^{k} Q$

Proposição 12. O mapa $\omega \mapsto i_{J_{r}} \omega$ é a única derivação de grau 0 na álgebra exterior $\Lambda\left(T^{k} Q\right)$ que satisfaz para qualquer função $f$ em $T^{k} Q$ o seguinte

- $i_{J_{r}} f=0$

- $i_{J_{r}}(d f)=J_{r}^{*}(d f)$.

Localmente teremos

$$
i_{J_{r}}\left(d q_{(i)}^{A}\right)= \begin{cases}0, & \text { se } i<r \\ d q_{(i-r)}^{A}, & \text { se } i \geq r\end{cases}
$$

para $i=0,1, \ldots, k$ e $A=1, \ldots, n$.

Com isso podemos definir uma anti-derivação de grau 1 em $\Lambda\left(T^{k} Q\right)$

Definição 29. O seguinte mapa $\omega \mapsto d_{J_{r}} \omega$ na álgebra exterior dado pelo comutador $d_{J_{r}}=\left[i_{J_{r}}, d\right]=$ $i_{J_{r}} d-d i_{J_{r}}$ é chamado de $r$-éssima derivação vertical.

Proposição 13. O mapa $\omega \mapsto d_{J_{r}} \omega$ é a única anti-derivação de grau 1 na álgebra exterior $\Lambda\left(T^{k} Q\right)$ que satisfaz para qualquer função $f$ em $T^{k} Q$ o seguinte

- $d_{J_{r}} f=J_{r}^{*}(d f)$

- $d_{J_{r}}(d f)=-d\left(J_{r}^{*}(d f)\right)$.

Localmente teremos

- $d_{J_{r}} f=\sum_{i=r}^{k} \frac{\partial f}{\partial q_{(i)}^{A}} d q_{(i-r)}^{A}$

- $d_{J_{r}}\left(d q_{(i)}^{A}\right)=0, i=0,1, \ldots, k$. 


\section{A.2.4 Derivação de Tulczyjew}

Para introduzirmos a derivação de Tulczyjew, que irá generalizar o conceito derivada com relação ao parâmetro de tempo de curvas em $T^{k} Q$, primeiramente precisamos encontrar uma forma de relacionar formas diferenciáveis de diferentes espaços tangentes de ordem superior. Assim conseguiremos construir uma álgebra exterior $\Lambda$ que irá ser o conjunto das uniões das álgebras exteriores $\cup_{k=0}^{\infty} \Lambda\left(T^{k} Q\right)$.

Para identificar duas formas $\mu \in \Lambda\left(T^{k} Q\right)$ e $\nu \in \Lambda\left(T^{k^{\prime}} Q\right)$ usamos a seguinte relação de equivalência: $\mu$ é equivalente à $\nu$ se $k^{\prime} \leq k$ e $\mu=\left(\rho_{k^{\prime}}^{k}\right)^{*} \nu$, ou se $k \leq k^{\prime}$ e $\nu=\left(\rho_{k}^{k^{\prime}}\right)^{*} \mu$.

Com a relação descrita acima o operador de diferenciação exterior $d$ e o produto exterior $\wedge$ se estendem de maneira natural à $\Lambda$, e assim, $\Lambda$ se torna uma álgebra graduada comutativa. Essa álgebra exterior foi construída em casos bem mais gerais por Anderson ([And]).

Para definir o operador de Tulczyjew em $\Lambda$ seja primeiro uma função $f: T^{k} Q \rightarrow \mathbb{R}$. Definiremos uma função $d_{T} f: T^{k+1} Q \rightarrow \mathbb{R}$ dada por

$$
\left(d_{T} f\right)\left(j^{k+1} c(0)\right)=d f_{j^{k} c(0)}\left(j_{k}\left(j^{k+1} c(0)\right)\right)
$$

onde $j_{k}: T^{k+1} \rightarrow T\left(T^{k} Q\right)$ é a inclusão canônica descrita acima. Localmente teremos

$$
\left(d_{T} f\right)\left(q_{(0)}, \ldots, q_{(k)}\right)=\sum_{i=0}^{k} \frac{\partial f}{\partial q_{(i)}^{A}} q_{(i+1)}^{A},
$$

e podemos estender este operador à $\Lambda$

Proposição 14. Existe um único operador $\omega \mapsto d_{T} \omega$ em $\Lambda$ que é uma derivação de grau 0 tal que localmente satisfaz (A.6) $e$

$$
d_{T} d q_{(i)}^{A}=q_{(i+1)}^{A}, \quad i=0,1, \ldots, k .
$$

Além disso teremos que $d_{T} d=d d_{T}$, e os operadores $i_{J_{r}}$ e $d_{J_{r}}$ se estendem à $\Lambda$ de maneira natural.

\section{A.2.5 Formalismo de Klein}

Nesta seção apresentamos o formalismo intrínseco do problema do cálculo das variações.

Seja $L: T^{k} Q \rightarrow \mathbb{R}$ um Lagrangeano de ordem $k$. Definimos a seguinte 2-forma fechada em $T^{2 k-1} Q$

$$
\omega_{L}=\sum_{i=1}^{k}(-1)^{i} d_{T}^{i-1} d d_{J_{i}} L .
$$

Note na verdade que $\omega$ é uma forma exata. Basta observar que $\omega=-d \theta_{L}$, com $\theta_{L}$ dado por

$$
\theta_{L}=\sum_{i=1}^{k}(-1)^{i-1} d_{T}^{i-1} d_{J_{i}} L
$$

e a justificativa desta conta é porque os operadores $d$ e $d_{T}$ comutam, $d d_{T}=d_{T} d$.

Seja também a seguinte função $E: T^{2 k-1} Q \rightarrow \mathbb{R}$, chamada de energia de $L$, dada por

$$
E_{L}=\sum_{i=0}^{k-1}(-1)^{i} d_{T}^{i}\left(C_{i+1} L\right)-L .
$$


As expressões locais de $\theta_{L}, \omega_{L}$ e $E_{L}$ são

$$
\begin{gathered}
\theta_{L}=\sum_{r=1}^{k} \sum_{i=0}^{k-r}(-1)^{i} d_{T}^{i}\left(\frac{\partial L}{\partial q_{(r+i)}^{A}}\right) d q_{(r-1)}^{A} \\
\omega_{L}=\sum_{r=1}^{k} \sum_{i=0}^{k-r}(-1)^{i+1} d_{T}^{i} d\left(\frac{\partial L}{\partial q_{(r+i)}^{A}}\right) d q_{(r-1)}^{A} \\
E_{L}=\sum_{r=1}^{k} q_{r}^{A} \sum_{i=0}^{k-r}(-1)^{i} d_{T}^{i}\left(\frac{\partial L}{\partial q_{(r+i)}^{A}}\right)-L .
\end{gathered}
$$

Com estes elementos é possível expressar o que é um ponto crítico do funcional $\mathcal{F}$ de maneira intrínseca

Teorema 23. Seja $L: T^{k} Q \rightarrow \mathbb{R}$ um Lagrangeano de ordem $k$ regular, isto é,

$$
\operatorname{det}\left(\frac{\partial^{2} L}{\partial q_{(k)}^{A} \partial q_{(k)}^{B}}\right) \neq 0
$$

em todo ponto de $T^{k} Q$.

Então existirá apenas uma única solução da equação

$$
i_{\xi} \omega=d\left(E_{L}\right)
$$

na variável $\xi: T^{2 k-1} Q \rightarrow T\left(T^{2 k-1} Q\right)$.

Tal solução, que denotamos por $\xi_{L}$ e chamamos de campo de Euler-Lagrange, satisfaz

- $\xi_{L}$ é um semi-spray, $e$

- as curvas integrais de $\xi_{L}$ satisfazem a equação de Euler-Lagrange (A.5) de L.

\section{A.2.6 Transformação de Legendre}

A transformação de Legendre irá possibilitar a passagem do contexto Lagrangeano para o Hamiltoniano. Analogamente ao caso de ordem 1, definimos a transformação de Legendre $\operatorname{Leg}_{L}$ : $T^{2 k-1} Q \rightarrow T^{*}\left(T^{k-1} Q\right)$ associada ao Lagrangeano $L$ dada pela seguinte relação para cada $X \in$ $T\left(T^{2 k-1} Q\right)$

$$
\theta_{L}(X)=\operatorname{Leg}_{L}\left(p_{T^{2 k-1} Q}(X)\right)\left(d \rho_{k-1}^{2 k-1}(X)\right) .
$$

Assim $\operatorname{Leg}_{L}$ irá satisfazer as seguintes propriedades

- $\tau_{T^{k-1} Q} \circ \operatorname{Leg}_{L}=\rho_{k-1}^{2 k-1}$, onde $\tau_{T^{k-1} Q}: T^{*}\left(T^{k-1} Q\right) \rightarrow T^{k-1} Q$ é a projeção canônica.

- $\left(\operatorname{Leg}_{L}\right)^{*} \theta_{k-1}=\theta_{L}$ e $\left(\operatorname{Leg}_{L}\right)^{*} \omega_{k-1}=\omega_{L}$, onde $\theta_{k-1} \in \Omega^{1}\left(T^{*}\left(T^{k-1} Q\right)\right)$ e $\omega_{k-1} \in \Omega^{2}\left(T^{*}\left(T^{k-1} Q\right)\right)$ são as 1-forma e 2-forma canônicas de $T^{*}\left(T^{k-1} Q\right)$, respectivamente.

Pela expressão local de $\theta_{L}$ a expressão local de $\operatorname{Leg}_{L}$ fica

$$
\operatorname{Leg}_{L}\left(q_{(0)}^{A}, \ldots, q_{(2 k-1)}^{A}\right)=\left(q_{(0)}^{A}, \ldots, q_{(k-1)}^{A}, p_{A}^{(0)}, \ldots, p_{A}^{(k-1)}\right),
$$

onde

$$
p_{A}^{(s-1)}=\sum_{i=0}^{k-s}(-1)^{i} d_{T}^{i}\left(\frac{\partial L}{\partial q_{(s+i)}^{A}}\right)
$$

para $s=1, \ldots, k$. Note que com isso a forma $\theta_{L}$ fica escrita como

$$
\theta_{L}=\sum_{r=1}^{k} p_{A}^{(r-1)} d q_{(r-1)}^{A},
$$


e ao longo de uma curva em $T^{2 k-1} Q$ as coordenadas $p_{A}^{(s-1)}$ ficam

$$
p_{A}^{(s-1)}=\sum_{i=0}^{k-s}(-1)^{i} \frac{d^{i}}{d t^{i}}\left(\frac{\partial L}{\partial q_{(s+i)}^{A}}\right), \quad s=1, \ldots, k .
$$




\section{Referências Bibliográficas}

[ABR13] A. Agrachev, D. Barilari e L. Rizzi. Curvature: a variational approach. pre-print, 2013. arXiv:1306.5318. 1

[And] I. M. Anderson. The variational bicomplex. to appear. 62

[ÁPD09] J. C. Álvarez-Paiva e C. E. Durán. Geometric invariants of fanning curves. Advances in Applied Mathematics, 42:290-312, 2009. 1, 3, 4, 5, 6, 7, 37, 41, 42, 50

[Cim30] G. Cimmino. Autosoluzioni e autovalori nelle equazioni differenziali lineari ordinarie autoaggiunte di ordine superiore. Math. Z., 32:4-58, 1930. 12

[Cim39] G. Cimmino. Estensione dell'identita di picone alia più generale equazione differenziale lineare ordinaria autoaggiuntar. Atti Accad. Lincei Rend., 28:354-364, 1939. 12

[Cop71] W. A. Coppel. Disconjugacy. Springer-Verlag, 1971. 3, 12, 15, 16, 18, 26, 27

[Cra83] M. Crampin. Defining Euler-Lagrange fields in terms of almost tangent structure. Physics Letters, 95:466-468, 1983. 51, 54

[dAP10] C. R. de A. Peixoto. Invariantes de Curvas em Grassmannianas Divisiveis e Equações Diferenciais Ordinárias. Tese de Doutorado, Universidade Estadual de Campinas UNICAMP, Agosto 2010. 2, 7, 16

[DdAP14] C. E. Durán e C. R. de A. Peixoto. Geometry of fanning curves in divisible grassmannians. pre-print, 2014. arXiv:1412.3287. 4, 7, 30

[dLR85] M. de León e P. R. Rodrigues. Generalized Classical Mechanics and Field Theory. Elsevier Science Publisher, 1985. 4, 25

[dLR92] M. de Léon e P. R. Rodrigues. The inverse problem of Lagrangian dynamics for higherorder differential equations: a geometrical approach. Inverse Problems, 8(4):525-540, 1992. 51,61

[Dou41] J. Douglas. Solution of the inverse problem of the calculus of variations. Trans. Amer. Math. Soc., 50:71-128, 1941. 37

[Eas73] M. S. P. Eastham. The Picone identity for self-adjoint differential equations of even order. Mathematika, 20(2):197-200, 1973. 12

[Eas76] S. Easwaran. Quadratic functionals of nth order. Pacific Journal of Mathematics, 19(2):159-167, 1976. 3, 9, 11

[GF00] I. M. Gelfand e S. V. Fomin. Calculus of Variations. Dover PUblications, 2000. 4, 10, 12,51

[GMPT99] F. Giannoni, A. Masiello, P. Piccione e D. V. Tausk. A generalized index theorem for Morse-Sturm systems and applications to semi-riemannian geometry. pre-print, 1999. arXiv:math/9908056v1. 1 
[Hen82] M. Henneaux. Equations of motion, commutation relations and ambiguities in the Lagrangian formalism. Annals Phys., 140(1):45-64, 1982. 37

[JV14] M. A. Javaloyes e H. Vitório. Zermelo navigation in pseudo-Finsler metrics. pre-print, 2014. arXiv:1412.0465v1. 1

[KN69] S. Kobayashi e K. Nomizu. The Foundations of Differential Geometry. Wiley Intersc., 1969. 61

[Kra95] W. Kratz. An oscillation theorem for self-adjoint differential systems and the Rayleigh principle for quadratic funcionals. J. London Math. Soc., 51(2):401-416, 1995. 16

[Kre73] K. Kreith. Oscillation Theory. Springer, 1973. 12

[Lei70] W. Leighton. Quadratic functionals of second order. Transactions of The American Mathematical Society, 151:309-322, 1970. 12

[Mil73] J. Milnor. Morse Theory. Princeton University Press, 1973. 4

[MPT02] F. Mercuri, P. Piccione e D. V. Tausk. Stability of the conjugate index, degenerate conjugate points and the Maslov index in semi-riemannian geometry. Pacific Journal of Mathematics, 206(2):375-400, 2002. 1

[Pal63] R. Palais. Morse theory on Hilbert manifolds. Topology, 2:299-340, 1963. 4

[Pal68] R. Palais. Foundations of Global Non-linear Analysis. Benjamin and Co. New York, 1968. 4

[Pal69] R. Palais. The Morse lemma for Banach spaces. Bull. Amer. Math. Soc., 75:968-971, 1969. 4

[Pat99] G. P. Paternain. Geodesics Flows. Birkhauser, 1999. 1

[Pic10] M. Picone. Sui valori eccezionali di un parametro da cui dipende un'equazione differenziale lineare del secondo ordine. Ann. Scuola Norm. Sup. Pisa, 11:1-141, 1910. 12

[Rei80] W. T. Reid. Sturmian Theory for Ordinary Differential Equations. Springer, 1980. 12

[Rug07] R. O. Ruggiero. Dynamics and global geometry of manifolds without conjugate points. Sociedade Brasileira de Matemática, 12(1):1-181, 2007. 1

[SEB82] W. Sarlet, E. Engels e L. Y. Bahar. Time-dependent linear systems derivable from a variational principle. International Journal of Engineering Science, 20(1):55-66, 1982. 37,44 